\title{
Side-Arm Functionalized Silylene Copper(I) Complexes in Catalysis
}

Alexander N. Paesch, Anne-Kathrin Kreyenschmidt, Regine Herbst-Irmer, and Dietmar Stalke* 


\section{Supporting Information}

\section{Content:}

S1. Experimental Section (p.3-6)

S2. X-Ray Crystallographic Analysis (p.7-36)

S3. NMR Spectroscopic Analysis (p.37-53)

S4. References (p.54) 


\section{S1. Experimental Section}

General Procedures All reactions were carried out under an atmosphere of $\mathrm{N}_{2}$ and Ar by Schlenk techniques. ${ }^{[1]}$ All solvents were distilled from Na or $\mathrm{K}$ before used for synthesis. Starting materials were purchased commercially and used without further purification. MesNHPy and

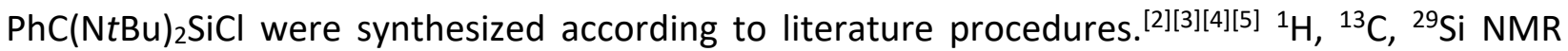
spectroscopic data were recorded on a Bruker Avance $401 \mathrm{MHz}$ and a Bruker Avance $300 \mathrm{MHz}$ spectrometer and referenced to the deuterated solvent (benzene- $d_{6}, d c m-d_{2}$, thf- $d_{8}$ ). Deuterated solvents were dried over activated molecular sieves $(3 \AA)$ and stored in an argon dry box. Elemental analyses (C, H, N) were carried out on a Vario EL3 at the Mikroanalytisches Labor, Institut für Anorganische Chemie, University of Göttingen. LIFDI-MS spectra were measured on a Jeol AccuTOF spectrometer.

General procedure for the preparation of 2. The copper precursor (1.00 eq.) was taken up in thf $(2 \mathrm{~mL})$ to form a white suspension and was subsequently cooled to $0{ }^{\circ} \mathrm{C}$. The chloro silylene $\mathrm{PhC}(\mathrm{NtBu})_{2} \mathrm{SiCl} 1$ (1.00 eq.) was separately dissolved in thf $(5 \mathrm{~mL})$ and slowly added under vigorous stirring. The reaction mixture was allowed to warm up to room temperature and stirred overnight. After filtration the yellow filtrate was concentrated and stored at $-35^{\circ} \mathrm{C}$ for $1 \mathrm{~d}$ to gain the target compounds in form of crystals suitable for X-ray analysis.

Preparation of 2a. Following the general procedure described above $\mathrm{CuCl}(168 \mathrm{mg}, 1.70 \mathrm{mmol})$ and $\mathrm{PhC}(\mathrm{NtBu})_{2} \mathrm{SiCl} 1(500 \mathrm{mg}, 1.70 \mathrm{mmol})$ were used and resulted in pale yellow crystals (448.8 mg, $0.285 \mathrm{mmol}, 67 \%$ ).

${ }^{1} \mathrm{H}-\mathrm{NMR}\left(300.13 \mathrm{MHz}, \mathrm{dcm}-\mathrm{d}_{2}, \mathrm{ppm}\right): 7.72-7.69$ (m, 1H, CPh), 7.60-7.49 (m, 4H, CPh), 1.26 (s, 18H, $\left.\mathrm{N}^{t} B u\right) .{ }^{13} \mathrm{C}\left\{{ }^{1} \mathrm{H}\right\}-\mathrm{NMR}\left(75.48 \mathrm{MHz}, \mathrm{dcm}-\mathrm{d}_{2}, \mathrm{ppm}\right): 172.11$ (CPh), 131.21 (CPh), 130.59 (CPh), 128.68 (CPh), 128.38 (CPh), $\left.128.07(\mathrm{CPh}), 54.33\left(\mathrm{NC} \mathrm{CH}_{3}\right)_{3}\right), 30.53\left(\mathrm{~N}^{t} \mathrm{Bu}\right) .{ }^{29} \mathrm{Si}\left\{{ }^{1} \mathrm{H}\right\}-\mathrm{NMR}(79.49 \mathrm{MHz}, \mathrm{dcm}-$ $\left.d_{2}, p p m\right):$ 27.73. Elemental analysis (\%) calcd for $\mathrm{C}_{60} \mathrm{H}_{92} \mathrm{~N}_{8} \mathrm{Si}_{4} \mathrm{Cl}_{8} \mathrm{Cu}_{4}: \mathrm{C}, 45.74 ; \mathrm{H}, 5.89 ; \mathrm{N}, 7.11$; found $C, 45.44 ; H, 5.65 ; N, 7.31$. 
Preparation of $\mathbf{2 b}$. Following the general procedure described above $\mathrm{CuBr}\left(\mathrm{SMe}_{2}\right)(348 \mathrm{mg}$, $1.70 \mathrm{mmol})$ and $\mathrm{PhC}(\mathrm{NtBu})_{2} \mathrm{SiCl} 1(500 \mathrm{mg}, 1.70 \mathrm{mmol})$ were used and resulted in yellowish crystals (550 mg, $0.302 \mathrm{mmol}, 71 \%)$.

${ }^{1} \mathrm{H}-\mathrm{NMR}(400.13 \mathrm{MHz}$, benzene-d6, ppm): $7.35(\mathrm{~d}, J=8.1 \mathrm{~Hz}, 1 \mathrm{H}, \mathrm{CPh}), 7.13-6.98(\mathrm{~m}, 4 \mathrm{H}, \mathrm{CPh})$, $1.21\left(\mathrm{~s}, 18 \mathrm{H}, \mathrm{N}^{t} B u\right) .{ }^{13} \mathrm{C}\left\{{ }^{1} \mathrm{H}\right\}-\mathrm{NMR}(100.62 \mathrm{MHz}$, benzene-d $6, \mathrm{ppm}): 171.52(\mathrm{CPh}), 131.08(\mathrm{CPh})$, 130.09 (CPh), 128.35 (CPh), 128.32 (CPh), 127.66 (CPh), $54.29\left(\mathrm{NC}(\mathrm{Me})_{3}\right), 30.75\left(\mathrm{~N}^{t} \mathrm{Bu}\right)$. ${ }^{29} \mathrm{Si}\left\{{ }^{1} \mathrm{H}\right\}-N M R(79.49 \mathrm{MHz}$, benzene-d, $\mathrm{ppm}):$ 26.09. MS (ESI[+], thf): $\mathrm{m} / \mathrm{z}=2144.78(<5)\left[\mathrm{M}-\mathrm{Br}^{-}\right.$ $\left.+2 \mathrm{SbF}_{6}\right]^{+}$. Elemental analysis (\%) calcd for $\mathrm{C}_{60} \mathrm{H}_{92} \mathrm{~N}_{8} \mathrm{Si}_{4} \mathrm{Br}_{4} \mathrm{Cl}_{4} \mathrm{Cu}_{4}\left(\mathrm{C}_{7} \mathrm{H}_{8}\right)$ : C, 43.60; $\mathrm{H}, 5.46 ; \mathrm{N}, 6.07$; found $\mathrm{C}, 43.00 ; \mathrm{H}, 5.40 ; \mathrm{N}, 5.96$.

Preparation of 2c. Following the general procedure described above Cul (323 mg, $1.70 \mathrm{mmol}$ ) and $\mathrm{PhC}(\mathrm{NtBu})_{2} \mathrm{SiCl} 1(500 \mathrm{mg}, 1.70 \mathrm{mmol})$ were used and resulted in yellow crystals $(454 \mathrm{mg}$, $0.234 \mathrm{mmol}, 55 \%)$.

${ }^{1} \mathrm{H}-\mathrm{NMR}\left(300.13 \mathrm{MHz}, \mathrm{dcm}-\mathrm{d}_{2}, \mathrm{ppm}\right):$ 7.66-7.48 (m, 5H, CPh), 1.30 (s, 18H, N $\left.{ }^{t} B u\right) .{ }^{13} \mathrm{C}\left\{{ }^{1} \mathrm{H}\right\}-\mathrm{NMR}$ (75.48 MHz, dcm-d, $\mathrm{ppm}$ ): 172.14 (CPh), 130.87 (CPh), 130.63 (CPh), 128.63 (CPh), 128.50 (CPh), $128.16(\mathrm{CPh}), 54.58\left(\mathrm{NC}\left(\mathrm{CH}_{3}\right)_{3}\right), 31.11\left(\mathrm{~N}^{t} \mathrm{Bu}\right) .{ }^{29} \mathrm{Si}\left\{{ }^{1} \mathrm{H}\right\}-\mathrm{NMR}\left(79.49 \mathrm{MHz}, \mathrm{dcm}-\mathrm{d}_{2}, \mathrm{ppm}\right): 22.62$. Elemental analysis (\%) calcd for $\mathrm{C}_{60} \mathrm{H}_{92} \mathrm{~N}_{8} \mathrm{Si}_{4} \mathrm{I}_{4} \mathrm{Cl}_{4} \mathrm{Cu}_{4}\left(\mathrm{C}_{7} \mathrm{H}_{8}\right): \mathrm{C}, 39.57 ; \mathrm{H}, 4.96 ; \mathrm{N}, 5.51$; found $\mathrm{C}$, $38.92 ; \mathrm{H}, 4.61 ; \mathrm{N}, 5.42$.

General procedure for the preparation of 3. The compound 2 (1.00 eq.) and the amine MesNHPy (4.00 eq.) were dissolved in a mixture of toluene $(3 \mathrm{~mL})$ and thf $(3 \mathrm{~mL})$ in a ratio of $1: 1$ and cooled down to $-30^{\circ} \mathrm{C}$. A separately prepared solution of LiHMDS (4.00 eq) in thf $(2 \mathrm{~mL})$ was added dropwise and subsequently allowed to warm up to ambient temperature under constant stirring for approximately $16 \mathrm{~h}$. The resulting suspension was concentrated until a yellow solid precipitated which was filtered off and dissolved in $\mathrm{dcm}$ in order to fully remove the $\mathrm{LiCl}$ salt in a further filtration step. The concentrated solution was stored at $-35^{\circ} \mathrm{C}$ for one day. The target compounds were isolated in form of yellow crystals suitable for X-ray analysis.

Preparation of 3a. Following the general procedure described above $2 \mathrm{a}(213 \mathrm{mg}, 0.135 \mathrm{mmol})$, MesNHPy (115 mg, $0.540 \mathrm{mmol}$ ) and LiHMDS (90.4 mg, $0.540 \mathrm{mmol})$ were used and resulted in yellow crystals (183 $\mathrm{mg}, 0.148 \mathrm{mmol}, 55 \%)$. 
${ }^{1} \mathrm{H}-\mathrm{NMR}\left(300.13 \mathrm{MHz}, \mathrm{dcm}-\mathrm{d}_{2}, \mathrm{ppm}\right): 8.39$ (br s, 1H, NPy), 7.61-7.42 (m, 5H, CPh), 7.45 (ddd, $J=$ $8.8 \mathrm{~Hz}, 6.2,1.9 \mathrm{~Hz}, 1 \mathrm{H}, \mathrm{NPy}), 7.06$ (s, 2H, NMes), 6.80 (t, J = 6.2 Hz, 1H, NPy), 6.01 (dt, J = 8.5, 1.0 $\mathrm{Hz}, 1 \mathrm{H}, \mathrm{NPy}), 2.39-2.35$ (m, 9H, MesCH3), 1.11 (br s, $\left.18 \mathrm{H}, \mathrm{N}^{t} B u\right) .{ }^{13} \mathrm{C}\left\{{ }^{1} \mathrm{H}\right\}-\mathrm{NMR}(75.48 \mathrm{MHz}, \mathrm{dcm}-$ $\mathrm{d}_{2}, \mathrm{ppm}$ ): 172.09 (CPh), 160.05 (NPy), 146.05 (NPy), 138.71 (NMes), 137.92 (NMes), 137.24 (NMes), 136.46 (NMes), 130.57 (NPy), 129.62 (CPh), 128.96 (NMes), 128.38 (CPh), 128.15 (CPh), 128.00 (CPh), 114.42 (NPy), 108.16 (NPy), $54.73\left(\mathrm{NC}\left(\mathrm{CH}_{3}\right)_{3}\right), 31.32\left(\mathrm{~N}^{t} \mathrm{Bu}\right), 20.66$ (MesMe), 19.38 (MesMe). MS (LIFDI[+], thf): $\mathrm{m} / \mathrm{z}=1117.2$ (5) [M-Cl], 919.4 (100) [M-Cu $\left.2 \mathrm{Cl}_{3}\right]^{+}$. Elemental analysis (\%) calcd for $\mathrm{C}_{58} \mathrm{H}_{76} \mathrm{~N}_{8} \mathrm{Si}_{2} \mathrm{Cl}_{3} \mathrm{Cu}_{3}\left(\mathrm{C}_{7} \mathrm{H}_{8}\right)_{2}: \mathrm{C}, 60.78 ; \mathrm{H}, 6.52 ; \mathrm{N}, 7.88$; found $\mathrm{C}, 61.38 ; \mathrm{H}, 6.33 ; \mathrm{N}, 7.70$.

Preparation of $\mathbf{3 b}$. Following the general procedure described above $\mathbf{2 b}(234.0 \mathrm{mg}, 0.134 \mathrm{mmol})$, MesNHPy (114 mg, $0.535 \mathrm{mmol}$ ) and LiHMDS (89.5 mg, $0.535 \mathrm{mmol})$ were used and resulted in yellow crystals (77.8 $\mathrm{mg}, 0.0567 \mathrm{mmol}, 42 \%)$.

${ }^{1} \mathrm{H}-\mathrm{NMR}\left(300.13 \mathrm{MHz}, \mathrm{dcm}-\mathrm{d}_{2}, \mathrm{ppm}\right):$ 8.27-8.17 (m, 1H, NPy), 7.66-7.49 (m, 3H, CPh), 7.45 (ddd, $J=8.8,7.1,1.9 \mathrm{~Hz}, 1 \mathrm{H}, \mathrm{NPy}), 7.42-7.35$ (m, 2H, CPh), 7.06 (s, 2H, NMes), 6.68 (ddd, J = 6.6, 5.3, $1.1 \mathrm{~Hz}, 1 \mathrm{H}, \mathrm{NPy}), 6.17(\mathrm{~d}, \mathrm{~J}=8.5 \mathrm{~Hz}, 1 \mathrm{H}, \mathrm{NPy}), 2.35$ (s, 3H, MesCH3), $2.31\left(\mathrm{~s}, 6 \mathrm{H}, \mathrm{Mes} C H_{3}\right), 1.05$ (s, $\left.18 \mathrm{H}, \mathrm{N}^{t} B u\right) .{ }^{13} \mathrm{C}\left\{{ }^{1} \mathrm{H}\right\}-\mathrm{NMR}\left(75.48 \mathrm{MHz}, \mathrm{dcm}-\mathrm{d}_{2}, \mathrm{ppm}\right): 170.81$ (CPh), 162.30 (NPy), 148.10 (NPy), 139.35 (NMes), 138.74 (NPy), 136.20 (NMes), 136.10 (NMes), 131.63 (CPh), 130.74 (CPh), 129.81

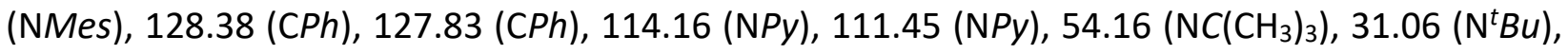
20.54 (MesMe), 19.70 (MesMe). MS (LIFDI[+], thf): m/z = 1435.0 (100) [M-Br+2thf] ${ }^{+}, 1291.2$ (5) $[\mathrm{M}-\mathrm{Br}]^{+}$. Elemental analysis (\%) calcd for $\mathrm{C}_{58} \mathrm{H}_{76} \mathrm{~N}_{8} \mathrm{Si}_{2} \mathrm{Br}_{3} \mathrm{Cu}_{3}\left(\mathrm{C}_{7} \mathrm{H}_{8}\right)_{2}: \mathrm{C}, 55.57 ; \mathrm{H}, 5.96 ; \mathrm{N}, 7.20$; found C, 58.26; H, 5.71; N, 7.19.

Preparation of 3c. Following the general procedure described above 2c (334 mg, $0.172 \mathrm{mmol}$ ), MesNHPy (146 mg, $0.688 \mathrm{mmol}$ ) and LiHMDS (115 mg, $0.688 \mathrm{mmol}$ ) were used and resulted in yellow crystals (159 mg, $0.105 \mathrm{mmol}, 31 \%)$.

${ }^{1} \mathrm{H}-\mathrm{NMR}\left(300.13 \mathrm{MHz}, \mathrm{dcm}-\mathrm{d}_{2}, \mathrm{ppm}\right): 8.72$ (br s, 1H, NPy), 7.59-7.40 (m, 5H, CPh), 7.48-7.42 (m, 1H, NPy), 7.08 (s, 2H, NMes), 6.79 (ddd, J = 6.8, 5.4, 1.1 Hz, 1H, NPy), 6.00 (d, J = 8.5 Hz, 1H, NPy), 2.41-2.37 (m, 9H, MesCH $\left.\mathrm{CH}_{3}\right), 1.04\left(\mathrm{~s}, 18 \mathrm{H}, \mathrm{N}^{t} B u\right) .{ }^{13} \mathrm{C}\left\{{ }^{1} \mathrm{H}\right\}-\mathrm{NMR}\left(75.48 \mathrm{MHz}, \mathrm{dcm}-\mathrm{d}_{2}, \mathrm{ppm}\right): 172.68$ (CPh), 161.13 (NPy), 148.57 (NPy), 139.93 (NMes), 138.96 (NPy), 137.93 (NMes), 137.34 (NMes), 131.68 (CPh), 130.47 (CPh), 129.82 (NMes), 128.96 (CPh), 128.15 (CPh), 114.46 (NPy), 110.31 
(NPy), 54.68( $\left.\mathrm{NC}\left(\mathrm{CH}_{3}\right)_{3}\right), 31.28\left(\mathrm{~N}^{t} \mathrm{Bu}\right), 20.60$ (MesMe), 20.12 (MesMe). MS (LIFDI[+], thf): $\mathrm{m} / \mathrm{z}=$ $1385.3(10)[\mathrm{M}-1]^{+}, 1003.6(<5)\left[\mathrm{M}-\mathrm{Cu}_{2} \mathrm{I}_{3}\right]^{+}$. Elemental analysis (\%) calcd for $\mathrm{C}_{58} \mathrm{H}_{76} \mathrm{~N}_{8} \mathrm{Si}_{2} \mathrm{I}_{3} \mathrm{Cu}_{3}\left(\mathrm{C}_{7} \mathrm{H}_{8}\right)$ : C, 48.64; H, 5.28; N, 6. 98; found C, 53.53; H, 5.21; N, 7.11. 


\section{S2. X-Ray Crystallographic Analysis}

TABLE 1. Crystal data for $2 \mathrm{a}-\mathrm{c}$ and $3 \mathrm{a}-\mathrm{c}$ at $100(2) \mathrm{K}$.

\begin{tabular}{|c|c|c|c|c|c|c|}
\hline & $2 \mathbf{a}$ & $2 \mathbf{b}$ & $2 \mathrm{c}$ & $3 \mathbf{a}$ & $3 \mathbf{b}$ & $3 c$ \\
\hline Empirical & $\mathrm{C}_{76} \mathrm{H}_{124} \mathrm{~N}_{8} \mathrm{Si}_{4}$ & $\mathrm{C}_{81} \mathrm{H}_{116} \mathrm{~N}_{8} \mathrm{Si}_{4}$ & $\mathrm{C}_{76} \mathrm{H}_{124} \mathrm{~N}_{8} \mathrm{Si}_{4}$ & $\mathrm{C}_{79} \mathrm{H}_{100} \mathrm{~N}_{8} \mathrm{Si}_{2}$ & $\mathrm{C}_{65} \mathrm{H}_{84} \mathrm{~N}_{8} \mathrm{Si}_{2}$ & $\mathrm{C}_{79} \mathrm{H}_{100} \mathrm{~N}_{8} \mathrm{Si}_{2}$ \\
\hline formula & $\mathrm{Cl}_{8} \mathrm{Cu}_{4} \mathrm{O}_{4}$ & $\mathrm{Cl}_{4.25} \mathrm{Br}_{3.75} \mathrm{Cu}_{4}$ & $\mathrm{Cl}_{4.57} \mathrm{I}_{3.43} \mathrm{Cu}_{4} \mathrm{O}_{4}$ & $\mathrm{Cl}_{3} \mathrm{Cu}_{3}$ & $\mathrm{Cl}_{0.43} \mathrm{Br}_{2.57} \mathrm{Cu}_{3}$ & $\mathrm{Cl}_{0.92} \mathrm{I}_{2.08} \mathrm{Cu}_{3}$ \\
\hline$M_{r}[\mathrm{~g} / \mathrm{mol}]$ & 1863.94 & 2018.44 & 2177.32 & 1514.81 & 1444.76 & 1704.96 \\
\hline rmm & $0.331 \times 0.314 \mathrm{x}$ & $0.204 \times 0.184 x$ & $0.301 \times 0.201 x$ & $0.348 \times 0.150 \mathrm{x}$ & $0.205 \times 0.136 \mathrm{x}$ & $0.284 \times 0.278 x$ \\
\hline [mm] & 0.080 & 0.124 & 0.147 & 0.090 & 0.088 & 0.174 \\
\hline Cryst. syst. & monoclinic & monoclinic & monoclinic & triclinic & triclinic & triclinic \\
\hline Space group & $\mathrm{C} 2 / \mathrm{c}$ & $\mathrm{C} 2 / \mathrm{c}$ & $\mathrm{C} 2 / \mathrm{c}$ & P-1 & $\mathrm{P}-1$ & P-1 \\
\hline$a[\AA]$ & $19.064(2)$ & $20.130(3)$ & $19.751(2)$ & $10.376(2)$ & $10.460(2)$ & $10.498(3)$ \\
\hline$b[\AA]$ & $18.785(2)$ & $18.444(2)$ & $18.807(2)$ & $18.154(2)$ & $18.205(4)$ & $18.205(2)$ \\
\hline$c[\AA]$ & $25.746(3)$ & $26.594(3)$ & $25.572(3)$ & $20.423(3)$ & $20.522(4)$ & $20.437(2)$ \\
\hline $\boldsymbol{\alpha}\left[^{\circ}\right]$ & 90 & 90 & 90 & $89.56(2)$ & $88.29(3)$ & $87.32(2)$ \\
\hline $\boldsymbol{\beta}\left[^{\circ}\right]$ & $103.96(2)$ & $109.17(3)$ & $103.53(2)$ & $77.14(2)$ & $77.36(2)$ & $77.16(2)$ \\
\hline$\gamma\left[^{\circ}\right]$ & 90 & 90 & 90 & $89.75(3)$ & $89.01(3)$ & $88.91(3)$ \\
\hline Volume $\left[\AA^{3}\right]$ & $8947.8(19)$ & $9326(3)$ & $9235.3(19)$ & $3750.4(10)$ & $3811.2(14)$ & $3803.9(13)$ \\
\hline $\mathbf{Z}$ & 4 & 4 & 4 & 2 & 2 & 2 \\
\hline$\mu\left[\mathbf{m m}^{-1}\right]$ & $1.280\left(\mathrm{Mo} \mathrm{K}_{\alpha}\right)$ & $2.722\left(\mathrm{Mo} \mathrm{K}_{\alpha}\right)$ & $1.206\left(\mathrm{Ag} \mathrm{K}_{\alpha}\right)$ & $1.030\left(\mathrm{Mo} \mathrm{K}_{\alpha}\right)$ & $2.262\left(\mathrm{Mo} \mathrm{K}_{\alpha}\right)$ & $\left.1.489(\mathrm{Ag} \mathrm{K})_{\alpha}\right)$ \\
\hline $\boldsymbol{\Theta}_{\max }\left[{ }^{\circ}\right]$ & 25.420 & 26.363 & 19.823 & 25.455 & 25.455 & 19.827 \\
\hline Refl. meas./indep. & $109474 / 8240$ & $136628 / 9521$ & $130581 / 8542$ & $99149 / 13774$ & $213816 / 14039$ & $99962 / 14073$ \\
\hline $\boldsymbol{R}_{\text {int }}$ & 0.0300 & 0.0334 & 0.0391 & 0.0401 & 0.0715 & 0.0376 \\
\hline Parameter & 572 & 527 & 651 & 941 & 754 & 1008 \\
\hline Restraints & 466 & 230 & 897 & 913 & 0 & 1593 \\
\hline$R_{1}[\mathrm{I}>2 \sigma(\mathrm{I})]$ & 0.0377 & 0.0262 & 0.0349 & 0.0509 & 0.0450 & 0.0270 \\
\hline$w R_{2}$ (all data) & 0.1005 & 0.0677 & 0.0893 & 0.1432 & 0.1236 & 0.0748 \\
\hline res. dens. peaks $\left[\mathrm{e}^{-3}\right]$ & 0.474 to -0.387 & 0.933 to -0.400 & 1.061 to -0.763 & 1.095 to -1.461 & 1.274 to -0.670 & 1.187 to -0.605 \\
\hline
\end{tabular}




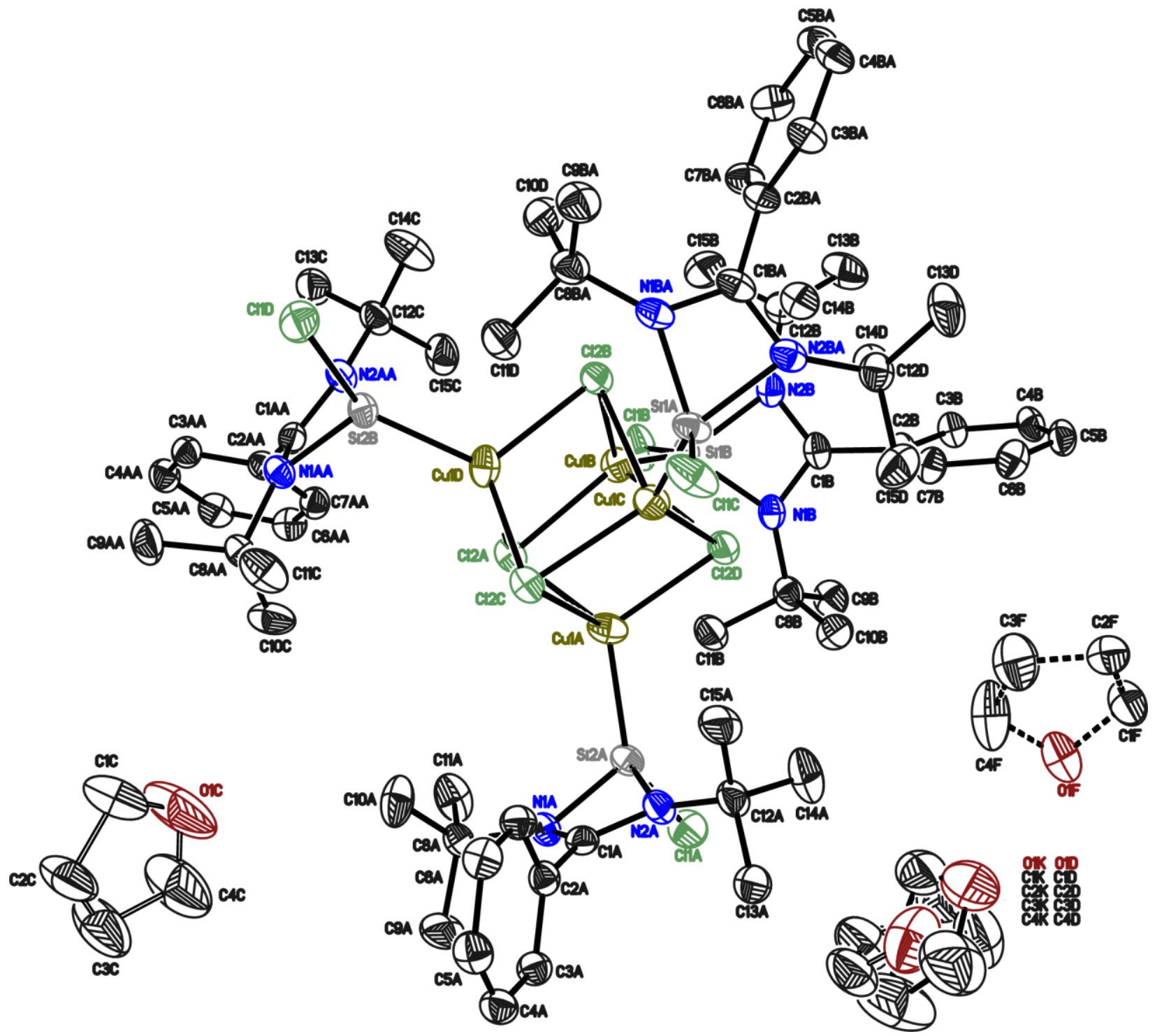

The carbon and oxygen atoms of the THF molecule (O1K-C4K/O1D-C4D) are disordered about two positions, while the THF molecule $\mathrm{O} 1 \mathrm{C}-\mathrm{C} 4 \mathrm{C}$ is disordered about a two-fold axis and the THF molecule O1F-C4F about an inversion center. They are refined with distance restraints and restraints for the anisotropic displacement parameters. The occupancy of the minor position refines to $0.443(13)$. 
Bond lengths $[\AA \AA]$ and angles $\left[^{\circ}\right]$ for $2 \mathrm{a}$ :

\begin{tabular}{|c|c|c|c|}
\hline $\mathrm{Si}(1 \mathrm{~B})-\mathrm{N}(1 \mathrm{~B})$ & $1.820(2)$ & $\mathrm{N}(2 \mathrm{~A})-\mathrm{Si}(2 \mathrm{~A})-\mathrm{Cl}(1 \mathrm{~A})$ & $103.94(9)$ \\
\hline $\mathrm{Si}(1 \mathrm{~B})-\mathrm{N}(2 \mathrm{~B})$ & $1.836(3)$ & $N(1 A)-S i(2 A)-C u(1 A)$ & $120.73(8)$ \\
\hline $\mathrm{Si}(1 \mathrm{~B})-\mathrm{Cl}(1 \mathrm{~B})$ & $2.1064(12)$ & $N(2 A)-S i(2 A)-C u(1 A)$ & $118.62(9)$ \\
\hline $\mathrm{Si}(1 \mathrm{~B})-\mathrm{Cu}(1 \mathrm{~B})$ & $2.1889(8)$ & $\mathrm{Cl}(1 \mathrm{~A})-\mathrm{Si}(2 \mathrm{~A})-\mathrm{Cu}(1 \mathrm{~A})$ & $125.59(5)$ \\
\hline $\mathrm{Si}(1 \mathrm{~B})-\mathrm{C}(1 \mathrm{~B})$ & $2.273(3)$ & $N(1 A)-S i(2 A)-C(1 A)$ & $36.01(10)$ \\
\hline $\mathrm{Cu}(1 \mathrm{~B})-\mathrm{Cl}(2 \mathrm{~B})$ & $2.3097(8)$ & $N(2 A)-S i(2 A)-C(1 A)$ & $35.98(10)$ \\
\hline $\mathrm{Cu}(1 \mathrm{~B})-\mathrm{Cl}(2 \mathrm{~A})$ & $2.3555(8)$ & $C l(1 A)-S i(2 A)-C(1 A)$ & $111.91(8)$ \\
\hline $\mathrm{Cu}(1 \mathrm{~B})-\mathrm{Cu}(1 \mathrm{~A})$ & $2.9740(6)$ & $\mathrm{Cu}(1 \mathrm{~A})-\mathrm{Si}(2 \mathrm{~A})-\mathrm{C}(1 \mathrm{~A})$ & $122.48(8)$ \\
\hline$C(1 B)-N(2 B)$ & $1.335(4)$ & $\mathrm{Cu}(1 \mathrm{~A})-\mathrm{Cl}(2 \mathrm{~A})-\mathrm{Cu}(1 \mathrm{~B})$ & $79.01(3)$ \\
\hline$C(1 B)-N(1 B)$ & $1.339(4)$ & $\mathrm{Cu}(1 \mathrm{~A})-\mathrm{Cl}(2 \mathrm{~A})-\mathrm{Cu}(1 \mathrm{~A}) \# 1$ & $87.38(3)$ \\
\hline$C(1 B)-C(2 B)$ & $1.488(4)$ & $\mathrm{Cu}(1 \mathrm{~B})-\mathrm{Cl}(2 \mathrm{~A})-\mathrm{Cu}(1 \mathrm{~A}) \# 1$ & $76.95(2)$ \\
\hline$N(1 B)-C(8 B)$ & $1.479(4)$ & $C(4 A)-C(3 A)-C(2 A)$ & $119.6(3)$ \\
\hline $\operatorname{Si}(2 A)-N(1 A)$ & $1.828(2)$ & $\mathrm{Cu}(1 \mathrm{~B})-\mathrm{Cl}(2 \mathrm{~B})-\mathrm{Cu}(\mathrm{AA}) \# 1$ & $86.01(3)$ \\
\hline $\operatorname{Si}(2 A)-N(2 A)$ & $1.835(3)$ & $C(7 B)-C(2 B)-C(3 B)$ & $119.9(3)$ \\
\hline $\mathrm{Si}(2 \mathrm{~A})-\mathrm{Cl}(1 \mathrm{~A})$ & $2.1110(11)$ & $C(7 B)-C(2 B)-C(1 B)$ & $118.9(2)$ \\
\hline $\mathrm{Si}(2 \mathrm{~A})-\mathrm{Cu}(1 \mathrm{~A})$ & $2.1930(9)$ & $C(3 B)-C(2 B)-C(1 B)$ & $121.1(2)$ \\
\hline $\operatorname{Si}(2 A)-C(1 A)$ & $2.279(3)$ & $C(1 B)-N(2 B)-C(12 B)$ & $131.3(2)$ \\
\hline $\mathrm{Cl}(2 \mathrm{~A})-\mathrm{Cu}(1 \mathrm{~A})$ & $2.3194(9)$ & $C(1 B)-N(2 B)-S i(1 B)$ & $90.13(18)$ \\
\hline $\mathrm{Cl}(2 \mathrm{~A})-\mathrm{Cu}(1 \mathrm{~A}) \# 1$ & $2.7497(8)$ & $\mathrm{C}(12 \mathrm{~B})-\mathrm{N}(2 \mathrm{~B})-\mathrm{Si}(1 \mathrm{~B})$ & 130.81(19) \\
\hline$C(3 A)-C(4 A)$ & $1.386(4)$ & $C(5 A)-C(4 A)-C(3 A)$ & $120.3(3)$ \\
\hline$C(3 A)-C(2 A)$ & $1.392(4)$ & $C(4 B)-C(3 B)-C(2 B)$ & $119.6(3)$ \\
\hline $\mathrm{Cl}(2 \mathrm{~B})-\mathrm{Cu}(1 \mathrm{~A}) \# 1$ & $2.3688(8)$ & $C(4 A)-C(5 A)-C(6 A)$ & $120.3(3)$ \\
\hline$C(2 B)-C(7 B)$ & $1.393(4)$ & $C(3 B)-C(4 B)-C(5 B)$ & $120.3(3)$ \\
\hline$C(2 B)-C(3 B)$ & $1.397(4)$ & $C(7 A)-C(6 A)-C(5 A)$ & $120.1(3)$ \\
\hline $\mathrm{N}(2 \mathrm{~B})-\mathrm{C}(12 \mathrm{~B})$ & $1.485(4)$ & $C(6 B)-C(5 B)-C(4 B)$ & $120.0(3)$ \\
\hline$C(4 A)-C(5 A)$ & $1.383(5)$ & $C(6 A)-C(7 A)-C(2 A)$ & $119.5(3)$ \\
\hline$C(3 B)-C(4 B)$ & $1.386(4)$ & $C(7 B)-C(6 B)-C(5 B)$ & $120.3(3)$ \\
\hline$C(5 A)-C(6 A)$ & $1.387(4)$ & $N(1 A)-C(8 A)-C(10 A)$ & $111.4(2)$ \\
\hline$C(4 B)-C(5 B)$ & $1.387(4)$ & $N(1 A)-C(8 A)-C(9 A)$ & $109.0(2)$ \\
\hline$C(6 A)-C(7 A)$ & $1.385(4)$ & $C(10 A)-C(8 A)-C(9 A)$ & $111.8(3)$ \\
\hline$C(5 B)-C(6 B)$ & $1.386(4)$ & $N(1 A)-C(8 A)-C(11 A)$ & $106.3(2)$ \\
\hline$C(7 A)-C(2 A)$ & $1.399(4)$ & $C(10 A)-C(8 A)-C(11 A)$ & $109.2(3)$ \\
\hline$C(6 B)-C(7 B)$ & $1.386(4)$ & $C(9 A)-C(8 A)-C(11 A)$ & $108.9(3)$ \\
\hline$C(8 A)-N(1 A)$ & $1.487(4)$ & $C(6 B)-C(7 B)-C(2 B)$ & $119.8(3)$ \\
\hline$C(8 A)-C(10 A)$ & $1.517(4)$ & $N(1 B)-C(8 B)-C(10 B)$ & $112.2(3)$ \\
\hline$C(8 A)-C(9 A)$ & $1.518(4)$ & $N(1 B)-C(8 B)-C(11 B)$ & $105.9(2)$ \\
\hline$C(8 A)-C(11 A)$ & $1.522(4)$ & $C(10 B)-C(8 B)-C(11 B)$ & $108.7(3)$ \\
\hline$C(8 B)-C(10 B)$ & $1.525(4)$ & $N(1 B)-C(8 B)-C(9 B)$ & $108.7(2)$ \\
\hline$C(8 B)-C(11 B)$ & $1.528(4)$ & $C(10 B)-C(8 B)-C(9 B)$ & $110.5(3)$ \\
\hline$C(8 B)-C(9 B)$ & $1.531(4)$ & $C(11 B)-C(8 B)-C(9 B)$ & $110.7(3)$ \\
\hline$C(12 A)-N(2 A)$ & $1.486(4)$ & $N(2 A)-C(12 A)-C(13 A)$ & $110.9(2)$ \\
\hline
\end{tabular}




\begin{tabular}{|c|c|c|c|}
\hline$C(12 A)-C(13 A)$ & $1.520(4)$ & $N(2 A)-C(12 A)-C(15 A)$ & $111.3(3)$ \\
\hline$C(12 A)-C(15 A)$ & $1.523(5)$ & $C(13 A)-C(12 A)-C(15 A)$ & $110.7(3)$ \\
\hline$C(12 A)-C(14 A)$ & $1.529(5)$ & $N(2 A)-C(12 A)-C(14 A)$ & $105.0(3)$ \\
\hline$C(12 B)-C(15 B)$ & $1.524(5)$ & $C(13 A)-C(12 A)-C(14 A)$ & $110.0(3)$ \\
\hline$C(12 B)-C(13 B)$ & $1.526(4)$ & $C(15 A)-C(12 A)-C(14 A)$ & $108.8(3)$ \\
\hline$C(12 B)-C(14 B)$ & $1.526(4)$ & $N(2 B)-C(12 B)-C(15 B)$ & $104.8(3)$ \\
\hline$C(2 A)-C(1 A)$ & $1.481(4)$ & $N(2 B)-C(12 B)-C(13 B)$ & $111.1(3)$ \\
\hline$N(2 A)-C(1 A)$ & $1.339(4)$ & $C(15 B)-C(12 B)-C(13 B)$ & $109.7(3)$ \\
\hline$C(1 A)-N(1 A)$ & $1.340(4)$ & $N(2 B)-C(12 B)-C(14 B)$ & $110.5(2)$ \\
\hline$O(1 C)-C(1 C)$ & $1.367(12)$ & $C(15 B)-C(12 B)-C(14 B)$ & $109.2(3)$ \\
\hline $\mathrm{O}(1 \mathrm{C})-\mathrm{C}(4 \mathrm{C})$ & $1.367(12)$ & $C(13 B)-C(12 B)-C(14 B)$ & $111.4(3)$ \\
\hline$C(1 C)-C(2 C)$ & $1.485(11)$ & $C(3 A)-C(2 A)-C(7 A)$ & $120.2(3)$ \\
\hline$C(2 C)-C(3 C)$ & $1.469(13)$ & $C(3 A)-C(2 A)-C(1 A)$ & $120.8(3)$ \\
\hline$C(3 C)-C(4 C)$ & $1.460(13)$ & $C(7 A)-C(2 A)-C(1 A)$ & $119.0(3)$ \\
\hline $\mathrm{O}(1 \mathrm{~K})-\mathrm{C}(4 \mathrm{~K})$ & $1.376(11)$ & $C(1 A)-N(2 A)-C(12 A)$ & $132.2(3)$ \\
\hline$O(1 K)-C(1 K)$ & $1.418(12)$ & $C(1 A)-N(2 A)-S i(2 A)$ & $90.41(18)$ \\
\hline$C(1 K)-C(2 K)$ & $1.492(12)$ & $C(12 A)-N(2 A)-S i(2 A)$ & $136.1(2)$ \\
\hline$C(2 K)-C(3 K)$ & $1.450(14)$ & $\mathrm{Si}(2 \mathrm{~A})-\mathrm{Cu}(1 \mathrm{~A})-\mathrm{Cl}(2 \mathrm{~A})$ & $131.85(3)$ \\
\hline$C(3 K)-C(4 K)$ & $1.514(13)$ & $\mathrm{Si}(2 \mathrm{~A})-\mathrm{Cu}(1 \mathrm{~A})-\mathrm{Cl}(2 \mathrm{~B}) \# 1$ & $114.34(3)$ \\
\hline$O(1 D)-C(4 D)$ & $1.370(12)$ & $\mathrm{Cl}(2 \mathrm{~A})-\mathrm{Cu}(1 \mathrm{~A})-\mathrm{Cl}(2 \mathrm{~B}) \# 1$ & $111.93(3)$ \\
\hline$O(1 D)-C(1 D)$ & $1.427(12)$ & $\mathrm{Si}(2 \mathrm{~A})-\mathrm{Cu}(1 \mathrm{~A})-\mathrm{Cl}(2 \mathrm{~A}) \# 1$ & $104.12(3)$ \\
\hline$C(1 D)-C(2 D)$ & $1.512(13)$ & $\mathrm{Cl}(2 \mathrm{~A})-\mathrm{Cu}(1 \mathrm{~A})-\mathrm{Cl}(2 \mathrm{~A}) \# 1$ & $86.65(3)$ \\
\hline$C(2 D)-C(3 D)$ & $1.488(15)$ & $\mathrm{Cl}(2 \mathrm{~B}) \# 1-\mathrm{Cu}(1 \mathrm{~A})-\mathrm{Cl}(2 \mathrm{~A}) \# 1$ & $92.03(3)$ \\
\hline$C(3 D)-C(4 D)$ & $1.484(13)$ & $\mathrm{Si}(2 \mathrm{~A})-\mathrm{Cu}(1 \mathrm{~A})-\mathrm{Cu}(1 \mathrm{~B})$ & $159.57(3)$ \\
\hline $\mathrm{O}(1 \mathrm{~F})-\mathrm{C}(4 \mathrm{~F})$ & $1.402(10)$ & $\mathrm{Cl}(2 \mathrm{~A})-\mathrm{Cu}(1 \mathrm{~A})-\mathrm{Cu}(1 \mathrm{~B})$ & $51.03(2)$ \\
\hline$O(1 F)-C(1 F)$ & $1.406(8)$ & $\mathrm{Cl}(2 \mathrm{~B}) \# 1-\mathrm{Cu}(1 \mathrm{~A})-\mathrm{Cu}(1 \mathrm{~B})$ & $61.59(2)$ \\
\hline$C(1 F)-C(2 F)$ & $1.489(10)$ & $\mathrm{Cl}(2 \mathrm{~A}) \# 1-\mathrm{Cu}(1 \mathrm{~A})-\mathrm{Cu}(1 \mathrm{~B})$ & $96.14(2)$ \\
\hline$C(2 F)-C(3 F)$ & $1.474(11)$ & $N(2 A)-C(1 A)-N(1 A)$ & $105.8(2)$ \\
\hline$C(3 F)-C(4 F)$ & $1.499(11)$ & $N(2 A)-C(1 A)-C(2 A)$ & $126.4(3)$ \\
\hline$N(1 B)-S i(1 B)-N(2 B)$ & $71.43(11)$ & $N(1 A)-C(1 A)-C(2 A)$ & $127.7(3)$ \\
\hline $\mathrm{N}(1 \mathrm{~B})-\mathrm{Si}(1 \mathrm{~B})-\mathrm{Cl}(1 \mathrm{~B})$ & 104.33(9) & $N(2 A)-C(1 A)-S i(2 A)$ & $53.61(14)$ \\
\hline $\mathrm{N}(2 \mathrm{~B})-\mathrm{Si}(1 \mathrm{~B})-\mathrm{Cl}(1 \mathrm{~B})$ & $103.61(8)$ & $N(1 A)-C(1 A)-S i(2 A)$ & $53.32(14)$ \\
\hline $\mathrm{N}(1 \mathrm{~B})-\mathrm{Si}(1 \mathrm{~B})-\mathrm{Cu}(1 \mathrm{~B})$ & $118.64(8)$ & $C(2 A)-C(1 A)-S i(2 A)$ & $168.0(2)$ \\
\hline $\mathrm{N}(2 \mathrm{~B})-\mathrm{Si}(1 \mathrm{~B})-\mathrm{Cu}(1 \mathrm{~B})$ & $121.25(9)$ & $C(1 A)-N(1 A)-C(8 A)$ & $131.0(2)$ \\
\hline $\mathrm{Cl}(1 \mathrm{~B})-\mathrm{Si}(1 \mathrm{~B})-\mathrm{Cu}(1 \mathrm{~B})$ & $124.75(4)$ & $C(1 A)-N(1 A)-S i(2 A)$ & $90.66(18)$ \\
\hline $\mathrm{N}(1 \mathrm{~B})-\mathrm{Si}(1 \mathrm{~B})-\mathrm{C}(1 \mathrm{~B})$ & $36.09(11)$ & $C(8 A)-N(1 A)-S i(2 A)$ & 133.60(19) \\
\hline$N(2 B)-S i(1 B)-C(1 B)$ & $35.98(11)$ & $C(1 C)-O(1 C)-C(4 C)$ & $112.3(7)$ \\
\hline $\mathrm{Cl}(1 \mathrm{~B})-\mathrm{Si}(1 \mathrm{~B})-\mathrm{C}(1 \mathrm{~B})$ & $112.43(8)$ & $\mathrm{O}(1 \mathrm{C})-\mathrm{C}(1 \mathrm{C})-\mathrm{C}(2 \mathrm{C})$ & 107.3(9) \\
\hline $\mathrm{Cu}(1 \mathrm{~B})-\mathrm{Si}(1 \mathrm{~B})-\mathrm{C}(1 \mathrm{~B})$ & $122.80(8)$ & $C(3 C)-C(2 C)-C(1 C)$ & $104.2(10)$ \\
\hline $\mathrm{Si}(1 \mathrm{~B})-\mathrm{Cu}(1 \mathrm{~B})-\mathrm{Cl}(2 \mathrm{~B})$ & $132.14(3)$ & $C(4 C)-C(3 C)-C(2 C)$ & $105.7(10)$ \\
\hline $\mathrm{Si}(1 \mathrm{~B})-\mathrm{Cu}(1 \mathrm{~B})-\mathrm{Cl}(2 \mathrm{~A})$ & $120.76(3)$ & $\mathrm{O}(1 \mathrm{C})-\mathrm{C}(4 \mathrm{C})-\mathrm{C}(3 \mathrm{C})$ & $107.3(10)$ \\
\hline $\mathrm{Cl}(2 \mathrm{~B})-\mathrm{Cu}(1 \mathrm{~B})-\mathrm{Cl}(2 \mathrm{~A})$ & $104.64(3)$ & $\mathrm{C}(4 \mathrm{~K})-\mathrm{O}(1 \mathrm{~K})-\mathrm{C}(1 \mathrm{~K})$ & $109.5(8)$ \\
\hline $\mathrm{Si}(1 \mathrm{~B})-\mathrm{Cu}(1 \mathrm{~B})-\mathrm{Cu}(\mathrm{AA})$ & $117.23(3)$ & $\mathrm{O}(1 \mathrm{~K})-\mathrm{C}(1 \mathrm{~K})-\mathrm{C}(2 \mathrm{~K})$ & 106.4(9) \\
\hline $\mathrm{Cl}(2 \mathrm{~B})-\mathrm{Cu}(1 \mathrm{~B})-\mathrm{Cu}(1 \mathrm{~A})$ & $102.74(3)$ & $C(3 K)-C(2 K)-C(1 K)$ & $107.8(10)$ \\
\hline
\end{tabular}




\begin{tabular}{|l|l|l|l|}
\hline $\mathrm{Cl}(2 \mathrm{~A})-\mathrm{Cu}(1 \mathrm{~B})-\mathrm{Cu}(1 \mathrm{~A})$ & $49.96(2)$ & $\mathrm{C}(2 \mathrm{~K})-\mathrm{C}(3 \mathrm{~K})-\mathrm{C}(4 \mathrm{~K})$ & $102.4(10)$ \\
\hline $\mathrm{N}(2 \mathrm{~B})-\mathrm{C}(1 \mathrm{~B})-\mathrm{N}(1 \mathrm{~B})$ & $105.9(2)$ & $\mathrm{O}(1 \mathrm{~K})-\mathrm{C}(4 \mathrm{~K})-\mathrm{C}(3 \mathrm{~K})$ & $108.2(9)$ \\
\hline $\mathrm{N}(2 \mathrm{~B})-\mathrm{C}(1 \mathrm{~B})-\mathrm{C}(2 \mathrm{~B})$ & $126.3(3)$ & $\mathrm{C}(4 \mathrm{D})-\mathrm{O}(1 \mathrm{D})-\mathrm{C}(1 \mathrm{D})$ & $102.5(10)$ \\
\hline $\mathrm{N}(1 \mathrm{~B})-\mathrm{C}(1 \mathrm{~B})-\mathrm{C}(2 \mathrm{~B})$ & $127.7(3)$ & $\mathrm{O}(1 \mathrm{D})-\mathrm{C}(1 \mathrm{D})-\mathrm{C}(2 \mathrm{D})$ & $103.3(10)$ \\
\hline $\mathrm{N}(2 \mathrm{~B})-\mathrm{C}(1 \mathrm{~B})-\mathrm{Si}(1 \mathrm{~B})$ & $53.89(14)$ & $\mathrm{C}(3 \mathrm{D})-\mathrm{C}(2 \mathrm{D})-\mathrm{C}(1 \mathrm{D})$ & $101.1(10)$ \\
\hline $\mathrm{N}(1 \mathrm{~B})-\mathrm{C}(1 \mathrm{~B})-\mathrm{Si}(1 \mathrm{~B})$ & $53.19(14)$ & $\mathrm{C}(4 \mathrm{D})-\mathrm{C}(3 \mathrm{D})-\mathrm{C}(2 \mathrm{D})$ & $103.7(9)$ \\
\hline $\mathrm{C}(2 \mathrm{~B})-\mathrm{C}(1 \mathrm{~B})-\mathrm{Si}(1 \mathrm{~B})$ & $167.04(19)$ & $\mathrm{O}(1 \mathrm{D})-\mathrm{C}(4 \mathrm{D})-\mathrm{C}(3 \mathrm{D})$ & $109.3(9)$ \\
\hline $\mathrm{C}(1 \mathrm{~B})-\mathrm{N}(1 \mathrm{~B})-\mathrm{C}(8 \mathrm{~B})$ & $132.7(2)$ & $\mathrm{C}(4 \mathrm{~F})-\mathrm{O}(1 \mathrm{~F})-\mathrm{C}(1 \mathrm{~F})$ & $105.5(7)$ \\
\hline $\mathrm{C}(1 \mathrm{~B})-\mathrm{N}(1 \mathrm{~B})-\mathrm{Si}(1 \mathrm{~B})$ & $90.72(18)$ & $\mathrm{O}(1 \mathrm{~F})-\mathrm{C}(1 \mathrm{~F})-\mathrm{C}(2 \mathrm{~F})$ & $105.9(6)$ \\
\hline $\mathrm{C}(8 \mathrm{~B})-\mathrm{N}(1 \mathrm{~B})-\mathrm{Si}(1 \mathrm{~B})$ & $136.57(19)$ & $\mathrm{C}(3 \mathrm{~F})-\mathrm{C}(2 \mathrm{~F})-\mathrm{C}(1 \mathrm{~F})$ & $105.8(8)$ \\
\hline $\mathrm{N}(1 \mathrm{~A})-\mathrm{Si}(2 \mathrm{~A})-\mathrm{N}(2 \mathrm{~A})$ & $71.38(11)$ & $\mathrm{C}(2 \mathrm{~F})-\mathrm{C}(3 \mathrm{~F})-\mathrm{C}(4 \mathrm{~F})$ & $103.8(8)$ \\
\hline $\mathrm{N}(1 \mathrm{~A})-\mathrm{Si}(2 \mathrm{~A})-\mathrm{Cl}(1 \mathrm{~A})$ & $103.28(8)$ & $\mathrm{O}(1 \mathrm{~F})-\mathrm{C}(4 \mathrm{~F})-\mathrm{C}(3 \mathrm{~F})$ & $107.0(7)$ \\
\hline
\end{tabular}



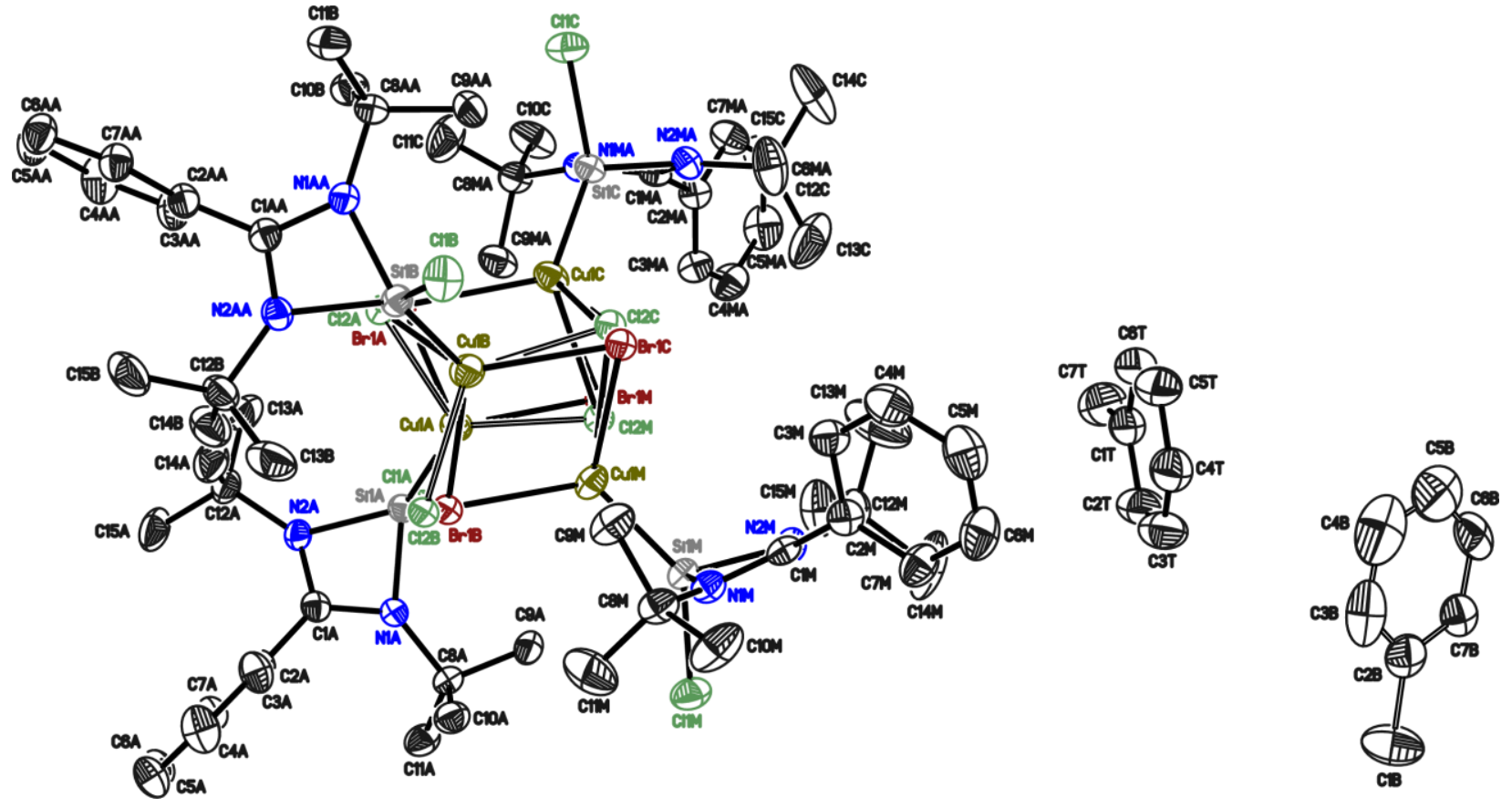

Two bromide atoms ( $\mathrm{Br} 1 \mathrm{~A} / \mathrm{Cl} 1 \mathrm{~A} ; \mathrm{Br} 1 \mathrm{M} / \mathrm{Cl} 1 \mathrm{M})$ are disordered with chloride atoms. The anisotropic displacement parameters are constrained to be identical. The occupancies of the minor positions refine to $0.047(2)$ and $0.081(2)$, respectively.

Partially bromide-chloride-disorder is supposed to stem from a halide-interchange with the chloro benzamidinato silylene NHSiCl 1.

Bond lengths $[\AA]]$ and angles [ $\left.{ }^{\circ}\right]$ for $2 b$ :

\begin{tabular}{|l|l|l|l|}
\hline $\mathrm{C}(1 \mathrm{~A})-\mathrm{N}(1 \mathrm{~A})$ & $1.342(3)$ & $\mathrm{N}(1 \mathrm{~A})-\mathrm{Si}(1 \mathrm{~A})-\mathrm{C}(1 \mathrm{~A})$ & $35.93(8)$ \\
\hline $\mathrm{C}(1 \mathrm{~A})-\mathrm{N}(2 \mathrm{~A})$ & $1.343(3)$ & $\mathrm{N}(2 \mathrm{~A})-\mathrm{Si}(1 \mathrm{~A})-\mathrm{C}(1 \mathrm{~A})$ & $35.96(8)$ \\
\hline $\mathrm{C}(1 \mathrm{~A})-\mathrm{C}(2 \mathrm{~A})$ & $1.486(3)$ & $\mathrm{Cl}(1 \mathrm{~A})-\mathrm{Si}(1 \mathrm{~A})-\mathrm{C}(1 \mathrm{~A})$ & $111.61(6)$ \\
\hline $\mathrm{C}(1 \mathrm{~A})-\mathrm{Si}(1 \mathrm{~A})$ & $2.286(2)$ & $\mathrm{Cu}(1 \mathrm{~A})-\mathrm{Si}(1 \mathrm{~A})-\mathrm{C}(1 \mathrm{~A})$ & $123.51(6)$ \\
\hline $\mathrm{Cl}(1 \mathrm{~A})-\mathrm{Si}(1 \mathrm{~A})$ & $2.1112(11)$ & $\mathrm{C}(3 \mathrm{~A})-\mathrm{C}(2 \mathrm{~A})-\mathrm{C}(7 \mathrm{~A})$ & $119.7(2)$ \\
\hline $\mathrm{Cu}(1 \mathrm{~A})-\mathrm{Si}(1 \mathrm{~A})$ & $2.2085(7)$ & $\mathrm{C}(3 \mathrm{~A})-\mathrm{C}(2 \mathrm{~A})-\mathrm{C}(1 \mathrm{~A})$ & $119.0(2)$ \\
\hline $\mathrm{Cu}(1 \mathrm{~A})-\mathrm{Cl}(2 \mathrm{M})$ & $2.427(14)$ & $\mathrm{C}(7 \mathrm{~A})-\mathrm{C}(2 \mathrm{~A})-\mathrm{C}(1 \mathrm{~A})$ & $121.2(2)$ \\
\hline $\mathrm{Cu}(1 \mathrm{~A})-\mathrm{Br}(1 \mathrm{~A})$ & $2.4638(6)$ & $\mathrm{C}(1 \mathrm{~A})-\mathrm{N}(2 \mathrm{~A})-\mathrm{C}(12 \mathrm{~A})$ & $131.2(2)$ \\
\hline $\mathrm{Cu}(1 \mathrm{~A})-\mathrm{Br}(1 \mathrm{M})$ & $2.5007(6)$ & $\mathrm{C}(1 \mathrm{~A})-\mathrm{N}(2 \mathrm{~A})-\mathrm{Si}(1 \mathrm{~A})$ & $90.48(13)$ \\
\hline $\mathrm{Cu}(1 \mathrm{~A})-\mathrm{Cl}(2 \mathrm{~A})$ & $2.57(2)$ & $\mathrm{C}(12 \mathrm{~A})-\mathrm{N}(2 \mathrm{~A})-\mathrm{Si}(1 \mathrm{~A})$ & $131.56(15)$ \\
\hline $\mathrm{Cu}(1 \mathrm{~A})-\mathrm{Br}(1 \mathrm{~A}) \# 1$ & $2.7860(10)$ & $\mathrm{N}(2 \mathrm{M})-\mathrm{C}(1 \mathrm{M})-\mathrm{N}(1 \mathrm{M})$ & $105.82(19)$ \\
\hline $\mathrm{Cu}(1 \mathrm{~A})-\mathrm{Cu}(1 \mathrm{M})$ & $2.9649(7)$ & $\mathrm{N}(2 \mathrm{M})-\mathrm{C}(1 \mathrm{M})-\mathrm{C}(2 \mathrm{M})$ & $127.2(2)$ \\
\hline $\mathrm{Br}(1 \mathrm{~A})-\mathrm{Cu}(1 \mathrm{M}) \# 1$ & $2.4978(5)$ & $\mathrm{N}(1 \mathrm{M})-\mathrm{C}(1 \mathrm{M})-\mathrm{C}(2 \mathrm{M})$ & $126.9(2)$ \\
\hline $\mathrm{N}(1 \mathrm{~A})-\mathrm{C}(8 \mathrm{~A})$ & $1.487(3)$ & $\mathrm{N}(2 \mathrm{M})-\mathrm{C}(1 \mathrm{M})-\mathrm{Si}(1 \mathrm{M})$ & $53.11(11)$ \\
\hline $\mathrm{N}(1 \mathrm{~A})-\mathrm{Si}(1 \mathrm{~A})$ & $1.8244(19)$ & $\mathrm{N}(1 \mathrm{M})-\mathrm{C}(1 \mathrm{M})-\mathrm{Si}(1 \mathrm{M})$ & $53.63(11)$ \\
\hline
\end{tabular}




\begin{tabular}{|c|c|c|c|}
\hline $\operatorname{Si}(1 A)-N(2 A)$ & $1.8390(19)$ & $C(2 M)-C(1 M)-S i(1 M)$ & $168.87(16)$ \\
\hline$C(2 A)-C(3 A)$ & $1.394(3)$ & $C(1 M)-N(1 M)-C(8 M)$ & $131.9(2)$ \\
\hline$C(2 A)-C(7 A)$ & $1.396(3)$ & $C(1 M)-N(1 M)-S i(1 M)$ & $90.50(13)$ \\
\hline$N(2 A)-C(12 A)$ & $1.480(3)$ & $C(8 M)-N(1 M)-S i(1 M)$ & $135.56(16)$ \\
\hline$C(1 M)-N(2 M)$ & $1.337(3)$ & $N(2 M)-S i(1 M)-N(1 M)$ & 71.18(9) \\
\hline$C(1 M)-N(1 M)$ & $1.339(3)$ & $\mathrm{N}(2 \mathrm{M})-\mathrm{Si}(1 \mathrm{M})-\mathrm{Cl}(1 \mathrm{M})$ & $103.03(7)$ \\
\hline$C(1 M)-C(2 M)$ & $1.488(3)$ & $\mathrm{N}(1 \mathrm{M})-\mathrm{Si}(1 \mathrm{M})-\mathrm{Cl}(1 \mathrm{M})$ & $103.77(7)$ \\
\hline$C(1 M)-S i(1 M)$ & $2.284(2)$ & $\mathrm{N}(2 \mathrm{M})-\mathrm{Si}(1 \mathrm{M})-\mathrm{Cu}(1 \mathrm{M})$ & $121.44(7)$ \\
\hline $\mathrm{N}(1 \mathrm{M})-\mathrm{C}(8 \mathrm{M})$ & $1.487(3)$ & $\mathrm{N}(1 \mathrm{M})-\mathrm{Si}(1 \mathrm{M})-\mathrm{Cu}(1 \mathrm{M})$ & $118.98(7)$ \\
\hline$N(1 M)-S i(1 M)$ & $1.839(2)$ & $\mathrm{Cl}(1 \mathrm{M})-\mathrm{Si}(1 \mathrm{M})-\mathrm{Cu}(1 \mathrm{M})$ & $125.20(4)$ \\
\hline $\operatorname{Si}(1 M)-N(2 M)$ & $1.827(2)$ & $\mathrm{N}(2 \mathrm{M})-\mathrm{Si}(1 \mathrm{M})-\mathrm{C}(1 \mathrm{M})$ & $35.81(8)$ \\
\hline $\mathrm{Si}(1 \mathrm{M})-\mathrm{Cl}(1 \mathrm{M})$ & $2.1128(9)$ & $\mathrm{N}(1 \mathrm{M})-\mathrm{Si}(1 \mathrm{M})-\mathrm{C}(1 \mathrm{M})$ & $35.87(8)$ \\
\hline $\mathrm{Si}(1 \mathrm{M})-\mathrm{Cu}(1 \mathrm{M})$ & $2.2068(7)$ & $\mathrm{Cl}(1 \mathrm{M})-\mathrm{Si}(1 \mathrm{M})-\mathrm{C}(1 \mathrm{M})$ & $111.09(6)$ \\
\hline $\mathrm{Cu}(1 \mathrm{M})-\mathrm{Cl}(2 \mathrm{M})$ & $2.162(13)$ & $\mathrm{Cu}(1 \mathrm{M})-\mathrm{Si}(1 \mathrm{M})-\mathrm{C}(1 \mathrm{M})$ & $123.69(6)$ \\
\hline $\mathrm{Cu}(1 \mathrm{M})-\mathrm{Br}(1 \mathrm{M})$ & $2.4632(8)$ & $\mathrm{Cl}(2 \mathrm{M})-\mathrm{Cu}(1 \mathrm{M})-\mathrm{Si}(1 \mathrm{M})$ & $124.0(4)$ \\
\hline $\mathrm{Cu}(1 \mathrm{M})-\mathrm{Br}(1 \mathrm{M}) \# 1$ & $2.7192(6)$ & $\mathrm{Si}(1 \mathrm{M})-\mathrm{Cu}(1 \mathrm{M})-\mathrm{Br}(1 \mathrm{M})$ & $126.70(2)$ \\
\hline$C(3 A)-C(4 A)$ & $1.389(4)$ & $\mathrm{Si}(1 \mathrm{M})-\mathrm{Cu}(1 \mathrm{M})-\mathrm{Br}(1 \mathrm{~A}) \# 1$ & $114.47(2)$ \\
\hline$C(2 M)-C(3 M)$ & $1.389(3)$ & $\mathrm{Br}(1 \mathrm{M})-\mathrm{Cu}(1 \mathrm{M})-\mathrm{Br}(1 \mathrm{~A}) \# 1$ & $114.222(16)$ \\
\hline$C(2 M)-C(7 M)$ & $1.390(3)$ & $\mathrm{Cl}(2 \mathrm{M})-\mathrm{Cu}(1 \mathrm{M})-\mathrm{Br}(1 \mathrm{M}) \# 1$ & $94.3(4)$ \\
\hline$N(2 M)-C(12 M)$ & $1.483(3)$ & $\mathrm{Si}(1 \mathrm{M})-\mathrm{Cu}(1 \mathrm{M})-\mathrm{Br}(1 \mathrm{M}) \# 1$ & $104.76(3)$ \\
\hline$C(4 A)-C(5 A)$ & $1.381(4)$ & $\operatorname{Br}(1 M)-C u(1 M)-\operatorname{Br}(1 M) \# 1$ & $89.11(3)$ \\
\hline$C(3 M)-C(4 M)$ & $1.386(4)$ & $\operatorname{Br}(1 \mathrm{~A}) \# 1-\mathrm{Cu}(1 \mathrm{M})-\operatorname{Br}(1 \mathrm{M}) \# 1$ & $97.436(15)$ \\
\hline$C(5 A)-C(6 A)$ & $1.381(4)$ & $\mathrm{Cl}(2 \mathrm{M})-\mathrm{Cu}(1 \mathrm{M})-\mathrm{Cu}(1 \mathrm{~A})$ & $53.8(4)$ \\
\hline$C(4 M)-C(5 M)$ & $1.380(4)$ & $\mathrm{Si}(1 \mathrm{M})-\mathrm{Cu}(1 \mathrm{M})-\mathrm{Cu}(1 \mathrm{~A})$ & $154.41(2)$ \\
\hline$C(6 A)-C(7 A)$ & $1.387(3)$ & $\mathrm{Br}(1 \mathrm{M})-\mathrm{Cu}(1 \mathrm{M})-\mathrm{Cu}(1 \mathrm{~A})$ & 53.906(19) \\
\hline$C(5 M)-C(6 M)$ & $1.380(4)$ & $\mathrm{Br}(1 \mathrm{~A}) \# 1-\mathrm{Cu}(1 \mathrm{M})-\mathrm{Cu}(1 \mathrm{~A})$ & $60.614(18)$ \\
\hline$C(6 M)-C(7 M)$ & $1.386(4)$ & $\mathrm{Br}(1 \mathrm{M}) \# 1-\mathrm{Cu}(1 \mathrm{M})-\mathrm{Cu}(1 \mathrm{~A})$ & $100.82(2)$ \\
\hline$C(8 A)-C(9 A)$ & $1.528(3)$ & $\mathrm{Cu}(1 \mathrm{M})-\mathrm{Br}(1 \mathrm{M})-\mathrm{Cu}(1 \mathrm{~A})$ & 73.351(14) \\
\hline$C(8 A)-C(10 A)$ & $1.529(3)$ & $\mathrm{Cu}(1 \mathrm{M})-\mathrm{Br}(1 \mathrm{M})-\mathrm{Cu}(1 \mathrm{M}) \# 1$ & $83.47(3)$ \\
\hline$C(8 A)-C(11 A)$ & $1.529(3)$ & $\mathrm{Cu}(1 \mathrm{~A})-\mathrm{Br}(1 \mathrm{M})-\mathrm{Cu}(1 \mathrm{M}) \# 1$ & $75.770(14)$ \\
\hline$C(8 M)-C(10 M)$ & $1.524(3)$ & $\mathrm{Cu}(1 \mathrm{M})-\mathrm{Cl}(2 \mathrm{M})-\mathrm{Cu}(1 \mathrm{~A})$ & $80.3(4)$ \\
\hline$C(8 M)-C(9 M)$ & $1.525(3)$ & $C(4 A)-C(3 A)-C(2 A)$ & $119.7(2)$ \\
\hline$C(8 M)-C(11 M)$ & $1.527(4)$ & $C(3 M)-C(2 M)-C(7 M)$ & $120.3(2)$ \\
\hline$C(12 A)-C(14 A)$ & $1.526(4)$ & $C(3 M)-C(2 M)-C(1 M)$ & $119.6(2)$ \\
\hline$C(12 A)-C(15 A)$ & $1.527(3)$ & $C(7 M)-C(2 M)-C(1 M)$ & $120.1(2)$ \\
\hline$C(12 A)-C(13 A)$ & $1.530(4)$ & $C(1 M)-N(2 M)-C(12 M)$ & $131.7(2)$ \\
\hline$C(12 M)-C(15 M)$ & $1.515(4)$ & $C(1 M)-N(2 M)-S i(1 M)$ & $91.08(14)$ \\
\hline$C(12 M)-C(14 M)$ & $1.517(4)$ & $C(12 M)-N(2 M)-S i(1 M)$ & $135.43(15)$ \\
\hline$C(12 M)-C(13 M)$ & $1.517(4)$ & $C(5 A)-C(4 A)-C(3 A)$ & $120.3(2)$ \\
\hline$C(7 T)-C(1 T)$ & $1.520(5)$ & $C(4 M)-C(3 M)-C(2 M)$ & $119.5(2)$ \\
\hline$C(1 T)-C(6 T)$ & $1.376(4)$ & $C(6 A)-C(5 A)-C(4 A)$ & $120.2(2)$ \\
\hline$C(1 T)-C(2 T)$ & $1.386(4)$ & $C(5 M)-C(4 M)-C(3 M)$ & $120.2(3)$ \\
\hline$C(2 T)-C(3 T)$ & $1.386(4)$ & $C(5 A)-C(6 A)-C(7 A)$ & $120.2(2)$ \\
\hline$C(3 T)-C(4 T)$ & $1.376(4)$ & $C(4 M)-C(5 M)-C(6 M)$ & $120.4(3)$ \\
\hline
\end{tabular}




\begin{tabular}{|c|c|c|c|}
\hline$C(4 T)-C(5 T)$ & $1.371(4)$ & $C(6 A)-C(7 A)-C(2 A)$ & $119.9(2)$ \\
\hline$C(5 T)-C(6 T)$ & $1.384(5)$ & $C(5 M)-C(6 M)-C(7 M)$ & $120.0(3)$ \\
\hline$C(1 B)-C(2 B)$ & $1.507(9)$ & $N(1 A)-C(8 A)-C(9 A)$ & $105.70(18)$ \\
\hline$C(2 B)-C(3 B)$ & $1.366(10)$ & $N(1 A)-C(8 A)-C(10 A)$ & $112.00(18)$ \\
\hline$C(2 B)-C(7 B)$ & $1.388(9)$ & $C(9 A)-C(8 A)-C(10 A)$ & $108.7(2)$ \\
\hline$C(3 B)-C(4 B)$ & $1.378(10)$ & $N(1 A)-C(8 A)-C(11 A)$ & $108.53(18)$ \\
\hline$C(4 B)-C(5 B)$ & $1.356(11)$ & $C(9 A)-C(8 A)-C(11 A)$ & $110.5(2)$ \\
\hline$C(5 B)-C(6 B)$ & $1.350(9)$ & $C(10 A)-C(8 A)-C(11 A)$ & 111.29(19) \\
\hline \multirow[t]{2}{*}{$C(6 B)-C(7 B)$} & $1.355(9)$ & $C(6 M)-C(7 M)-C(2 M)$ & $119.6(2)$ \\
\hline & & $N(1 M)-C(8 M)-C(10 M)$ & $111.4(2)$ \\
\hline$N(1 A)-C(1 A)-N(2 A)$ & $105.43(18)$ & $N(1 M)-C(8 M)-C(9 M)$ & $111.06(18)$ \\
\hline$N(1 A)-C(1 A)-C(2 A)$ & $127.0(2)$ & $C(10 M)-C(8 M)-C(9 M)$ & $110.8(2)$ \\
\hline$N(2 A)-C(1 A)-C(2 A)$ & $127.4(2)$ & $\mathrm{N}(1 \mathrm{M})-\mathrm{C}(8 \mathrm{M})-\mathrm{C}(11 \mathrm{M})$ & $104.6(2)$ \\
\hline$N(1 A)-C(1 A)-S i(1 A)$ & $52.93(11)$ & $C(10 M)-C(8 M)-C(11 M)$ & $109.9(2)$ \\
\hline$N(2 A)-C(1 A)-S i(1 A)$ & $53.56(11)$ & $C(9 M)-C(8 M)-C(11 M)$ & $108.9(2)$ \\
\hline$C(2 A)-C(1 A)-S i(1 A)$ & $167.28(15)$ & $N(2 A)-C(12 A)-C(14 A)$ & $110.8(2)$ \\
\hline $\mathrm{Si}(1 \mathrm{~A})-\mathrm{Cu}(1 \mathrm{~A})-\mathrm{Cl}(2 \mathrm{M})$ & $114.6(3)$ & $N(2 A)-C(12 A)-C(15 A)$ & $111.0(2)$ \\
\hline $\operatorname{Si}(1 A)-C u(1 A)-B r(1 A)$ & $131.76(2)$ & $C(14 A)-C(12 A)-C(15 A)$ & $110.6(2)$ \\
\hline $\mathrm{Si}(1 \mathrm{~A})-\mathrm{Cu}(1 \mathrm{~A})-\mathrm{Br}(1 \mathrm{M})$ & $117.24(2)$ & $N(2 A)-C(12 A)-C(13 A)$ & $104.7(2)$ \\
\hline $\operatorname{Br}(1 \mathrm{~A})-\mathrm{Cu}(1 \mathrm{~A})-\mathrm{Br}(1 \mathrm{M})$ & $104.412(16)$ & $C(14 A)-C(12 A)-C(13 A)$ & $110.0(2)$ \\
\hline $\mathrm{Si}(1 \mathrm{~A})-\mathrm{Cu}(1 \mathrm{~A})-\mathrm{Cl}(2 \mathrm{~A})$ & $124.8(5)$ & $C(15 A)-C(12 A)-C(13 A)$ & $109.5(2)$ \\
\hline $\mathrm{Cl}(2 \mathrm{M})-\mathrm{Cu}(1 \mathrm{~A})-\mathrm{Cl}(2 \mathrm{~A})$ & $118.0(6)$ & $N(2 M)-C(12 M)-C(15 M)$ & $106.4(2)$ \\
\hline $\mathrm{Si}(1 \mathrm{~A})-\mathrm{Cu}(1 \mathrm{~A})-\mathrm{Br}(1 \mathrm{~A}) \# 1$ & $103.10(3)$ & $N(2 M)-C(12 M)-C(14 M)$ & $109.3(2)$ \\
\hline $\mathrm{Br}(1 \mathrm{~A})-\mathrm{Cu}(1 \mathrm{~A})-\mathrm{Br}(1 \mathrm{~A}) \# 1$ & $88.59(2)$ & $C(15 M)-C(12 M)-C(14 M)$ & $108.9(2)$ \\
\hline $\operatorname{Br}(1 M)-C u(1 A)-B r(1 A) \# 1$ & $103.865(14)$ & $N(2 M)-C(12 M)-C(13 M)$ & $111.7(2)$ \\
\hline $\mathrm{Si}(1 \mathrm{~A})-\mathrm{Cu}(1 \mathrm{~A})-\mathrm{Cu}(1 \mathrm{M})$ & 119.41(3) & $C(15 M)-C(12 M)-C(13 M)$ & $110.0(3)$ \\
\hline $\mathrm{Cl}(2 \mathrm{M})-\mathrm{Cu}(1 \mathrm{~A})-\mathrm{Cu}(1 \mathrm{M})$ & $45.9(3)$ & $C(14 M)-C(12 M)-C(13 M)$ & $110.5(3)$ \\
\hline $\mathrm{Br}(1 \mathrm{~A})-\mathrm{Cu}(1 \mathrm{~A})-\mathrm{Cu}(1 \mathrm{M})$ & $104.53(2)$ & $C(6 T)-C(1 T)-C(2 T)$ & $117.6(3)$ \\
\hline $\mathrm{Br}(1 \mathrm{M})-\mathrm{Cu}(1 \mathrm{~A})-\mathrm{Cu}(1 \mathrm{M})$ & $52.744(14)$ & $C(6 T)-C(1 T)-C(7 T)$ & $121.4(3)$ \\
\hline $\mathrm{Cl}(2 \mathrm{~A})-\mathrm{Cu}(1 \mathrm{~A})-\mathrm{Cu}(1 \mathrm{M})$ & $108.6(5)$ & $C(2 T)-C(1 T)-C(7 T)$ & $121.0(3)$ \\
\hline $\mathrm{Br}(1 \mathrm{~A}) \# 1-\mathrm{Cu}(1 \mathrm{~A})-\mathrm{Cu}(1 \mathrm{M})$ & $51.367(16)$ & $C(3 T)-C(2 T)-C(1 T)$ & $121.6(3)$ \\
\hline $\mathrm{Cu}(1 \mathrm{~A})-\mathrm{Br}(1 \mathrm{~A})-\mathrm{Cu}(1 \mathrm{M}) \# 1$ & $80.623(14)$ & $\mathrm{C}(4 \mathrm{~T})-\mathrm{C}(3 \mathrm{~T})-\mathrm{C}(2 \mathrm{~T})$ & $119.8(3)$ \\
\hline $\mathrm{Cu}(1 \mathrm{~A})-\mathrm{Br}(1 \mathrm{~A})-\mathrm{Cu}(1 \mathrm{~A}) \# 1$ & $85.15(2)$ & $C(5 T)-C(4 T)-C(3 T)$ & $119.1(3)$ \\
\hline $\mathrm{Cu}(1 \mathrm{M}) \# 1-\mathrm{Br}(1 \mathrm{~A})-\mathrm{Cu}(1 \mathrm{~A}) \# 1$ & $68.017(12)$ & $C(4 T)-C(5 T)-C(6 T)$ & $120.9(3)$ \\
\hline$C(1 A)-N(1 A)-C(8 A)$ & $131.89(18)$ & $C(1 T)-C(6 T)-C(5 T)$ & $121.0(3)$ \\
\hline$C(1 A)-N(1 A)-S i(1 A)$ & $91.14(14)$ & $C(3 B)-C(2 B)-C(7 B)$ & $118.8(7)$ \\
\hline$C(8 A)-N(1 A)-S i(1 A)$ & $136.97(15)$ & $C(3 B)-C(2 B)-C(1 B)$ & $123.2(9)$ \\
\hline$N(1 A)-S i(1 A)-N(2 A)$ & $71.32(8)$ & $C(7 B)-C(2 B)-C(1 B)$ & $118.0(8)$ \\
\hline$N(1 A)-S i(1 A)-C l(1 A)$ & $103.18(7)$ & $C(2 B)-C(3 B)-C(4 B)$ & $121.6(9)$ \\
\hline $\mathrm{N}(2 \mathrm{~A})-\mathrm{Si}(1 \mathrm{~A})-\mathrm{Cl}(1 \mathrm{~A})$ & $103.88(6)$ & $C(5 B)-C(4 B)-C(3 B)$ & $118.3(9)$ \\
\hline$N(1 A)-S i(1 A)-C u(1 A)$ & 119.51(7) & $C(6 B)-C(5 B)-C(4 B)$ & $120.6(9)$ \\
\hline$N(2 A)-S i(1 A)-C u(1 A)$ & $121.10(6)$ & $C(5 B)-C(6 B)-C(7 B)$ & $121.8(9)$ \\
\hline $\mathrm{Cl}(1 \mathrm{~A})-\mathrm{Si}(1 \mathrm{~A})-\mathrm{Cu}(1 \mathrm{~A})$ & $124.84(4)$ & $C(6 B)-C(7 B)-C(2 B)$ & $118.8(8)$ \\
\hline
\end{tabular}




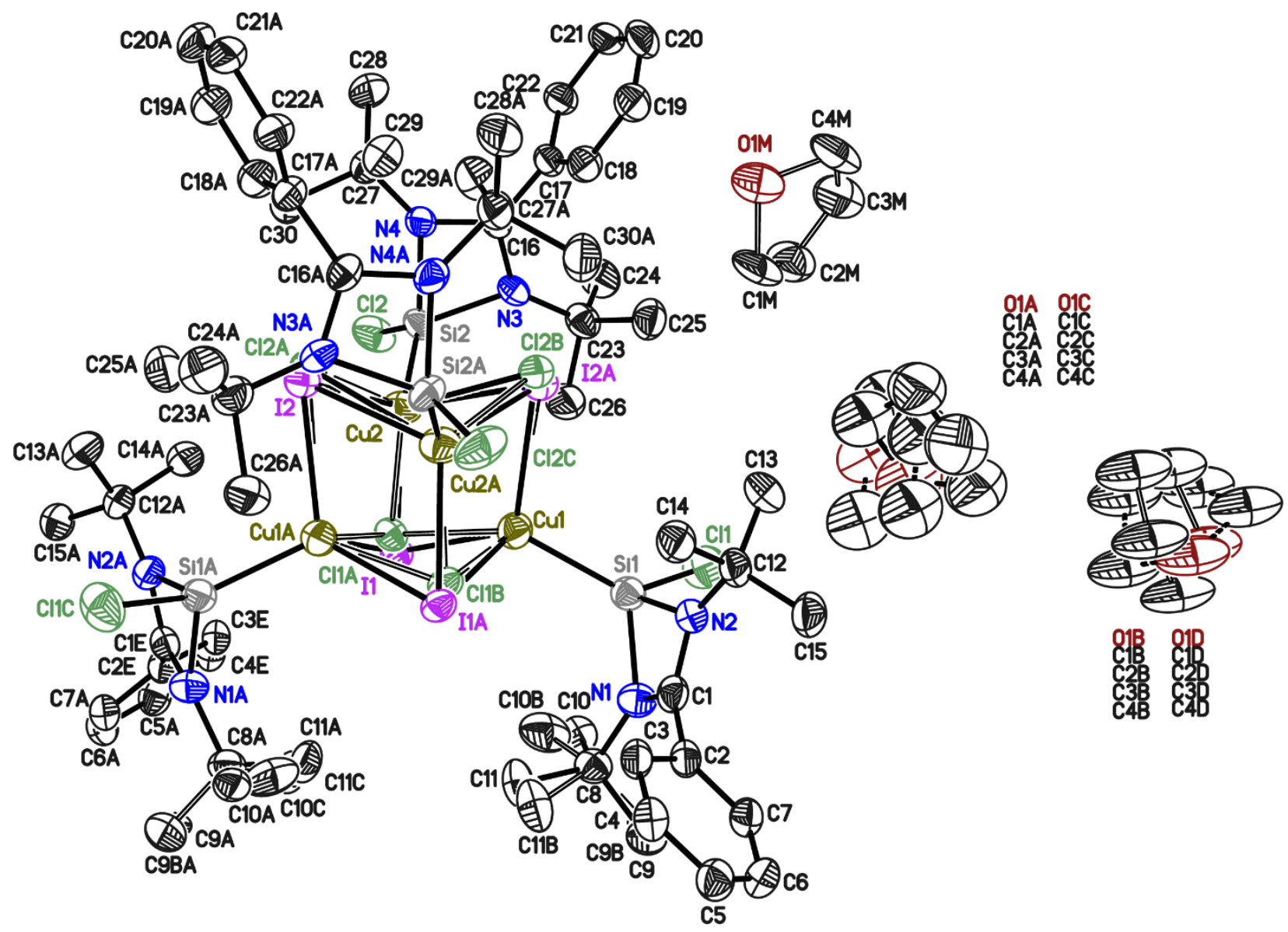

Two iodide atoms $(11 / \mathrm{Cl} 1 \mathrm{~A} ; 12 / \mathrm{Cl} 2 \mathrm{~A})$ are disordered with chloride atoms. The anisotropic displacement parameters are constrained to be identical. The occupancies of the minor positions refine to $0.125(2)$ and $0.162(2)$, respectively.

Partially iodide-chloride-disorder is supposed to stem from a halide-interchange with the chloro benzamidinato silylene $\mathrm{NHSiCl} 1$.

The carbon atoms of one tert-butyl group (C9-C11/C9B-C11B) are disordered about two positions. They are refined with distance restraints and restraints for the anisotropic displacement parameters. The occupancy of the minor position refines to $0.46(4)$.

All THF molecules are disordered. O1M-C4M is disordered about an inversion center, O1BC4B/O1D-C4D about a two-fold axis and a second position, and O1A-C4A/O1C-C4C about two 
positions. They are refined with distance restraints and restraints for the anisotropic displacement parameters. The occupancies of the minor positions refine to $0.174(7)$ and $0.362(8)$, Bond lengths $[\AA \AA]$ and angles $\left[^{\circ}\right]$ for $2 c$ :

\begin{tabular}{|c|c|c|c|}
\hline $\mathrm{I}(1)-\mathrm{Cu}(1)$ & $2.5993(9)$ & $\mathrm{Cl}(1)-\mathrm{Si}(1)-\mathrm{C}(1)$ & $113.24(12)$ \\
\hline $\mathrm{I}(1)-\mathrm{Cu}(2)$ & $2.6202(7)$ & $\mathrm{Cu}(1)-\mathrm{Si}(1)-\mathrm{C}(1)$ & $122.52(11)$ \\
\hline $\mathrm{I}(1)-\mathrm{Cu}(1) \# 1$ & $2.8559(8)$ & $\mathrm{Cu}(1) \# 1-\mathrm{I}(2)-\mathrm{Cu}(2)$ & 73.99(3) \\
\hline $\mathrm{Cl}(1 \mathrm{~A})-\mathrm{Cu}(2)$ & $2.273(15)$ & $\mathrm{Cu}(1) \# 1-\mathrm{I}(2)-\mathrm{Cu}(2) \# 1$ & $65.48(3)$ \\
\hline $\mathrm{Cl}(1 \mathrm{~A})-\mathrm{Cu}(1)$ & $2.526(17)$ & $\mathrm{Cu}(2)-\mathrm{I}(2)-\mathrm{Cu}(2) \# 1$ & $78.57(4)$ \\
\hline $\mathrm{Cl}(1 \mathrm{~A})-\mathrm{Cu}(1) \# 1$ & $2.616(17)$ & $C(7)-C(2)-C(3)$ & $119.7(4)$ \\
\hline$C(1)-N(2)$ & $1.333(5)$ & $C(7)-C(2)-C(1)$ & $121.3(4)$ \\
\hline$C(1)-N(1)$ & $1.346(5)$ & $C(3)-C(2)-C(1)$ & $118.9(4)$ \\
\hline$C(1)-C(2)$ & $1.478(6)$ & $C(1)-N(2)-C(12)$ & $132.2(4)$ \\
\hline$C(1)-\operatorname{Si}(1)$ & $2.282(4)$ & $C(1)-N(2)-S i(1)$ & $90.8(3)$ \\
\hline $\mathrm{Cl}(1)-\mathrm{Si}(1)$ & $2.0980(17)$ & $C(12)-N(2)-S i(1)$ & $135.8(3)$ \\
\hline $\mathrm{Cu}(1)-\mathrm{Si}(1)$ & $2.2285(13)$ & $\mathrm{Si}(2)-\mathrm{Cu}(2)-\mathrm{Cl}(1 \mathrm{~A})$ & $119.1(4)$ \\
\hline $\mathrm{Cu}(1)-\mathrm{I}(2) \# 1$ & $2.6221(10)$ & $\mathrm{Si}(2)-\mathrm{Cu}(2)-\mathrm{Cl}(2 \mathrm{~A})$ & $117.5(5)$ \\
\hline $\mathrm{Cu}(1)-\mathrm{Cu}(2)$ & $2.9641(9)$ & $\mathrm{Cl}(1 \mathrm{~A})-\mathrm{Cu}(2)-\mathrm{Cl}(2 \mathrm{~A})$ & $111.5(6)$ \\
\hline $\mathrm{Si}(1)-\mathrm{N}(1)$ & $1.826(4)$ & $\mathrm{Si}(2)-\mathrm{Cu}(2)-\mathrm{I}(1)$ & $116.19(4)$ \\
\hline $\mathrm{Si}(1)-\mathrm{N}(2)$ & $1.833(4)$ & $\mathrm{Si}(2)-\mathrm{Cu}(2)-\mathrm{I}(2)$ & $122.36(4)$ \\
\hline $\mathrm{I}(2)-\mathrm{Cu}(2)$ & $2.6232(13)$ & $\mathrm{I}(1)-\mathrm{Cu}(2)-\mathrm{I}(2)$ & $109.69(3)$ \\
\hline $\mathrm{I}(2)-\mathrm{Cu}(2) \# 1$ & $2.8472(14)$ & $\mathrm{Si}(2)-\mathrm{Cu}(2)-\mathrm{I}(2) \# 1$ & $102.96(4)$ \\
\hline $\mathrm{Cl}(2 \mathrm{~A})-\mathrm{Cu}(2)$ & $2.57(3)$ & $\mathrm{I}(1)-\mathrm{Cu}(2)-\mathrm{I}(2) \# 1$ & $108.59(3)$ \\
\hline$C(2)-C(7)$ & $1.384(6)$ & $\mathrm{I}(2)-\mathrm{Cu}(2)-\mathrm{I}(2) \# 1$ & $93.21(4)$ \\
\hline$C(2)-C(3)$ & $1.392(6)$ & $\mathrm{Si}(2)-\mathrm{Cu}(2)-\mathrm{Cu}(1)$ & $127.08(4)$ \\
\hline$N(2)-C(12)$ & $1.489(5)$ & $\mathrm{Cl}(1 \mathrm{~A})-\mathrm{Cu}(2)-\mathrm{Cu}(1)$ & $55.8(4)$ \\
\hline $\mathrm{Cl}(2)-\mathrm{Si}(2)$ & $2.1050(16)$ & $\mathrm{Cl}(2 \mathrm{~A})-\mathrm{Cu}(2)-\mathrm{Cu}(1)$ & $110.5(5)$ \\
\hline $\mathrm{Cu}(2)-\mathrm{Si}(2)$ & $2.2306(13)$ & $\mathrm{I}(1)-\mathrm{Cu}(2)-\mathrm{Cu}(1)$ & $55.07(2)$ \\
\hline $\mathrm{Si}(2)-N(3)$ & $1.815(3)$ & $\mathrm{I}(2)-\mathrm{Cu}(2)-\mathrm{Cu}(1)$ & 107.11(3) \\
\hline $\operatorname{Si}(2)-N(4)$ & $1.830(3)$ & $\mathrm{I}(2) \# 1-\mathrm{Cu}(2)-\mathrm{Cu}(1)$ & $53.60(2)$ \\
\hline $\mathrm{Si}(2)-C(16)$ & $2.274(4)$ & $N(3)-S i(2)-N(4)$ & $71.29(15)$ \\
\hline$N(3)-C(16)$ & $1.335(5)$ & $\mathrm{N}(3)-\mathrm{Si}(2)-\mathrm{Cl}(2)$ & $104.42(12)$ \\
\hline$N(3)-C(23)$ & $1.481(6)$ & $\mathrm{N}(4)-\mathrm{Si}(2)-\mathrm{Cl}(2)$ & $103.84(12)$ \\
\hline$C(3)-C(4)$ & $1.380(6)$ & $N(3)-S i(2)-C u(2)$ & $116.82(12)$ \\
\hline
\end{tabular}




\begin{tabular}{|c|c|c|c|}
\hline$N(4)-C(16)$ & $1.335(5)$ & $\mathrm{N}(4)-\mathrm{Si}(2)-\mathrm{Cu}(2)$ & $120.65(12)$ \\
\hline$N(4)-C(27)$ & $1.484(6)$ & $\mathrm{Cl}(2)-\mathrm{Si}(2)-\mathrm{Cu}(2)$ & $126.19(6)$ \\
\hline$C(4)-C(5)$ & $1.381(6)$ & $N(3)-S i(2)-C(16)$ & $35.94(15)$ \\
\hline$C(6)-C(7)$ & $1.380(6)$ & $N(4)-S i(2)-C(16)$ & $35.96(14)$ \\
\hline$C(6)-C(5)$ & $1.387(6)$ & $\mathrm{Cl}(2)-\mathrm{Si}(2)-\mathrm{C}(16)$ & $112.52(11)$ \\
\hline$N(1)-C(8)$ & $1.488(5)$ & $\mathrm{Cu}(2)-\mathrm{Si}(2)-\mathrm{C}(16)$ & $121.22(11)$ \\
\hline$C(8)-C(11)$ & $1.502(11)$ & $C(16)-N(3)-C(23)$ & $132.0(3)$ \\
\hline$C(8)-C(9 B)$ & $1.513(10)$ & $C(16)-N(3)-S i(2)$ & $91.1(2)$ \\
\hline$C(8)-C(9)$ & $1.525(10)$ & $C(23)-N(3)-S i(2)$ & $136.9(3)$ \\
\hline$C(8)-C(10)$ & $1.526(10)$ & $C(4)-C(3)-C(2)$ & $120.0(4)$ \\
\hline$C(8)-C(11 B)$ & $1.530(10)$ & $C(16)-N(4)-C(27)$ & $131.5(3)$ \\
\hline$C(8)-C(10 B)$ & $1.536(10)$ & $\mathrm{C}(16)-\mathrm{N}(4)-\mathrm{Si}(2)$ & $90.4(3)$ \\
\hline$C(12)-C(15)$ & $1.518(6)$ & $C(27)-N(4)-S i(2)$ & $133.2(3)$ \\
\hline$C(12)-C(13)$ & $1.525(6)$ & $C(3)-C(4)-C(5)$ & $120.2(4)$ \\
\hline$C(12)-C(14)$ & $1.526(6)$ & $C(7)-C(6)-C(5)$ & $120.2(4)$ \\
\hline$C(17)-C(18)$ & $1.387(6)$ & $C(4)-C(5)-C(6)$ & $119.9(4)$ \\
\hline$C(17)-C(22)$ & $1.387(6)$ & $C(6)-C(7)-C(2)$ & $120.1(4)$ \\
\hline$C(17)-C(16)$ & $1.490(5)$ & $C(1)-N(1)-C(8)$ & $130.9(4)$ \\
\hline$C(19)-C(20)$ & $1.377(7)$ & $C(1)-N(1)-S i(1)$ & $90.7(3)$ \\
\hline$C(19)-C(18)$ & $1.383(6)$ & $C(8)-N(1)-S i(1)$ & $133.3(3)$ \\
\hline$C(20)-C(21)$ & $1.387(7)$ & $N(1)-C(8)-C(11)$ & $110.2(10)$ \\
\hline$C(21)-C(22)$ & $1.385(6)$ & $N(1)-C(8)-C(9 B)$ & $111.5(9)$ \\
\hline$C(23)-C(24)$ & $1.516(6)$ & $N(1)-C(8)-C(9)$ & $109.0(8)$ \\
\hline$C(23)-C(25)$ & $1.522(6)$ & $C(11)-C(8)-C(9)$ & $112.6(10)$ \\
\hline$C(23)-C(26)$ & $1.537(6)$ & $N(1)-C(8)-C(10)$ & $102.3(7)$ \\
\hline$C(27)-C(29)$ & $1.506(7)$ & $C(11)-C(8)-C(10)$ & $113.2(8)$ \\
\hline$C(27)-C(28)$ & $1.528(6)$ & $C(9)-C(8)-C(10)$ & $109.0(8)$ \\
\hline$C(27)-C(30)$ & $1.539(6)$ & $N(1)-C(8)-C(11 B)$ & $111.6(9)$ \\
\hline $\mathrm{O}(1 \mathrm{M})-\mathrm{C}(1 \mathrm{M})$ & $1.474(11)$ & $C(9 B)-C(8)-C(11 B)$ & $110.3(9)$ \\
\hline$O(1 M)-C(4 M)$ & $1.498(13)$ & $N(1)-C(8)-C(10 B)$ & $108.5(6)$ \\
\hline$C(1 M)-C(2 M)$ & $1.456(13)$ & $C(9 B)-C(8)-C(10 B)$ & $108.8(8)$ \\
\hline$C(2 M)-C(3 M)$ & $1.357(16)$ & $C(11 B)-C(8)-C(10 B)$ & $105.9(8)$ \\
\hline$C(3 M)-C(4 M)$ & $1.461(13)$ & $N(2)-C(12)-C(15)$ & $111.5(3)$ \\
\hline$O(1 B)-C(4 B)$ & $1.506(14)$ & $N(2)-C(12)-C(13)$ & $105.2(4)$ \\
\hline$O(1 B)-C(1 B)$ & $1.514(15)$ & $C(15)-C(12)-C(13)$ & $109.6(4)$ \\
\hline
\end{tabular}




\begin{tabular}{|c|c|c|c|}
\hline$C(1 B)-C(2 B)$ & $1.393(14)$ & $N(2)-C(12)-C(14)$ & $110.6(3)$ \\
\hline$C(2 B)-C(3 B)$ & $1.315(18)$ & $C(15)-C(12)-C(14)$ & $110.4(4)$ \\
\hline$C(3 B)-C(4 B)$ & $1.413(15)$ & $C(13)-C(12)-C(14)$ & 109.5(4) \\
\hline $\mathrm{O}(1 \mathrm{D})-\mathrm{C}(1 \mathrm{D})$ & $1.517(16)$ & $C(18)-C(17)-C(22)$ & $120.2(4)$ \\
\hline$O(1 D)-C(4 D)$ & $1.527(16)$ & $C(18)-C(17)-C(16)$ & $119.1(4)$ \\
\hline$C(1 D)-C(2 D)$ & $1.419(16)$ & $C(22)-C(17)-C(16)$ & $120.6(4)$ \\
\hline$C(2 D)-C(3 D)$ & $1.31(2)$ & $N(3)-C(16)-N(4)$ & $105.4(3)$ \\
\hline$C(3 D)-C(4 D)$ & $1.416(16)$ & $N(3)-C(16)-C(17)$ & $128.2(4)$ \\
\hline$O(1 \mathrm{~A})-C(1 \mathrm{~A})$ & $1.517(14)$ & $N(4)-C(16)-C(17)$ & $125.9(4)$ \\
\hline$O(1 A)-C(4 A)$ & $1.518(13)$ & $N(3)-C(16)-S i(2)$ & $52.95(19)$ \\
\hline$C(1 A)-C(2 A)$ & $1.367(14)$ & $N(4)-C(16)-S i(2)$ & $53.6(2)$ \\
\hline$C(2 A)-C(3 A)$ & $1.266(16)$ & $C(17)-C(16)-S i(2)$ & $165.3(3)$ \\
\hline$C(3 A)-C(4 A)$ & $1.417(13)$ & $C(20)-C(19)-C(18)$ & $120.5(4)$ \\
\hline $\mathrm{O}(1 \mathrm{C})-\mathrm{C}(4 \mathrm{C})$ & $1.533(15)$ & $C(19)-C(18)-C(17)$ & $119.6(4)$ \\
\hline $\mathrm{O}(1 \mathrm{C})-\mathrm{C}(1 \mathrm{C})$ & $1.538(16)$ & $C(19)-C(20)-C(21)$ & $120.0(4)$ \\
\hline$C(1 C)-C(2 C)$ & $1.437(16)$ & $C(22)-C(21)-C(20)$ & $120.0(4)$ \\
\hline$C(2 C)-C(3 C)$ & $1.322(19)$ & $C(21)-C(22)-C(17)$ & $119.7(4)$ \\
\hline \multirow[t]{2}{*}{$C(3 C)-C(4 C)$} & $1.412(15)$ & $N(3)-C(23)-C(24)$ & $109.1(4)$ \\
\hline & & $N(3)-C(23)-C(25)$ & $112.0(3)$ \\
\hline $\mathrm{Cu}(1)-\mathrm{I}(1)-\mathrm{Cu}(2)$ & $69.20(2)$ & $C(24)-C(23)-C(25)$ & $110.8(4)$ \\
\hline $\mathrm{Cu}(1)-\mathrm{I}(1)-\mathrm{Cu}(1) \# 1$ & $77.50(3)$ & $N(3)-C(23)-C(26)$ & $105.6(3)$ \\
\hline $\mathrm{Cu}(2)-\mathrm{I}(1)-\mathrm{Cu}(1) \# 1$ & $70.240(18)$ & $C(24)-C(23)-C(26)$ & $110.6(4)$ \\
\hline $\mathrm{Cu}(2)-\mathrm{Cl}(1 \mathrm{~A})-\mathrm{Cu}(1)$ & $76.1(5)$ & $C(25)-C(23)-C(26)$ & $108.5(4)$ \\
\hline $\mathrm{Cu}(2)-\mathrm{Cl}(1 \mathrm{~A})-\mathrm{Cu}(1) \# 1$ & $80.1(4)$ & $N(4)-C(27)-C(29)$ & $110.8(3)$ \\
\hline $\mathrm{Cu}(1)-\mathrm{Cl}(1 \mathrm{~A})-\mathrm{Cu}(1) \# 1$ & $83.4(5)$ & $N(4)-C(27)-C(28)$ & $110.2(4)$ \\
\hline$N(2)-C(1)-N(1)$ & $105.2(4)$ & $C(29)-C(27)-C(28)$ & $112.3(4)$ \\
\hline$N(2)-C(1)-C(2)$ & $126.3(4)$ & $N(4)-C(27)-C(30)$ & $104.8(4)$ \\
\hline$N(1)-C(1)-C(2)$ & $128.4(4)$ & $C(29)-C(27)-C(30)$ & $109.5(4)$ \\
\hline$N(2)-C(1)-S i(1)$ & $53.4(2)$ & $C(28)-C(27)-C(30)$ & $109.0(4)$ \\
\hline$N(1)-C(1)-S i(1)$ & $53.1(2)$ & $C(1 M)-O(1 M)-C(4 M)$ & $99.4(8)$ \\
\hline$C(2)-C(1)-S i(1)$ & $166.8(3)$ & $C(2 M)-C(1 M)-O(1 M)$ & 105.1(9) \\
\hline $\mathrm{Si}(1)-\mathrm{Cu}(1)-\mathrm{Cl}(1 \mathrm{~A})$ & $131.8(3)$ & $C(3 M)-C(2 M)-C(1 M)$ & $108.4(10)$ \\
\hline $\mathrm{Si}(1)-\mathrm{Cu}(1)-\mathrm{I}(1)$ & $123.56(4)$ & $C(2 M)-C(3 M)-C(4 M)$ & $107.9(10)$ \\
\hline $\mathrm{Si}(1)-\mathrm{Cu}(1)-\mathrm{Cl}(1 \mathrm{~A}) \# 1$ & $108.3(4)$ & $C(3 M)-C(4 M)-O(1 M)$ & $105.3(9)$ \\
\hline $\mathrm{I}(1)-\mathrm{Cu}(1)-\mathrm{Cl}(1 \mathrm{~A}) \# 1$ & $96.1(4)$ & $C(4 B)-O(1 B)-C(1 B)$ & $101.7(12)$ \\
\hline
\end{tabular}




\begin{tabular}{|l|r|l|r|}
\hline $\mathrm{Si}(1)-\mathrm{Cu}(1)-\mathrm{I}(2) \# 1$ & $110.20(4)$ & $\mathrm{C}(2 \mathrm{~B})-\mathrm{C}(1 \mathrm{~B})-\mathrm{O}(1 \mathrm{~B})$ & $105.7(13)$ \\
\hline $\mathrm{I}(1)-\mathrm{Cu}(1)-\mathrm{I}(2) \# 1$ & $116.56(3)$ & $\mathrm{C}(3 \mathrm{~B})-\mathrm{C}(2 \mathrm{~B})-\mathrm{C}(1 \mathrm{~B})$ & $112.3(14)$ \\
\hline $\mathrm{Si}(1)-\mathrm{Cu}(1)-\mathrm{I}(1) \# 1$ & $105.18(4)$ & $\mathrm{C}(2 \mathrm{~B})-\mathrm{C}(3 \mathrm{~B})-\mathrm{C}(4 \mathrm{~B})$ & $109.4(13)$ \\
\hline $\mathrm{I}(1)-\mathrm{Cu}(1)-\mathrm{I}(1) \# 1$ & $93.67(3)$ & $\mathrm{C}(3 \mathrm{~B})-\mathrm{C}(4 \mathrm{~B})-\mathrm{O}(1 \mathrm{~B})$ & $107.6(12)$ \\
\hline $\mathrm{I}(2) \# 1-\mathrm{Cu}(1)-\mathrm{I}(1) \# 1$ & $102.91(3)$ & $\mathrm{C}(1 \mathrm{D})-\mathrm{O}(1 \mathrm{D})-\mathrm{C}(4 \mathrm{D})$ & $97.7(16)$ \\
\hline $\mathrm{Si}(1)-\mathrm{Cu}(1)-\mathrm{Cu}(2)$ & $152.00(4)$ & $\mathrm{C}(2 \mathrm{D})-\mathrm{C}(1 \mathrm{D})-\mathrm{O}(1 \mathrm{D})$ & $105.8(14)$ \\
\hline $\mathrm{Cl}(1 \mathrm{~A})-\mathrm{Cu}(1)-\mathrm{Cu}(2)$ & $48.1(3)$ & $\mathrm{C}(3 \mathrm{D})-\mathrm{C}(2 \mathrm{D})-\mathrm{C}(1 \mathrm{D})$ & $107.2(17)$ \\
\hline $\mathrm{I}(1)-\mathrm{Cu}(1)-\mathrm{Cu}(2)$ & $55.73(2)$ & $\mathrm{C}(2 \mathrm{D})-\mathrm{C}(3 \mathrm{D})-\mathrm{C}(4 \mathrm{D})$ & $102.8(19)$ \\
\hline $\mathrm{Cl}(1 \mathrm{~A}) \# 1-\mathrm{Cu}(1)-\mathrm{Cu}(2)$ & $99.4(4)$ & $\mathrm{C}(3 \mathrm{D})-\mathrm{C}(4 \mathrm{D})-\mathrm{O}(1 \mathrm{D})$ & $101.1(16)$ \\
\hline $\mathrm{I}(2) \# 1-\mathrm{Cu}(1)-\mathrm{Cu}(2)$ & $60.92(3)$ & $\mathrm{C}(1 \mathrm{~A})-\mathrm{O}(1 \mathrm{~A})-\mathrm{C}(4 \mathrm{~A})$ & $100.8(10)$ \\
\hline $\mathrm{I}(1) \# 1-\mathrm{Cu}(1)-\mathrm{Cu}(2)$ & $102.75(2)$ & $\mathrm{C}(2 \mathrm{~A})-\mathrm{C}(1 \mathrm{~A})-\mathrm{O}(1 \mathrm{~A})$ & $105.1(11)$ \\
\hline $\mathrm{N}(1)-\mathrm{Si}(1)-\mathrm{N}(2)$ & $71.14(16)$ & $\mathrm{C}(3 \mathrm{~A})-\mathrm{C}(2 \mathrm{~A})-\mathrm{C}(1 \mathrm{~A})$ & $105.9(13)$ \\
\hline $\mathrm{N}(1)-\mathrm{Si}(1)-\mathrm{Cl}(1)$ & $103.51(13)$ & $\mathrm{C}(2 \mathrm{~A})-\mathrm{C}(3 \mathrm{~A})-\mathrm{C}(4 \mathrm{~A})$ & $108.4(13)$ \\
\hline $\mathrm{N}(2)-\mathrm{Si}(1)-\mathrm{Cl}(1)$ & $105.15(13)$ & $\mathrm{C}(3 \mathrm{~A})-\mathrm{C}(4 \mathrm{~A})-\mathrm{O}(1 \mathrm{~A})$ & $100.9(10)$ \\
\hline $\mathrm{N}(1)-\mathrm{Si}(1)-\mathrm{Cu}(1)$ & $122.08(13)$ & $\mathrm{C}(4 \mathrm{C})-\mathrm{O}(1 \mathrm{C})-\mathrm{C}(1 \mathrm{C})$ & $92.2(14)$ \\
\hline $\mathrm{N}(2)-\mathrm{Si}(1)-\mathrm{Cu}(1)$ & $118.12(12)$ & $\mathrm{C}(2 \mathrm{C})-\mathrm{C}(1 \mathrm{C})-\mathrm{O}(1 \mathrm{C})$ & $107.0(15)$ \\
\hline $\mathrm{CI}(1)-\mathrm{Si}(1)-\mathrm{Cu}(1)$ & $124.20(7)$ & $\mathrm{C}(3 \mathrm{C})-\mathrm{C}(2 \mathrm{C})-\mathrm{C}(1 \mathrm{C})$ & $108.8(14)$ \\
\hline $\mathrm{N}(1)-\mathrm{Si}(1)-\mathrm{C}(1)$ & $36.13(15)$ & $\mathrm{C}(2 \mathrm{C})-\mathrm{C}(3 \mathrm{C})-\mathrm{C}(4 \mathrm{C})$ & $107.3(15)$ \\
\hline $\mathrm{N}(2)-\mathrm{Si}(1)-\mathrm{C}(1)$ & $35.75(15)$ & $\mathrm{C}(3 \mathrm{C})-\mathrm{C}(4 \mathrm{C})-\mathrm{O}(1 \mathrm{C})$ & $110.9(15)$ \\
\hline
\end{tabular}




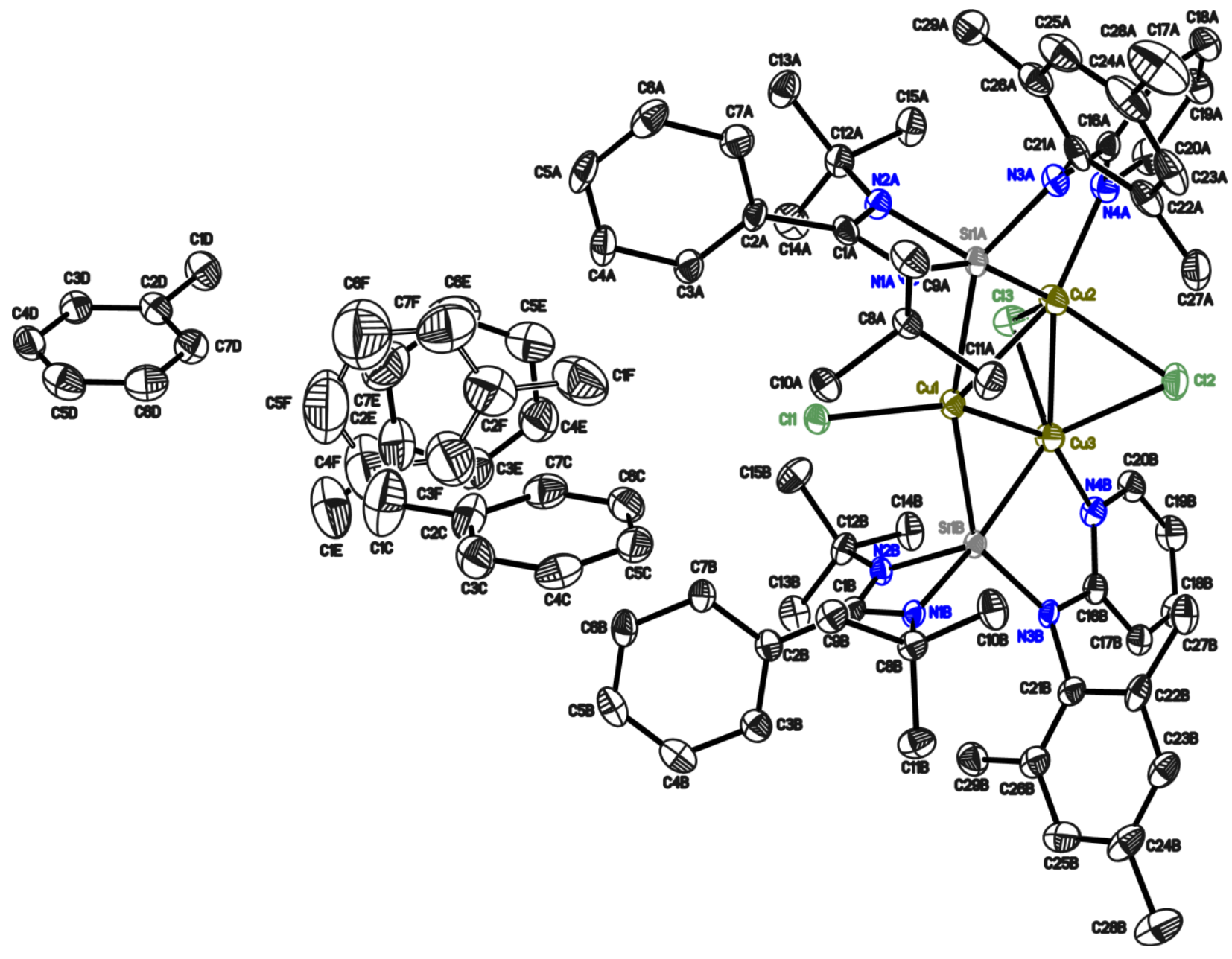

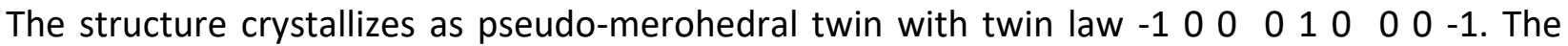
fractional contribution of the minor domain refines to $0.3242(12)$.

The carbon atoms of one toluene molecule (C1E-C7E/C1F-C7F) are disordered about two positions. They are refined with distance restraints and restraints for the anisotropic displacement parameters. The occupancy of the minor position refines to $0.398(10)$.

Bond lengths $[\AA ̊]$ and angles $\left[^{\circ}\right]$ for $3 a$ :

\begin{tabular}{|l|l|l|l|}
\hline $\mathrm{Cu}(1)-\mathrm{Cl}(1)$ & $2.2240(11)$ & $\mathrm{N}(1 \mathrm{~A})-\mathrm{Si}(1 \mathrm{~A})-\mathrm{C}(1 \mathrm{~A})$ & $35.98(17)$ \\
\hline $\mathrm{Cu}(1)-\mathrm{Cu}(3)$ & $2.4305(10)$ & $\mathrm{Cu}(2)-\mathrm{Si}(1 \mathrm{~A})-\mathrm{C}(1 \mathrm{~A})$ & $151.19(13)$ \\
\hline $\mathrm{Cu}(1)-\mathrm{Cu}(2)$ & $2.4399(10)$ & $\mathrm{N}(2 \mathrm{~A})-\mathrm{Si}(1 \mathrm{~A})-\mathrm{Cu}(1)$ & $106.11(13)$ \\
\hline $\mathrm{Cu}(1)-\mathrm{Si}(1 \mathrm{~B})$ & $2.5427(13)$ & $\mathrm{N}(3 \mathrm{~A})-\mathrm{Si}(1 \mathrm{~A})-\mathrm{Cu}(1)$ & $146.40(14)$ \\
\hline $\mathrm{Cu}(1)-\mathrm{Si}(1 \mathrm{~A})$ & $2.5569(14)$ & $\mathrm{N}(1 \mathrm{~A})-\mathrm{Si}(1 \mathrm{~A})-\mathrm{Cu}(1)$ & $101.75(13)$ \\
\hline $\mathrm{Cu}(2)-\mathrm{N}(4 \mathrm{~A})$ & $1.998(4)$ & $\mathrm{Cu}(2)-\mathrm{Si}(1 \mathrm{~A})-\mathrm{Cu}(1)$ & $60.53(4)$ \\
\hline $\mathrm{Cu}(2)-\mathrm{Si}(1 \mathrm{~A})$ & $2.2564(14)$ & $\mathrm{C}(1 \mathrm{~A})-\mathrm{Si}(1 \mathrm{~A})-\mathrm{Cu}(1)$ & $103.77(12)$ \\
\hline
\end{tabular}




\begin{tabular}{|c|c|c|c|}
\hline $\mathrm{Cu}(2)-\mathrm{Cl}(2)$ & $2.3699(13)$ & $N(2 A)-C(1 A)-N(1 A)$ & $106.8(4)$ \\
\hline $\mathrm{Cu}(2)-\mathrm{Cl}(3)$ & $2.4333(13)$ & $N(2 A)-C(1 A)-C(2 A)$ & $124.9(4)$ \\
\hline $\mathrm{Cu}(2)-\mathrm{Cu}(3)$ & $2.5078(7)$ & $N(1 A)-C(1 A)-C(2 A)$ & $128.2(4)$ \\
\hline $\mathrm{Cl}(2)-\mathrm{Cu}(3)$ & $2.4082(13)$ & $N(2 A)-C(1 A)-S i(1 A)$ & $52.9(2)$ \\
\hline $\mathrm{Cu}(3)-\mathrm{N}(4 \mathrm{~B})$ & $1.995(4)$ & $N(1 A)-C(1 A)-S i(1 A)$ & $54.4(2)$ \\
\hline $\mathrm{Cu}(3)-\mathrm{Si}(1 \mathrm{~B})$ & $2.2647(15)$ & $C(2 A)-C(1 A)-S i(1 A)$ & $174.2(3)$ \\
\hline $\mathrm{Cu}(3)-\mathrm{Cl}(3)$ & $2.3906(13)$ & $C(1 A)-N(1 A)-C(8 A)$ & $128.9(4)$ \\
\hline $\mathrm{Si}(1 \mathrm{~A})-\mathrm{N}(2 \mathrm{~A})$ & $1.820(4)$ & $C(1 A)-N(1 A)-S i(1 A)$ & $89.6(3)$ \\
\hline $\mathrm{Si}(1 \mathrm{~A})-\mathrm{N}(3 \mathrm{~A})$ & $1.828(4)$ & $C(8 A)-N(1 A)-S i(1 A)$ & $139.2(3)$ \\
\hline $\mathrm{Si}(1 \mathrm{~A})-\mathrm{N}(1 \mathrm{~A})$ & $1.854(4)$ & $\mathrm{N}(3 \mathrm{~B})-\mathrm{Si}(1 \mathrm{~B})-\mathrm{N}(2 \mathrm{~B})$ & $103.82(18)$ \\
\hline $\operatorname{Si}(1 A)-C(1 A)$ & $2.280(5)$ & $\mathrm{N}(3 \mathrm{~B})-\mathrm{Si}(1 \mathrm{~B})-\mathrm{N}(1 \mathrm{~B})$ & $101.17(18)$ \\
\hline$C(1 A)-N(2 A)$ & $1.331(6)$ & $N(2 B)-S i(1 B)-N(1 B)$ & $71.28(17)$ \\
\hline$C(1 A)-N(1 A)$ & $1.340(6)$ & $\mathrm{N}(3 \mathrm{~B})-\mathrm{Si}(1 \mathrm{~B})-\mathrm{Cu}(3)$ & $91.29(13)$ \\
\hline$C(1 A)-C(2 A)$ & $1.494(6)$ & $\mathrm{N}(2 \mathrm{~B})-\mathrm{Si}(1 \mathrm{~B})-\mathrm{Cu}(3)$ & $121.14(13)$ \\
\hline$N(1 A)-C(8 A)$ & $1.496(6)$ & $\mathrm{N}(1 \mathrm{~B})-\mathrm{Si}(1 \mathrm{~B})-\mathrm{Cu}(3)$ & 159.95(13) \\
\hline $\mathrm{Si}(1 \mathrm{~B})-\mathrm{N}(3 \mathrm{~B})$ & $1.813(4)$ & $N(3 B)-S i(1 B)-C(1 B)$ & $108.86(17)$ \\
\hline $\mathrm{Si}(1 \mathrm{~B})-\mathrm{N}(2 \mathrm{~B})$ & $1.845(4)$ & $\mathrm{N}(2 \mathrm{~B})-\mathrm{Si}(1 \mathrm{~B})-\mathrm{C}(1 \mathrm{~B})$ & $35.73(17)$ \\
\hline $\mathrm{Si}(1 \mathrm{~B})-\mathrm{N}(1 \mathrm{~B})$ & $1.856(4)$ & $\mathrm{N}(1 \mathrm{~B})-\mathrm{Si}(1 \mathrm{~B})-\mathrm{C}(1 \mathrm{~B})$ & $35.84(17)$ \\
\hline $\mathrm{Si}(1 \mathrm{~B})-\mathrm{C}(1 \mathrm{~B})$ & $2.298(5)$ & $\mathrm{Cu}(3)-\mathrm{Si}(1 \mathrm{~B})-\mathrm{C}(1 \mathrm{~B})$ & $151.50(13)$ \\
\hline$C(1 B)-N(2 B)$ & $1.342(6)$ & $\mathrm{N}(3 \mathrm{~B})-\mathrm{Si}(1 \mathrm{~B})-\mathrm{Cu}(1)$ & $145.58(14)$ \\
\hline$C(1 B)-N(1 B)$ & $1.346(6)$ & $\mathrm{N}(2 \mathrm{~B})-\mathrm{Si}(1 \mathrm{~B})-\mathrm{Cu}(1)$ & $107.56(13)$ \\
\hline$C(1 B)-C(2 B)$ & $1.488(7)$ & $\mathrm{N}(1 \mathrm{~B})-\mathrm{Si}(1 \mathrm{~B})-\mathrm{Cu}(1)$ & $101.96(12)$ \\
\hline $\mathrm{N}(1 \mathrm{~B})-\mathrm{C}(8 \mathrm{~B})$ & $1.496(6)$ & $\mathrm{Cu}(3)-\mathrm{Si}(1 \mathrm{~B})-\mathrm{Cu}(1)$ & $60.41(4)$ \\
\hline$N(2 A)-C(12 A)$ & $1.489(6)$ & $\mathrm{C}(1 \mathrm{~B})-\mathrm{Si}(1 \mathrm{~B})-\mathrm{Cu}(1)$ & $104.84(12)$ \\
\hline$C(2 A)-C(7 A)$ & $1.390(7)$ & $N(2 B)-C(1 B)-N(1 B)$ & $106.7(4)$ \\
\hline$C(2 A)-C(3 A)$ & $1.397(7)$ & $\mathrm{N}(2 \mathrm{~B})-\mathrm{C}(1 \mathrm{~B})-\mathrm{C}(2 \mathrm{~B})$ & $125.0(4)$ \\
\hline $\mathrm{N}(2 \mathrm{~B})-\mathrm{C}(12 \mathrm{~B})$ & $1.484(6)$ & $\mathrm{N}(1 \mathrm{~B})-\mathrm{C}(1 \mathrm{~B})-\mathrm{C}(2 \mathrm{~B})$ & $128.3(4)$ \\
\hline$C(2 B)-C(7 B)$ & $1.391(7)$ & $\mathrm{N}(2 \mathrm{~B})-\mathrm{C}(1 \mathrm{~B})-\mathrm{Si}(1 \mathrm{~B})$ & $53.4(2)$ \\
\hline$C(2 B)-C(3 B)$ & $1.393(7)$ & $\mathrm{N}(1 \mathrm{~B})-\mathrm{C}(1 \mathrm{~B})-\mathrm{Si}(1 \mathrm{~B})$ & $53.8(2)$ \\
\hline$N(3 A)-C(16 A)$ & $1.399(6)$ & $\mathrm{C}(2 \mathrm{~B})-\mathrm{C}(1 \mathrm{~B})-\mathrm{Si}(1 \mathrm{~B})$ & $174.3(3)$ \\
\hline$N(3 A)-C(21 A)$ & $1.447(6)$ & $C(1 B)-N(1 B)-C(8 B)$ & $128.9(4)$ \\
\hline$C(3 A)-C(4 A)$ & $1.391(7)$ & $C(1 B)-N(1 B)-S i(1 B)$ & $90.3(3)$ \\
\hline$N(4 A)-C(16 A)$ & $1.349(6)$ & $C(8 B)-N(1 B)-S i(1 B)$ & $138.4(3)$ \\
\hline$N(4 A)-C(20 A)$ & $1.351(6)$ & $C(1 A)-N(2 A)-C(12 A)$ & $131.7(4)$ \\
\hline$N(3 B)-C(16 B)$ & $1.384(6)$ & $C(1 A)-N(2 A)-S i(1 A)$ & $91.4(3)$ \\
\hline$N(3 B)-C(21 B)$ & $1.441(6)$ & $C(12 A)-N(2 A)-S i(1 A)$ & $136.0(3)$ \\
\hline$C(3 B)-C(4 B)$ & $1.392(8)$ & $C(7 A)-C(2 A)-C(3 A)$ & $120.8(4)$ \\
\hline$C(5 A)-C(4 A)$ & $1.388(8)$ & $C(7 A)-C(2 A)-C(1 A)$ & $118.4(4)$ \\
\hline$C(5 A)-C(6 A)$ & $1.389(9)$ & $C(3 A)-C(2 A)-C(1 A)$ & $120.5(4)$ \\
\hline $\mathrm{N}(4 \mathrm{~B})-\mathrm{C}(16 \mathrm{~B})$ & $1.338(6)$ & $C(1 B)-N(2 B)-C(12 B)$ & $131.5(4)$ \\
\hline$N(4 B)-C(20 B)$ & $1.351(6)$ & $C(1 B)-N(2 B)-S i(1 B)$ & $90.9(3)$ \\
\hline$C(4 B)-C(5 B)$ & $1.379(8)$ & $\mathrm{C}(12 \mathrm{~B})-\mathrm{N}(2 \mathrm{~B})-\mathrm{Si}(1 \mathrm{~B})$ & $136.8(3)$ \\
\hline$C(6 A)-C(7 A)$ & $1.384(7)$ & $C(7 B)-C(2 B)-C(3 B)$ & $119.6(5)$ \\
\hline$C(5 B)-C(6 B)$ & $1.377(8)$ & $C(7 B)-C(2 B)-C(1 B)$ & $120.3(4)$ \\
\hline
\end{tabular}




\begin{tabular}{|c|c|c|c|}
\hline$C(6 B)-C(7 B)$ & $1.384(7)$ & $C(3 B)-C(2 B)-C(1 B)$ & $119.8(4)$ \\
\hline$C(8 A)-C(11 A)$ & $1.506(7)$ & $C(16 A)-N(3 A)-C(21 A)$ & $115.1(4)$ \\
\hline$C(8 A)-C(10 A)$ & $1.533(6)$ & $C(16 A)-N(3 A)-S i(1 A)$ & $119.1(3)$ \\
\hline$C(8 A)-C(9 A)$ & $1.536(6)$ & $C(21 A)-N(3 A)-S i(1 A)$ & $125.4(3)$ \\
\hline$C(8 B)-C(9 B)$ & $1.530(6)$ & $C(4 A)-C(3 A)-C(2 A)$ & $119.5(5)$ \\
\hline$C(8 B)-C(10 B)$ & $1.530(7)$ & $C(16 A)-N(4 A)-C(20 A)$ & $119.2(4)$ \\
\hline$C(8 B)-C(11 B)$ & $1.533(6)$ & $C(16 A)-N(4 A)-C u(2)$ & $117.8(3)$ \\
\hline$C(12 A)-C(13 A)$ & $1.519(7)$ & $C(20 A)-N(4 A)-C u(2)$ & $121.8(3)$ \\
\hline$C(12 A)-C(14 A)$ & $1.528(7)$ & $C(16 B)-N(3 B)-C(21 B)$ & $115.4(4)$ \\
\hline$C(12 A)-C(15 A)$ & $1.536(7)$ & $C(16 B)-N(3 B)-S i(1 B)$ & $119.6(3)$ \\
\hline$C(12 B)-C(15 B)$ & $1.524(7)$ & $C(21 B)-N(3 B)-S i(1 B)$ & $124.4(3)$ \\
\hline$C(12 B)-C(14 B)$ & $1.524(7)$ & $C(4 B)-C(3 B)-C(2 B)$ & $120.3(5)$ \\
\hline$C(12 B)-C(13 B)$ & $1.525(7)$ & $C(4 A)-C(5 A)-C(6 A)$ & $121.5(5)$ \\
\hline$C(16 A)-C(17 A)$ & $1.400(7)$ & $C(16 B)-N(4 B)-C(20 B)$ & $119.7(4)$ \\
\hline$C(17 A)-C(18 A)$ & $1.374(7)$ & $C(16 B)-N(4 B)-C u(3)$ & $117.0(3)$ \\
\hline$C(16 B)-C(17 B)$ & $1.400(7)$ & $\mathrm{C}(20 \mathrm{~B})-\mathrm{N}(4 \mathrm{~B})-\mathrm{Cu}(3)$ & $122.4(3)$ \\
\hline$C(18 A)-C(19 A)$ & $1.395(7)$ & $C(5 B)-C(4 B)-C(3 B)$ & $119.7(5)$ \\
\hline$C(17 B)-C(18 B)$ & $1.388(7)$ & $C(7 A)-C(6 A)-C(5 A)$ & $119.4(5)$ \\
\hline$C(19 A)-C(20 A)$ & $1.381(7)$ & $C(6 B)-C(5 B)-C(4 B)$ & $119.8(5)$ \\
\hline$C(18 B)-C(19 B)$ & $1.370(8)$ & $C(6 A)-C(7 A)-C(2 A)$ & $119.7(5)$ \\
\hline$C(19 B)-C(20 B)$ & $1.363(7)$ & $C(5 B)-C(6 B)-C(7 B)$ & $121.4(5)$ \\
\hline$C(21 A)-C(26 A)$ & $1.394(7)$ & $N(1 A)-C(8 A)-C(11 A)$ & $106.3(4)$ \\
\hline$C(21 A)-C(22 A)$ & $1.402(7)$ & $N(1 A)-C(8 A)-C(10 A)$ & $112.3(4)$ \\
\hline$C(22 A)-C(23 A)$ & $1.407(7)$ & $C(11 A)-C(8 A)-C(10 A)$ & $108.4(4)$ \\
\hline$C(22 A)-C(27 A)$ & $1.493(7)$ & $N(1 A)-C(8 A)-C(9 A)$ & $109.9(4)$ \\
\hline$C(21 B)-C(26 B)$ & $1.397(7)$ & $C(11 A)-C(8 A)-C(9 A)$ & $109.3(4)$ \\
\hline$C(21 B)-C(22 B)$ & $1.411(7)$ & $C(10 A)-C(8 A)-C(9 A)$ & $110.5(4)$ \\
\hline$C(23 A)-C(24 A)$ & $1.378(9)$ & $C(6 B)-C(7 B)-C(2 B)$ & $119.1(5)$ \\
\hline$C(22 B)-C(23 B)$ & $1.385(7)$ & $N(1 B)-C(8 B)-C(9 B)$ & $112.3(4)$ \\
\hline$C(22 B)-C(27 B)$ & $1.507(7)$ & $\mathrm{N}(1 \mathrm{~B})-\mathrm{C}(8 \mathrm{~B})-\mathrm{C}(10 \mathrm{~B})$ & $107.0(4)$ \\
\hline$C(24 A)-C(25 A)$ & $1.379(9)$ & $C(9 B)-C(8 B)-C(10 B)$ & $109.1(4)$ \\
\hline$C(24 A)-C(28 A)$ & $1.516(8)$ & $\mathrm{N}(1 \mathrm{~B})-\mathrm{C}(8 \mathrm{~B})-\mathrm{C}(11 \mathrm{~B})$ & $109.4(4)$ \\
\hline$C(23 B)-C(24 B)$ & $1.385(8)$ & $C(9 B)-C(8 B)-C(11 B)$ & $109.4(4)$ \\
\hline$C(25 A)-C(26 A)$ & $1.395(7)$ & $C(10 B)-C(8 B)-C(11 B)$ & $109.6(4)$ \\
\hline$C(24 B)-C(25 B)$ & $1.397(8)$ & $N(2 A)-C(12 A)-C(13 A)$ & $111.0(4)$ \\
\hline$C(24 B)-C(28 B)$ & $1.495(7)$ & $N(2 A)-C(12 A)-C(14 A)$ & $110.8(4)$ \\
\hline$C(26 A)-C(29 A)$ & $1.494(8)$ & $C(13 A)-C(12 A)-C(14 A)$ & $110.9(4)$ \\
\hline$C(25 B)-C(26 B)$ & $1.398(7)$ & $N(2 A)-C(12 A)-C(15 A)$ & $105.9(4)$ \\
\hline$C(26 B)-C(29 B)$ & $1.504(8)$ & $C(13 A)-C(12 A)-C(15 A)$ & $109.0(4)$ \\
\hline$C(1 C)-C(2 C)$ & $1.507(7)$ & $C(14 A)-C(12 A)-C(15 A)$ & $109.2(4)$ \\
\hline$C(2 C)-C(3 C)$ & $1.383(6)$ & $N(2 B)-C(12 B)-C(15 B)$ & $109.7(4)$ \\
\hline$C(2 C)-C(7 C)$ & $1.400(6)$ & $N(2 B)-C(12 B)-C(14 B)$ & $105.9(4)$ \\
\hline$C(3 C)-C(4 C)$ & $1.383(6)$ & $C(15 B)-C(12 B)-C(14 B)$ & $108.8(4)$ \\
\hline$C(4 C)-C(5 C)$ & $1.381(6)$ & $N(2 B)-C(12 B)-C(13 B)$ & $111.9(4)$ \\
\hline
\end{tabular}




\begin{tabular}{|c|c|c|c|}
\hline$C(5 C)-C(6 C)$ & $1.376(6)$ & $C(15 B)-C(12 B)-C(13 B)$ & $110.5(4)$ \\
\hline$C(6 C)-C(7 C)$ & $1.380(6)$ & $C(14 B)-C(12 B)-C(13 B)$ & $109.8(4)$ \\
\hline$C(1 D)-C(2 D)$ & $1.498(6)$ & $N(4 A)-C(16 A)-N(3 A)$ & $115.6(4)$ \\
\hline$C(2 D)-C(7 D)$ & $1.382(6)$ & $N(4 A)-C(16 A)-C(17 A)$ & $121.3(4)$ \\
\hline$C(2 D)-C(3 D)$ & $1.387(6)$ & $N(3 A)-C(16 A)-C(17 A)$ & $123.1(4)$ \\
\hline$C(3 D)-C(4 D)$ & $1.389(6)$ & $C(18 A)-C(17 A)-C(16 A)$ & $119.1(5)$ \\
\hline$C(4 D)-C(5 D)$ & $1.369(7)$ & $\mathrm{N}(4 \mathrm{~B})-\mathrm{C}(16 \mathrm{~B})-\mathrm{N}(3 \mathrm{~B})$ & $116.2(4)$ \\
\hline$C(5 D)-C(6 D)$ & $1.379(7)$ & $N(4 B)-C(16 B)-C(17 B)$ & $120.2(4)$ \\
\hline$C(6 D)-C(7 D)$ & $1.376(6)$ & $N(3 B)-C(16 B)-C(17 B)$ & $123.6(4)$ \\
\hline$C(1 E)-C(2 E)$ & $1.528(12)$ & $C(17 A)-C(18 A)-C(19 A)$ & $119.6(5)$ \\
\hline$C(2 E)-C(3 E)$ & $1.378(11)$ & $C(18 B)-C(17 B)-C(16 B)$ & $118.9(5)$ \\
\hline$C(2 E)-C(7 E)$ & $1.379(11)$ & $C(20 A)-C(19 A)-C(18 A)$ & $118.6(5)$ \\
\hline$C(3 E)-C(4 E)$ & $1.370(11)$ & $C(19 B)-C(18 B)-C(17 B)$ & $120.1(4)$ \\
\hline$C(4 E)-C(5 E)$ & $1.372(10)$ & $N(4 A)-C(20 A)-C(19 A)$ & $122.1(5)$ \\
\hline$C(5 E)-C(6 E)$ & $1.385(12)$ & $C(20 B)-C(19 B)-C(18 B)$ & $118.3(5)$ \\
\hline$C(6 E)-C(7 E)$ & $1.380(12)$ & $C(26 A)-C(21 A)-C(22 A)$ & $120.6(4)$ \\
\hline$C(1 F)-C(2 F)$ & $1.521(14)$ & $C(26 A)-C(21 A)-N(3 A)$ & $121.6(4)$ \\
\hline$C(2 F)-C(3 F)$ & $1.380(13)$ & $C(22 A)-C(21 A)-N(3 A)$ & $117.8(4)$ \\
\hline$C(2 F)-C(7 F)$ & $1.387(13)$ & $N(4 B)-C(20 B)-C(19 B)$ & $122.8(5)$ \\
\hline$C(3 F)-C(4 F)$ & $1.379(13)$ & $C(21 A)-C(22 A)-C(23 A)$ & $117.9(5)$ \\
\hline$C(4 F)-C(5 F)$ & $1.371(13)$ & $C(21 A)-C(22 A)-C(27 A)$ & $121.0(4)$ \\
\hline$C(5 F)-C(6 F)$ & $1.379(13)$ & $C(23 A)-C(22 A)-C(27 A)$ & $121.2(5)$ \\
\hline \multirow[t]{2}{*}{$C(6 F)-C(7 F)$} & $1.384(13)$ & $C(26 B)-C(21 B)-C(22 B)$ & $121.9(4)$ \\
\hline & & $C(26 B)-C(21 B)-N(3 B)$ & $121.1(4)$ \\
\hline $\mathrm{Cl}(1)-\mathrm{Cu}(1)-\mathrm{Cu}(3)$ & $139.59(4)$ & $C(22 B)-C(21 B)-N(3 B)$ & $116.8(4)$ \\
\hline $\mathrm{Cl}(1)-\mathrm{Cu}(1)-\mathrm{Cu}(2)$ & $141.07(4)$ & $C(24 A)-C(23 A)-C(22 A)$ & $122.5(5)$ \\
\hline $\mathrm{Cu}(3)-\mathrm{Cu}(1)-\mathrm{Cu}(2)$ & $61.98(2)$ & $C(23 B)-C(22 B)-C(21 B)$ & $118.4(5)$ \\
\hline $\mathrm{Cl}(1)-\mathrm{Cu}(1)-\mathrm{Si}(1 \mathrm{~B})$ & $97.22(5)$ & $C(23 B)-C(22 B)-C(27 B)$ & $119.4(4)$ \\
\hline $\mathrm{Cu}(3)-\mathrm{Cu}(1)-\mathrm{Si}(1 \mathrm{~B})$ & $54.12(4)$ & $C(21 B)-C(22 B)-C(27 B)$ & $122.2(4)$ \\
\hline $\mathrm{Cu}(2)-\mathrm{Cu}(1)-\mathrm{Si}(1 \mathrm{~B})$ & $115.46(4)$ & $C(23 A)-C(24 A)-C(25 A)$ & $117.9(5)$ \\
\hline $\mathrm{Cl}(1)-\mathrm{Cu}(1)-\mathrm{Si}(1 \mathrm{~A})$ & $98.34(5)$ & $C(23 A)-C(24 A)-C(28 A)$ & $120.4(6)$ \\
\hline $\mathrm{Cu}(3)-\mathrm{Cu}(1)-\mathrm{Si}(1 \mathrm{~A})$ & $115.29(4)$ & $C(25 A)-C(24 A)-C(28 A)$ & $121.7(6)$ \\
\hline $\mathrm{Cu}(2)-\mathrm{Cu}(1)-\mathrm{Si}(1 \mathrm{~A})$ & $53.63(4)$ & $C(22 B)-C(23 B)-C(24 B)$ & $121.2(5)$ \\
\hline $\mathrm{Si}(1 \mathrm{~B})-\mathrm{Cu}(1)-\mathrm{Si}(1 \mathrm{~A})$ & $162.85(4)$ & $C(24 A)-C(25 A)-C(26 A)$ & $122.4(5)$ \\
\hline$N(4 A)-C u(2)-S i(1 A)$ & $85.94(12)$ & $C(23 B)-C(24 B)-C(25 B)$ & $119.4(5)$ \\
\hline $\mathrm{N}(4 \mathrm{~A})-\mathrm{Cu}(2)-\mathrm{Cl}(2)$ & $100.48(12)$ & $C(23 B)-C(24 B)-C(28 B)$ & $119.2(5)$ \\
\hline $\mathrm{Si}(1 \mathrm{~A})-\mathrm{Cu}(2)-\mathrm{Cl}(2)$ & $129.69(5)$ & $C(25 B)-C(24 B)-C(28 B)$ & $121.4(6)$ \\
\hline $\mathrm{N}(4 \mathrm{~A})-\mathrm{Cu}(2)-\mathrm{Cl}(3)$ & $107.78(12)$ & $C(21 A)-C(26 A)-C(25 A)$ & $118.7(5)$ \\
\hline $\mathrm{Si}(1 \mathrm{~A})-\mathrm{Cu}(2)-\mathrm{Cl}(3)$ & $129.27(5)$ & $C(21 A)-C(26 A)-C(29 A)$ & $120.3(4)$ \\
\hline $\mathrm{Cl}(2)-\mathrm{Cu}(2)-\mathrm{Cl}(3)$ & $96.42(5)$ & $C(25 A)-C(26 A)-C(29 A)$ & $120.9(5)$ \\
\hline $\mathrm{N}(4 \mathrm{~A})-\mathrm{Cu}(2)-\mathrm{Cu}(1)$ & $151.59(12)$ & $C(24 B)-C(25 B)-C(26 B)$ & $121.7(5)$ \\
\hline $\mathrm{Si}(1 \mathrm{~A})-\mathrm{Cu}(2)-\mathrm{Cu}(1)$ & $65.84(4)$ & $C(21 B)-C(26 B)-C(25 B)$ & $117.4(5)$ \\
\hline $\mathrm{Cl}(2)-\mathrm{Cu}(2)-\mathrm{Cu}(1)$ & $100.18(4)$ & $C(21 B)-C(26 B)-C(29 B)$ & $121.7(5)$ \\
\hline $\mathrm{Cl}(3)-\mathrm{Cu}(2)-\mathrm{Cu}(1)$ & $89.00(4)$ & $C(25 B)-C(26 B)-C(29 B)$ & $120.9(5)$ \\
\hline
\end{tabular}




\begin{tabular}{|c|c|c|c|}
\hline $\mathrm{N}(4 \mathrm{~A})-\mathrm{Cu}(2)-\mathrm{Cu}(3)$ & $149.59(12)$ & $C(5 A)-C(4 A)-C(3 A)$ & 119.1(5) \\
\hline $\mathrm{Si}(1 \mathrm{~A})-\mathrm{Cu}(2)-\mathrm{Cu}(3)$ & $124.27(5)$ & $C(3 C)-C(2 C)-C(7 C)$ & $118.3(4)$ \\
\hline $\mathrm{Cl}(2)-\mathrm{Cu}(2)-\mathrm{Cu}(3)$ & $59.09(4)$ & $C(3 C)-C(2 C)-C(1 C)$ & $123.0(5)$ \\
\hline $\mathrm{Cl}(3)-\mathrm{Cu}(2)-\mathrm{Cu}(3)$ & $57.85(4)$ & $C(7 C)-C(2 C)-C(1 C)$ & $118.6(5)$ \\
\hline $\mathrm{Cu}(1)-\mathrm{Cu}(2)-\mathrm{Cu}(3)$ & $58.82(3)$ & $C(4 C)-C(3 C)-C(2 C)$ & $121.0(4)$ \\
\hline $\mathrm{Cu}(2)-\mathrm{Cl}(2)-\mathrm{Cu}(3)$ & $63.31(3)$ & $C(5 C)-C(4 C)-C(3 C)$ & $120.1(4)$ \\
\hline $\mathrm{N}(4 \mathrm{~B})-\mathrm{Cu}(3)-\mathrm{Si}(1 \mathrm{~B})$ & $85.82(12)$ & $C(6 C)-C(5 C)-C(4 C)$ & $119.7(4)$ \\
\hline $\mathrm{N}(4 \mathrm{~B})-\mathrm{Cu}(3)-\mathrm{Cl}(3)$ & $107.17(12)$ & $C(5 C)-C(6 C)-C(7 C)$ & $120.3(4)$ \\
\hline $\mathrm{Si}(1 \mathrm{~B})-\mathrm{Cu}(3)-\mathrm{Cl}(3)$ & $131.00(5)$ & $C(6 C)-C(7 C)-C(2 C)$ & $120.5(4)$ \\
\hline $\mathrm{N}(4 \mathrm{~B})-\mathrm{Cu}(3)-\mathrm{Cl}(2)$ & $101.34(12)$ & $C(7 D)-C(2 D)-C(3 D)$ & $118.0(4)$ \\
\hline $\mathrm{Si}(1 \mathrm{~B})-\mathrm{Cu}(3)-\mathrm{Cl}(2)$ & $127.73(5)$ & $C(7 D)-C(2 D)-C(1 D)$ & $120.0(4)$ \\
\hline $\mathrm{Cl}(3)-\mathrm{Cu}(3)-\mathrm{Cl}(2)$ & $96.55(5)$ & $C(3 D)-C(2 D)-C(1 D)$ & $122.0(4)$ \\
\hline $\mathrm{N}(4 \mathrm{~B})-\mathrm{Cu}(3)-\mathrm{Cu}(1)$ & $151.04(12)$ & $C(2 D)-C(3 D)-C(4 D)$ & $121.4(4)$ \\
\hline $\mathrm{Si}(1 \mathrm{~B})-\mathrm{Cu}(3)-\mathrm{Cu}(1)$ & $65.47(4)$ & $C(5 D)-C(4 D)-C(3 D)$ & $119.9(4)$ \\
\hline $\mathrm{Cl}(3)-\mathrm{Cu}(3)-\mathrm{Cu}(1)$ & $90.22(4)$ & $C(4 D)-C(5 D)-C(6 D)$ & $118.8(4)$ \\
\hline $\mathrm{Cl}(2)-\mathrm{Cu}(3)-\mathrm{Cu}(1)$ & $99.38(4)$ & $C(7 D)-C(6 D)-C(5 D)$ & $121.7(5)$ \\
\hline $\mathrm{N}(4 \mathrm{~B})-\mathrm{Cu}(3)-\mathrm{Cu}(2)$ & $149.74(12)$ & $C(6 D)-C(7 D)-C(2 D)$ & $120.2(4)$ \\
\hline $\mathrm{Si}(1 \mathrm{~B})-\mathrm{Cu}(3)-\mathrm{Cu}(2)$ & $123.90(5)$ & $C(3 E)-C(2 E)-C(7 E)$ & 118.3(10) \\
\hline $\mathrm{Cl}(3)-\mathrm{Cu}(3)-\mathrm{Cu}(2)$ & $59.51(4)$ & $C(3 E)-C(2 E)-C(1 E)$ & $116.2(10)$ \\
\hline $\mathrm{Cl}(2)-\mathrm{Cu}(3)-\mathrm{Cu}(2)$ & $57.60(4)$ & $C(7 E)-C(2 E)-C(1 E)$ & $125.5(11)$ \\
\hline $\mathrm{Cu}(1)-\mathrm{Cu}(3)-\mathrm{Cu}(2)$ & $59.19(3)$ & $C(4 E)-C(3 E)-C(2 E)$ & $122.5(10)$ \\
\hline $\mathrm{Cu}(3)-\mathrm{Cl}(3)-\mathrm{Cu}(2)$ & $62.64(3)$ & $C(3 E)-C(4 E)-C(5 E)$ & $119.8(10)$ \\
\hline$N(2 A)-S i(1 A)-N(3 A)$ & $104.78(18)$ & $C(4 E)-C(5 E)-C(6 E)$ & $118.0(11)$ \\
\hline$N(2 A)-S i(1 A)-N(1 A)$ & $71.38(17)$ & $C(7 E)-C(6 E)-C(5 E)$ & $122.2(11)$ \\
\hline$N(3 A)-S i(1 A)-N(1 A)$ & $100.32(17)$ & $C(2 E)-C(7 E)-C(6 E)$ & 119.2(11) \\
\hline$N(2 A)-S i(1 A)-C u(2)$ & $121.03(13)$ & $C(3 F)-C(2 F)-C(7 F)$ & $118.9(13)$ \\
\hline$N(3 A)-S i(1 A)-C u(2)$ & $92.08(12)$ & $C(3 F)-C(2 F)-C(1 F)$ & $122.3(15)$ \\
\hline$N(1 A)-S i(1 A)-C u(2)$ & $159.67(14)$ & $C(7 F)-C(2 F)-C(1 F)$ & $118.8(15)$ \\
\hline$N(2 A)-S i(1 A)-C(1 A)$ & $35.70(17)$ & $C(4 F)-C(3 F)-C(2 F)$ & 121.1(16) \\
\hline$N(3 A)-S i(1 A)-C(1 A)$ & $108.99(17)$ & $C(5 F)-C(4 F)-C(3 F)$ & $118.3(16)$ \\
\hline$C(6 F)-C(7 F)-C(2 F)$ & $121.3(16)$ & $C(4 F)-C(5 F)-C(6 F)$ & $122.8(15)$ \\
\hline$C(5 F)-C(6 F)-C(7 F)$ & $117.5(16)$ & & \\
\hline
\end{tabular}




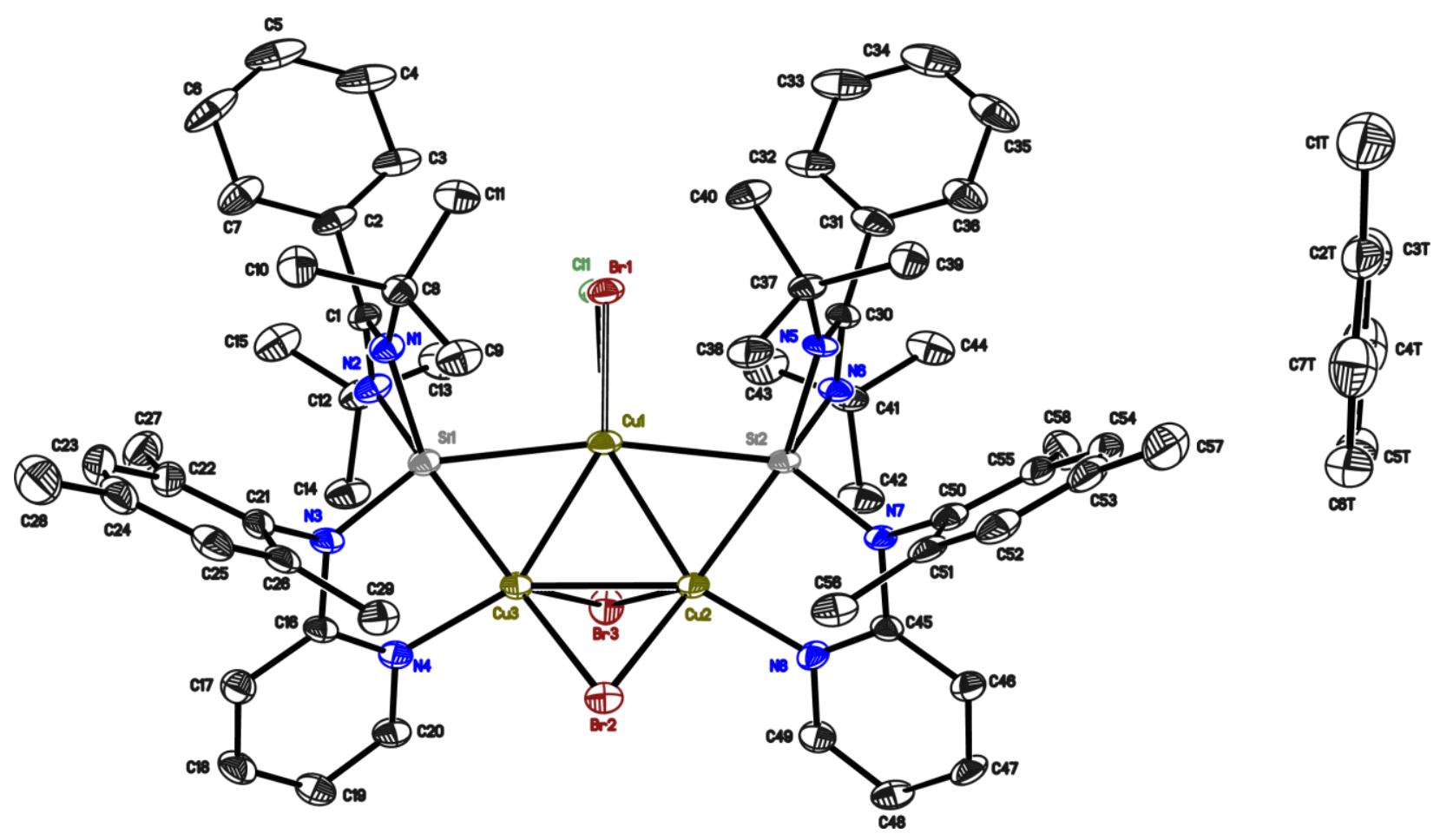

The structure crystallizes as pseudo-merohedral twin with the twin law -1.000 .020 .00$ $-0.011 .00-0.010 .000 .05-1.00$. The fractional contribution of the minor component refines to $0.2603(10)$.

One bromide atom $(\mathrm{Br} 1 / \mathrm{Cl} 1)$ is disordered with a chloride atom. The anisotropic displacement parameters are constrained to be identical. The occupancy of the chloride atom refines to $0.431(4)$

Partially bromide-chloride-disorder is supposed to stem from a halide-interchange with the chloro benzamidinato silylene NHSiCl 1.

Although all necessary disordered toluene molecules were carefully refined with all possible restraints (see Table S2.2, left), only a SQUEEZE-refinement in the region of the two toluene molecules (C1U-C7U; C1W-C7W/C1X-C7X; see Table S2.2, right) was able to eliminate the reported A-Alerts (see Table S2.2) and lead to an improved model quality. 
Table S2.2: Comparison of the refinement of $\mathbf{3 b}$ without (left) und with (right) SQUEEZE.

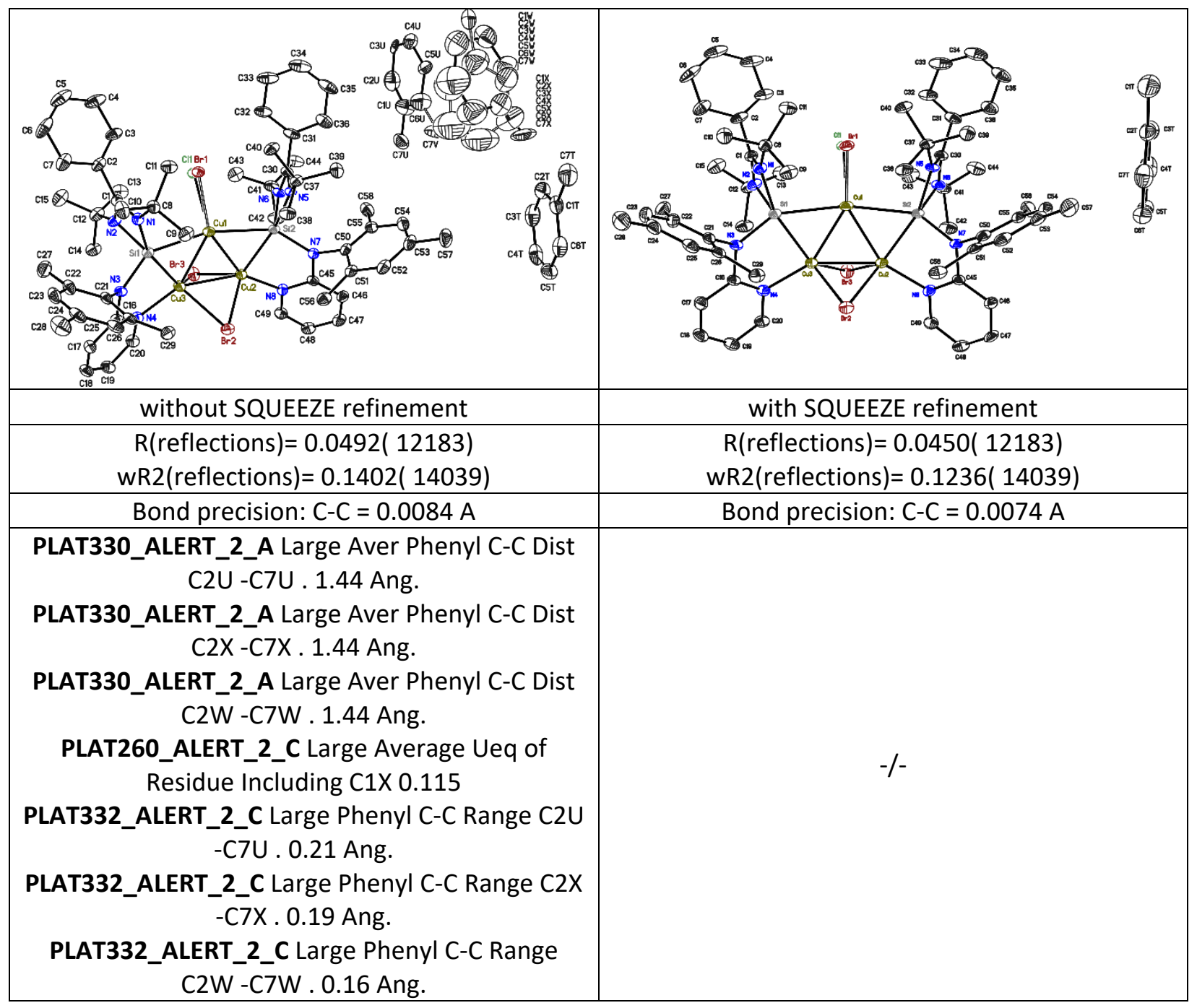

Bond lengths $[\AA \AA]$ and angles $\left[^{\circ}\right]$ for $3 b$ :

\begin{tabular}{|l|l|l|l|}
\hline $\mathrm{Si}(1)-\mathrm{N}(3)$ & $1.823(4)$ & $\mathrm{Si}(2)-\mathrm{Cu}(2)-\mathrm{Br}(3)$ & $128.12(4)$ \\
\hline $\mathrm{Si}(1)-\mathrm{N}(2)$ & $1.839(4)$ & $\mathrm{Cu}(1)-\mathrm{Cu}(2)-\mathrm{Br}(3)$ & $88.17(3)$ \\
\hline $\mathrm{Si}(1)-\mathrm{N}(1)$ & $1.853(4)$ & $\mathrm{Br}(2)-\mathrm{Cu}(2)-\mathrm{Br}(3)$ & $100.48(3)$ \\
\hline $\mathrm{Si}(1)-\mathrm{Cu}(3)$ & $2.2689(15)$ & $\mathrm{N}(8)-\mathrm{Cu}(2)-\mathrm{Cu}(3)$ & $149.39(12)$ \\
\hline $\mathrm{Si}(1)-\mathrm{C}(1)$ & $2.286(5)$ & $\mathrm{Si}(2)-\mathrm{Cu}(2)-\mathrm{Cu}(3)$ & $124.59(5)$ \\
\hline $\mathrm{Si}(1)-\mathrm{Cu}(1)$ & $2.5812(14)$ & $\mathrm{Cu}(1)-\mathrm{Cu}(2)-\mathrm{Cu}(3)$ & $58.63(3)$ \\
\hline $\mathrm{Si}(2)-\mathrm{N}(7)$ & $1.814(4)$ & $\mathrm{Br}(2)-\mathrm{Cu}(2)-\mathrm{Cu}(3)$ & $59.83(3)$ \\
\hline $\mathrm{Si}(2)-\mathrm{N}(6)$ & $1.839(4)$ & $\mathrm{Br}(3)-\mathrm{Cu}(2)-\mathrm{Cu}(3)$ & $59.67(3)$ \\
\hline $\mathrm{Si}(2)-\mathrm{N}(5)$ & $1.853(4)$ & $\mathrm{N}(4)-\mathrm{Cu}(3)-\mathrm{Si}(1)$ & $85.39(11)$ \\
\hline $\mathrm{Si}(2)-\mathrm{Cu}(2)$ & $2.2678(15)$ & $\mathrm{N}(4)-\mathrm{Cu}(3)-\mathrm{Cu}(1)$ & $151.49(11)$ \\
\hline
\end{tabular}




\begin{tabular}{|c|c|c|c|}
\hline $\mathrm{Si}(2)-\mathrm{C}(30)$ & $2.299(5)$ & $\mathrm{Si}(1)-\mathrm{Cu}(3)-\mathrm{Cu}(1)$ & $66.50(4)$ \\
\hline $\mathrm{Si}(2)-\mathrm{Cu}(1)$ & $2.5806(14)$ & $\mathrm{N}(4)-\mathrm{Cu}(3)-\mathrm{Br}(2)$ & $100.87(11)$ \\
\hline $\mathrm{Cu}(1)-\mathrm{Br}(1)$ & $2.307(5)$ & $\mathrm{Si}(1)-\mathrm{Cu}(3)-\mathrm{Br}(2)$ & $125.89(4)$ \\
\hline $\mathrm{Cu}(1)-\mathrm{Cl}(1)$ & $2.384(18)$ & $\mathrm{Cu}(1)-\mathrm{Cu}(3)-\mathrm{Br}(2)$ & $99.59(3)$ \\
\hline $\mathrm{Cu}(1)-\mathrm{Cu}(2)$ & $2.4289(11)$ & $\mathrm{N}(4)-\mathrm{Cu}(3)-\mathrm{Br}(3)$ & $107.10(11)$ \\
\hline $\mathrm{Cu}(1)-\mathrm{Cu}(3)$ & $2.4323(11)$ & $\mathrm{Si}(1)-\mathrm{Cu}(3)-\mathrm{Br}(3)$ & $129.13(4)$ \\
\hline $\mathrm{Cu}(2)-\mathrm{N}(8)$ & $2.005(4)$ & $\mathrm{Cu}(1)-\mathrm{Cu}(3)-\mathrm{Br}(3)$ & $88.33(3)$ \\
\hline $\mathrm{Cu}(2)-\mathrm{Br}(2)$ & $2.5043(10)$ & $\mathrm{Br}(2)-\mathrm{Cu}(3)-\mathrm{Br}(3)$ & $100.53(3)$ \\
\hline $\mathrm{Cu}(2)-\mathrm{Br}(3)$ & $2.5308(9)$ & $\mathrm{N}(4)-\mathrm{Cu}(3)-\mathrm{Cu}(2)$ & $150.01(11)$ \\
\hline $\mathrm{Cu}(2)-\mathrm{Cu}(3)$ & $2.5350(9)$ & $\mathrm{Si}(1)-\mathrm{Cu}(3)-\mathrm{Cu}(2)$ & $124.18(5)$ \\
\hline $\mathrm{Cu}(3)-\mathrm{N}(4)$ & $2.014(4)$ & $\mathrm{Cu}(1)-\mathrm{Cu}(3)-\mathrm{Cu}(2)$ & $58.50(3)$ \\
\hline $\mathrm{Cu}(3)-\mathrm{Br}(2)$ & $2.5132(10)$ & $\mathrm{Br}(2)-\mathrm{Cu}(3)-\mathrm{Cu}(2)$ & $59.48(3)$ \\
\hline $\mathrm{Cu}(3)-\mathrm{Br}(3)$ & $2.5202(9)$ & $\mathrm{Br}(3)-\mathrm{Cu}(3)-\mathrm{Cu}(2)$ & $60.08(3)$ \\
\hline$C(1)-N(1)$ & $1.327(6)$ & $\mathrm{Cu}(2)-\mathrm{Br}(2)-\mathrm{Cu}(3)$ & $60.69(2)$ \\
\hline $\mathrm{C}(1)-\mathrm{N}(2)$ & $1.337(6)$ & $\mathrm{Cu}(3)-\mathrm{Br}(3)-\mathrm{Cu}(2)$ & $60.25(2)$ \\
\hline$C(1)-C(2)$ & $1.503(6)$ & $N(1)-C(1)-N(2)$ & $107.3(4)$ \\
\hline$N(1)-C(8)$ & $1.509(6)$ & $N(1)-C(1)-C(2)$ & $127.8(4)$ \\
\hline$C(2)-C(7)$ & $1.387(7)$ & $N(2)-C(1)-C(2)$ & $124.9(4)$ \\
\hline$C(2)-C(3)$ & $1.392(8)$ & $\mathrm{N}(1)-\mathrm{C}(1)-\mathrm{Si}(1)$ & $54.1(2)$ \\
\hline$N(2)-C(12)$ & $1.495(6)$ & $\mathrm{N}(2)-\mathrm{C}(1)-\mathrm{Si}(1)$ & $53.6(2)$ \\
\hline$C(3)-C(4)$ & $1.397(7)$ & $C(2)-C(1)-S i(1)$ & $174.4(3)$ \\
\hline$N(3)-C(16)$ & $1.397(6)$ & $C(1)-N(1)-C(8)$ & 129.1(4) \\
\hline$N(3)-C(21)$ & $1.442(6)$ & $\mathrm{C}(1)-\mathrm{N}(1)-\mathrm{Si}(1)$ & $90.4(3)$ \\
\hline$N(7)-C(45)$ & $1.391(6)$ & $\mathrm{C}(8)-\mathrm{N}(1)-\mathrm{Si}(1)$ & $138.4(3)$ \\
\hline$N(7)-C(50)$ & $1.447(6)$ & $C(7)-C(2)-C(3)$ & $120.5(5)$ \\
\hline$C(7)-C(6)$ & $1.410(8)$ & $C(7)-C(2)-C(1)$ & $118.8(5)$ \\
\hline$N(6)-C(30)$ & $1.342(6)$ & $C(3)-C(2)-C(1)$ & $120.5(4)$ \\
\hline$N(6)-C(41)$ & $1.485(6)$ & $C(1)-N(2)-C(12)$ & $131.7(4)$ \\
\hline$C(6)-C(5)$ & $1.385(10)$ & $C(1)-N(2)-S i(1)$ & $90.6(3)$ \\
\hline$N(5)-C(30)$ & $1.343(6)$ & $C(12)-N(2)-S i(1)$ & $136.9(3)$ \\
\hline$N(5)-C(37)$ & $1.499(6)$ & $C(2)-C(3)-C(4)$ & $119.7(5)$ \\
\hline$C(5)-C(4)$ & $1.365(10)$ & $C(16)-N(3)-C(21)$ & $115.5(4)$ \\
\hline$N(4)-C(16)$ & $1.338(6)$ & $C(16)-N(3)-S i(1)$ & $118.9(3)$ \\
\hline$N(4)-C(20)$ & $1.347(6)$ & $C(21)-N(3)-S i(1)$ & $125.1(3)$ \\
\hline$N(8)-C(45)$ & $1.343(6)$ & $C(45)-N(7)-C(50)$ & $115.4(3)$ \\
\hline$N(8)-C(49)$ & $1.351(6)$ & $C(45)-N(7)-S i(2)$ & $119.6(3)$ \\
\hline$C(8)-C(9)$ & $1.520(6)$ & $C(50)-N(7)-\operatorname{Si}(2)$ & $124.6(3)$ \\
\hline$C(8)-C(11)$ & $1.523(7)$ & $C(2)-C(7)-C(6)$ & 119.1(6) \\
\hline$C(8)-C(10)$ & $1.529(6)$ & $C(30)-N(6)-C(41)$ & $131.4(4)$ \\
\hline$C(12)-C(13)$ & $1.509(8)$ & $C(30)-N(6)-\operatorname{Si}(2)$ & $91.2(3)$ \\
\hline$C(12)-C(14)$ & $1.531(7)$ & $C(41)-N(6)-\operatorname{Si}(2)$ & $136.5(3)$ \\
\hline$C(12)-C(15)$ & $1.532(7)$ & $C(5)-C(6)-C(7)$ & $119.7(5)$ \\
\hline$C(16)-C(17)$ & $1.412(7)$ & $C(30)-N(5)-C(37)$ & $129.0(4)$ \\
\hline$C(19)-C(20)$ & $1.373(7)$ & $C(30)-N(5)-S i(2)$ & $90.5(3)$ \\
\hline
\end{tabular}




\begin{tabular}{|c|c|c|c|}
\hline$C(19)-C(18)$ & \begin{tabular}{|l|}
$1.389(7)$ \\
\end{tabular} & $C(37)-N(5)-\operatorname{Si}(2)$ & $138.4(3)$ \\
\hline$C(18)-C(17)$ & $1.383(7)$ & $C(4)-C(5)-C(6)$ & $121.0(5)$ \\
\hline$C(21)-C(22)$ & $1.401(6)$ & $C(16)-N(4)-C(20)$ & $119.7(4)$ \\
\hline$C(21)-C(26)$ & $1.406(7)$ & $\mathrm{C}(16)-\mathrm{N}(4)-\mathrm{Cu}(3)$ & $117.0(3)$ \\
\hline$C(22)-C(23)$ & $1.398(7)$ & $\mathrm{C}(20)-\mathrm{N}(4)-\mathrm{Cu}(3)$ & $122.2(3)$ \\
\hline$C(22)-C(27)$ & $1.501(7)$ & $C(5)-C(4)-C(3)$ & $120.0(6)$ \\
\hline$C(23)-C(24)$ & $1.392(8)$ & $\mathrm{C}(45)-\mathrm{N}(8)-\mathrm{C}(49)$ & $119.1(4)$ \\
\hline$C(24)-C(25)$ & $1.382(7)$ & $\mathrm{C}(45)-\mathrm{N}(8)-\mathrm{Cu}(2)$ & 117.1(3) \\
\hline$C(24)-C(28)$ & $1.510(7)$ & $\mathrm{C}(49)-\mathrm{N}(8)-\mathrm{Cu}(2)$ & $122.4(3)$ \\
\hline$C(25)-C(26)$ & $1.391(7)$ & $\mathrm{N}(1)-\mathrm{C}(8)-\mathrm{C}(9)$ & $105.7(4)$ \\
\hline$C(26)-C(29)$ & $1.512(6)$ & $N(1)-C(8)-C(11)$ & $111.8(4)$ \\
\hline$C(30)-C(31)$ & $1.495(7)$ & $C(9)-C(8)-C(11)$ & $109.1(4)$ \\
\hline$C(31)-C(36)$ & $1.377(8)$ & $\mathrm{N}(1)-\mathrm{C}(8)-\mathrm{C}(10)$ & $109.8(4)$ \\
\hline$C(31)-C(32)$ & $1.403(8)$ & $C(9)-C(8)-C(10)$ & $110.1(4)$ \\
\hline$C(32)-C(33)$ & $1.382(8)$ & $C(11)-C(8)-C(10)$ & $110.2(4)$ \\
\hline$C(33)-C(34)$ & $1.360(10)$ & $N(2)-C(12)-C(13)$ & $110.1(4)$ \\
\hline$C(34)-C(35)$ & $1.374(10)$ & $N(2)-C(12)-C(14)$ & $105.5(4)$ \\
\hline$C(35)-C(36)$ & $1.413(8)$ & $C(13)-C(12)-C(14)$ & $109.5(4)$ \\
\hline$C(37)-C(38)$ & $1.514(7)$ & $N(2)-C(12)-C(15)$ & $111.3(4)$ \\
\hline$C(37)-C(40)$ & $1.530(6)$ & $C(13)-C(12)-C(15)$ & $110.9(5)$ \\
\hline$C(37)-C(39)$ & $1.530(6)$ & $C(14)-C(12)-C(15)$ & $109.4(4)$ \\
\hline$C(41)-C(44)$ & $1.526(7)$ & $N(4)-C(16)-N(3)$ & $116.2(4)$ \\
\hline$C(41)-C(43)$ & $1.532(7)$ & $N(4)-C(16)-C(17)$ & $120.8(4)$ \\
\hline$C(41)-C(42)$ & $1.538(7)$ & $N(3)-C(16)-C(17)$ & $122.9(4)$ \\
\hline$C(45)-C(46)$ & $1.411(6)$ & $C(20)-C(19)-C(18)$ & $118.5(5)$ \\
\hline$C(46)-C(47)$ & $1.384(7)$ & $C(17)-C(18)-C(19)$ & $119.7(5)$ \\
\hline$C(47)-C(48)$ & $1.392(7)$ & $C(18)-C(17)-C(16)$ & $118.6(4)$ \\
\hline$C(48)-C(49)$ & $1.374(7)$ & $N(4)-C(20)-C(19)$ & $122.5(4)$ \\
\hline$C(50)-C(51)$ & $1.404(7)$ & $C(22)-C(21)-C(26)$ & $120.7(4)$ \\
\hline$C(50)-C(55)$ & $1.404(7)$ & $C(22)-C(21)-N(3)$ & $121.3(4)$ \\
\hline$C(51)-C(52)$ & $1.406(7)$ & $C(26)-C(21)-N(3)$ & $117.9(4)$ \\
\hline$C(51)-C(56)$ & $1.505(7)$ & $C(23)-C(22)-C(21)$ & $118.6(5)$ \\
\hline$C(52)-C(53)$ & $1.389(8)$ & $C(23)-C(22)-C(27)$ & $120.2(4)$ \\
\hline$C(53)-C(54)$ & $1.391(8)$ & $C(21)-C(22)-C(27)$ & $121.2(4)$ \\
\hline$C(53)-C(57)$ & $1.519(7)$ & $C(24)-C(23)-C(22)$ & $121.6(4)$ \\
\hline$C(54)-C(55)$ & $1.400(7)$ & $C(25)-C(24)-C(23)$ & $118.4(5)$ \\
\hline$C(55)-C(58)$ & $1.499(7)$ & $C(25)-C(24)-C(28)$ & $120.1(5)$ \\
\hline$C(1 \mathrm{~T})-\mathrm{C}(2 \mathrm{~T})$ & $1.516(7)$ & $C(23)-C(24)-C(28)$ & $121.5(5)$ \\
\hline $\mathrm{C}(2 \mathrm{~T})-\mathrm{C}(3 \mathrm{~T})$ & $1.375(7)$ & $C(24)-C(25)-C(26)$ & $122.2(5)$ \\
\hline$C(2 T)-C(7 T)$ & $1.403(7)$ & $C(25)-C(26)-C(21)$ & $118.4(4)$ \\
\hline $\mathrm{C}(3 \mathrm{~T})-\mathrm{C}(4 \mathrm{~T})$ & $1.376(8)$ & $C(25)-C(26)-C(29)$ & $120.4(4)$ \\
\hline $\mathrm{C}(4 \mathrm{~T})-\mathrm{C}(5 \mathrm{~T})$ & $1.380(8)$ & $C(21)-C(26)-C(29)$ & $121.1(4)$ \\
\hline$C(5 T)-C(6 T)$ & $1.370(8)$ & $N(6)-C(30)-N(5)$ & $106.3(4)$ \\
\hline $\mathrm{C}(6 \mathrm{~T})-\mathrm{C}(7 \mathrm{~T})$ & $1.403(7)$ & $N(6)-C(30)-C(31)$ & $125.1(4)$ \\
\hline
\end{tabular}




\begin{tabular}{|c|c|c|c|}
\hline & & $N(5)-C(30)-C(31)$ & $128.6(4)$ \\
\hline$N(3)-S i(1)-N(2)$ & $104.24(18)$ & $N(6)-C(30)-S i(2)$ & $53.1(2)$ \\
\hline$N(3)-S i(1)-N(1)$ & $100.97(17)$ & $N(5)-C(30)-S i(2)$ & $53.7(2)$ \\
\hline$N(2)-S i(1)-N(1)$ & $71.03(17)$ & $C(31)-C(30)-S i(2)$ & $174.7(4)$ \\
\hline $\mathrm{N}(3)-\mathrm{Si}(1)-\mathrm{Cu}(3)$ & $91.63(13)$ & $C(36)-C(31)-C(32)$ & $119.9(5)$ \\
\hline $\mathrm{N}(2)-\mathrm{Si}(1)-\mathrm{Cu}(3)$ & $121.81(13)$ & $C(36)-C(31)-C(30)$ & $119.4(5)$ \\
\hline $\mathrm{N}(1)-\mathrm{Si}(1)-\mathrm{Cu}(3)$ & $159.34(13)$ & $C(32)-C(31)-C(30)$ & $120.5(4)$ \\
\hline$N(3)-S i(1)-C(1)$ & $108.60(17)$ & $C(33)-C(32)-C(31)$ & $119.5(5)$ \\
\hline$N(2)-S i(1)-C(1)$ & $35.78(17)$ & $C(34)-C(33)-C(32)$ & $120.7(6)$ \\
\hline$N(1)-S i(1)-C(1)$ & $35.48(17)$ & $C(33)-C(34)-C(35)$ & $121.0(5)$ \\
\hline $\mathrm{Cu}(3)-\mathrm{Si}(1)-\mathrm{C}(1)$ & $152.15(13)$ & $C(34)-C(35)-C(36)$ & $119.4(5)$ \\
\hline $\mathrm{N}(3)-\mathrm{Si}(1)-\mathrm{Cu}(1)$ & $145.52(13)$ & $C(31)-C(36)-C(35)$ & $119.5(6)$ \\
\hline$N(2)-S i(1)-C u(1)$ & $107.25(13)$ & $N(5)-C(37)-C(38)$ & $106.8(4)$ \\
\hline $\mathrm{N}(1)-\mathrm{Si}(1)-\mathrm{Cu}(1)$ & $102.21(13)$ & $N(5)-C(37)-C(40)$ & $111.8(4)$ \\
\hline $\mathrm{Cu}(3)-\mathrm{Si}(1)-\mathrm{Cu}(1)$ & $59.78(4)$ & $C(38)-C(37)-C(40)$ & $108.9(4)$ \\
\hline$C(1)-S i(1)-C u(1)$ & $105.11(12)$ & $N(5)-C(37)-C(39)$ & $109.8(3)$ \\
\hline$N(7)-S i(2)-N(6)$ & $104.15(17)$ & $C(38)-C(37)-C(39)$ & $109.6(4)$ \\
\hline$N(7)-S i(2)-N(5)$ & $101.07(17)$ & $C(40)-C(37)-C(39)$ & $110.0(4)$ \\
\hline$N(6)-\operatorname{Si}(2)-N(5)$ & $71.18(17)$ & $N(6)-C(41)-C(44)$ & $111.8(4)$ \\
\hline $\mathrm{N}(7)-\mathrm{Si}(2)-\mathrm{Cu}(2)$ & 91.91(13) & $N(6)-C(41)-C(43)$ & $110.4(4)$ \\
\hline$N(6)-\operatorname{Si}(2)-C u(2)$ & $121.24(13)$ & $C(44)-C(41)-C(43)$ & $110.7(4)$ \\
\hline$N(5)-S i(2)-C u(2)$ & 159.40(13) & $N(6)-C(41)-C(42)$ & $105.8(4)$ \\
\hline$N(7)-S i(2)-C(30)$ & $108.85(17)$ & $C(44)-C(41)-C(42)$ & $109.0(4)$ \\
\hline$N(6)-S i(2)-C(30)$ & $35.70(17)$ & $C(43)-C(41)-C(42)$ & $108.9(4)$ \\
\hline$N(5)-S i(2)-C(30)$ & $35.75(16)$ & $N(8)-C(45)-N(7)$ & $116.1(4)$ \\
\hline $\mathrm{Cu}(2)-\mathrm{Si}(2)-\mathrm{C}(30)$ & $151.34(13)$ & $N(8)-C(45)-C(46)$ & $121.2(4)$ \\
\hline $\mathrm{N}(7)-\mathrm{Si}(2)-\mathrm{Cu}(1)$ & $145.57(13)$ & $N(7)-C(45)-C(46)$ & $122.7(4)$ \\
\hline$N(6)-S i(2)-C u(1)$ & $107.26(13)$ & $C(47)-C(46)-C(45)$ & $118.4(4)$ \\
\hline $\mathrm{N}(5)-\mathrm{Si}(2)-\mathrm{Cu}(1)$ & $102.07(12)$ & $C(46)-C(47)-C(48)$ & $120.3(4)$ \\
\hline $\mathrm{Cu}(2)-\mathrm{Si}(2)-\mathrm{Cu}(1)$ & $59.71(4)$ & $C(49)-C(48)-C(47)$ & $117.8(4)$ \\
\hline $\mathrm{C}(30)-\mathrm{Si}(2)-\mathrm{Cu}(1)$ & $104.85(12)$ & $\mathrm{N}(8)-\mathrm{C}(49)-\mathrm{C}(48)$ & $123.2(4)$ \\
\hline $\mathrm{Br}(1)-\mathrm{Cu}(1)-\mathrm{Cu}(2)$ & $139.83(14)$ & $C(51)-C(50)-C(55)$ & $121.1(4)$ \\
\hline $\mathrm{Cl}(1)-\mathrm{Cu}(1)-\mathrm{Cu}(2)$ & $139.7(5)$ & $C(51)-C(50)-N(7)$ & $117.9(4)$ \\
\hline $\mathrm{Br}(1)-\mathrm{Cu}(1)-\mathrm{Cu}(3)$ & $140.24(10)$ & $C(55)-C(50)-N(7)$ & $121.0(4)$ \\
\hline $\mathrm{Cl}(1)-\mathrm{Cu}(1)-\mathrm{Cu}(3)$ & $135.3(4)$ & $C(50)-C(51)-C(52)$ & $118.2(4)$ \\
\hline $\mathrm{Cu}(2)-\mathrm{Cu}(1)-\mathrm{Cu}(3)$ & $62.86(3)$ & $C(50)-C(51)-C(56)$ & $121.6(4)$ \\
\hline $\mathrm{Br}(1)-\mathrm{Cu}(1)-\mathrm{Si}(2)$ & $97.35(13)$ & $C(52)-C(51)-C(56)$ & $120.2(4)$ \\
\hline $\mathrm{Cl}(1)-\mathrm{Cu}(1)-\mathrm{Si}(2)$ & $100.7(4)$ & $C(53)-C(52)-C(51)$ & $121.9(5)$ \\
\hline $\mathrm{Cu}(2)-\mathrm{Cu}(1)-\mathrm{Si}(2)$ & $53.73(4)$ & $C(52)-C(53)-C(54)$ & $118.4(4)$ \\
\hline $\mathrm{Cu}(3)-\mathrm{Cu}(1)-\mathrm{Si}(2)$ & $116.09(4)$ & $C(52)-C(53)-C(57)$ & $120.1(5)$ \\
\hline $\mathrm{Br}(1)-\mathrm{Cu}(1)-\mathrm{Si}(1)$ & $98.46(14)$ & $C(54)-C(53)-C(57)$ & $121.5(5)$ \\
\hline $\mathrm{Cl}(1)-\mathrm{Cu}(1)-\mathrm{Si}(1)$ & $95.7(5)$ & $C(53)-C(54)-C(55)$ & $121.9(5)$ \\
\hline $\mathrm{Cu}(2)-\mathrm{Cu}(1)-\mathrm{Si}(1)$ & $115.89(4)$ & $C(54)-C(55)-C(50)$ & $118.4(5)$ \\
\hline $\mathrm{Cu}(3)-\mathrm{Cu}(1)-\mathrm{Si}(1)$ & $53.72(4)$ & $C(54)-C(55)-C(58)$ & $120.5(4)$ \\
\hline
\end{tabular}




\begin{tabular}{|l|l|l|l|}
\hline $\mathrm{Si}(2)-\mathrm{Cu}(1)-\mathrm{Si}(1)$ & $161.89(4)$ & $\mathrm{C}(50)-\mathrm{C}(55)-\mathrm{C}(58)$ & $121.0(4)$ \\
\hline $\mathrm{N}(8)-\mathrm{Cu}(2)-\mathrm{Si}(2)$ & $85.59(12)$ & $\mathrm{C}(3 \mathrm{~T})-\mathrm{C}(2 \mathrm{~T})-\mathrm{C}(7 \mathrm{~T})$ & $118.5(4)$ \\
\hline $\mathrm{N}(8)-\mathrm{Cu}(2)-\mathrm{Cu}(1)$ & $151.96(12)$ & $\mathrm{C}(3 \mathrm{~T})-\mathrm{C}(2 \mathrm{~T})-\mathrm{C}(1 \mathrm{~T})$ & $119.8(5)$ \\
\hline $\mathrm{Si}(2)-\mathrm{Cu}(2)-\mathrm{Cu}(1)$ & $66.56(4)$ & $\mathrm{C}(7 \mathrm{~T})-\mathrm{C}(2 \mathrm{~T})-\mathrm{C}(1 \mathrm{~T})$ & $121.8(5)$ \\
\hline $\mathrm{N}(8)-\mathrm{Cu}(2)-\mathrm{Br}(2)$ & $99.54(11)$ & $\mathrm{C}(2 \mathrm{~T})-\mathrm{C}(3 \mathrm{~T})-\mathrm{C}(4 \mathrm{~T})$ & $121.1(5)$ \\
\hline $\mathrm{Si}(2)-\mathrm{Cu}(2)-\mathrm{Br}(2)$ & $127.11(4)$ & $\mathrm{C}(3 \mathrm{~T})-\mathrm{C}(4 \mathrm{~T})-\mathrm{C}(5 \mathrm{~T})$ & $120.6(5)$ \\
\hline $\mathrm{Cu}(1)-\mathrm{Cu}(2)-\mathrm{Br}(2)$ & $99.93(3)$ & $\mathrm{C}(6 \mathrm{~T})-\mathrm{C}(5 \mathrm{~T})-\mathrm{C}(4 \mathrm{~T})$ & $120.0(5)$ \\
\hline $\mathrm{N}(8)-\mathrm{Cu}(2)-\mathrm{Br}(3)$ & $107.84(11)$ & $\mathrm{C}(5 \mathrm{~T})-\mathrm{C}(6 \mathrm{~T})-\mathrm{C}(7 \mathrm{~T})$ & $119.7(5)$ \\
\hline & & $\mathrm{C}(6 \mathrm{~T})-\mathrm{C}(7 \mathrm{~T})-\mathrm{C}(2 \mathrm{~T})$ & $120.2(5)$ \\
\hline
\end{tabular}




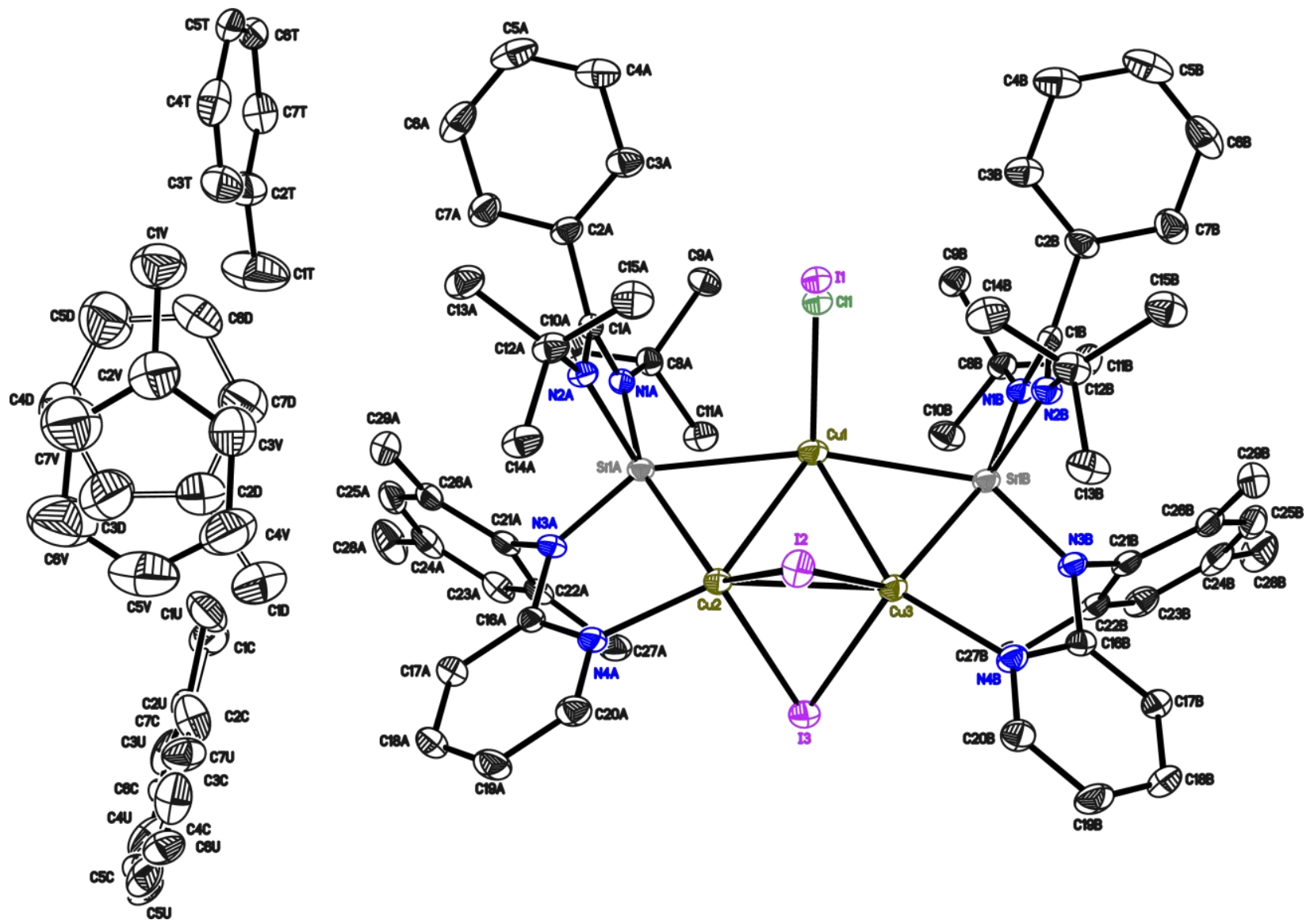

One iodide atom $(11 / \mathrm{Cl} 1)$ was disordered with a chloride atom. The anisotropic displacement parameters are constrained to be identical. The occupancy of the iodide atom refines to $0.0792(13)$.

Partially iodide-chloride-disorder is supposed to stem from a halide-interchange with the chloro benzamidinato silylene $\mathrm{NHSiCl} 1$.

The carbon atoms of two toluene molecules (C1D-C7D/C1V-C7V; C1C-C7C/C1U-C7U) are disordered about two positions. They are refined with distance restraints and restraints for the anisotropic displacement parameters. The occupancies of the minor positions refine to $0.275(7)$ and $0.448(8)$, respectively.

Furthermore, the structure contains certain B-Alerts that were answered in an author response (see Table S2.3). 
Table S2.3: Author response to justify certain B-Alerts.

PLAT232_ALERT_2_B Hirshfeld Test Diff (M-X) Cu1 --Si1A . 17.7 s.u.

PLAT232_ALERT_2_B Hirshfeld Test Diff (M-X) Cu1 --Si1B . 17.0 s.u.

Author Response: This alert vanishes by including Cu1 in the $\mathrm{I} / \mathrm{Cl}$ disorder. However, these two positions are only $0.259 \mathrm{~A}$ apart and this refinement does not lead to an improvement of the model.

Therefore, we remain with the refinement without $\mathrm{Cu}$ disorder.

\section{Bond lengths $[\AA ̊]$ and angles $\left[^{\circ}\right]$ for $3 c$ :}

\begin{tabular}{|c|c|c|c|}
\hline $\mathrm{Cu}(2)-\mathrm{N}(4 \mathrm{~A})$ & $2.014(2)$ & $N(2 A)-S i(1 A)-C(1 A)$ & $35.70(10)$ \\
\hline $\mathrm{Cu}(2)-\mathrm{Si}(1 \mathrm{~A})$ & $2.2819(11)$ & $N(1 A)-S i(1 A)-C(1 A)$ & $35.86(10)$ \\
\hline $\mathrm{Cu}(2)-\mathrm{Cu}(1)$ & $2.4333(9)$ & $\mathrm{Cu}(2)-\mathrm{Si}(1 \mathrm{~A})-\mathrm{C}(1 \mathrm{~A})$ & $152.27(8)$ \\
\hline $\mathrm{Cu}(2)-\mathrm{Cu}(3)$ & $2.5458(5)$ & $\mathrm{N}(3 \mathrm{~A})-\mathrm{Si}(1 \mathrm{~A})-\mathrm{Cu}(1)$ & $145.82(8)$ \\
\hline $\mathrm{Cu}(2)-I(3)$ & $2.6629(6)$ & $\mathrm{N}(2 \mathrm{~A})-\mathrm{Si}(1 \mathrm{~A})-\mathrm{Cu}(1)$ & $106.29(8)$ \\
\hline $\mathrm{Cu}(2)-\mathrm{I}(2)$ & $2.6698(8)$ & $\mathrm{N}(1 \mathrm{~A})-\mathrm{Si}(1 \mathrm{~A})-\mathrm{Cu}(1)$ & $102.59(8)$ \\
\hline $\mathrm{Cu}(3)-\mathrm{N}(4 \mathrm{~B})$ & $2.009(2)$ & $\mathrm{Cu}(2)-\mathrm{Si}(1 \mathrm{~A})-\mathrm{Cu}(1)$ & $59.26(3)$ \\
\hline $\mathrm{Cu}(3)-\mathrm{Si}(1 \mathrm{~B})$ & $2.2838(11)$ & $C(1 A)-S i(1 A)-C u(1)$ & $104.52(7)$ \\
\hline $\mathrm{Cu}(3)-\mathrm{Cu}(1)$ & $2.4227(9)$ & $C(1 A)-N(1 A)-C(8 A)$ & $129.2(2)$ \\
\hline $\mathrm{Cu}(3)-I(2)$ & $2.6668(8)$ & $C(1 A)-N(1 A)-S i(1 A)$ & $90.34(16)$ \\
\hline $\mathrm{Cu}(3)-\mathrm{I}(3)$ & $2.6691(6)$ & $C(8 A)-N(1 A)-S i(1 A)$ & $138.18(17)$ \\
\hline $\mathrm{I}(1)-\mathrm{Cu}(1)$ & $2.550(5)$ & $N(2 A)-C(1 A)-N(1 A)$ & $106.8(2)$ \\
\hline $\mathrm{Cl}(1)-\mathrm{Cu}(1)$ & $2.2184(15)$ & $N(2 A)-C(1 A)-C(2 A)$ & $125.4(2)$ \\
\hline $\mathrm{Cu}(1)-\mathrm{Si}(1 \mathrm{~B})$ & $2.5985(8)$ & $N(1 A)-C(1 A)-C(2 A)$ & $127.8(2)$ \\
\hline $\mathrm{Cu}(1)-\mathrm{Si}(1 \mathrm{~A})$ & $2.6063(8)$ & $N(2 A)-C(1 A)-S i(1 A)$ & $53.54(13)$ \\
\hline $\operatorname{Si}(1 \mathrm{~A})-\mathrm{N}(3 \mathrm{~A})$ & $1.821(2)$ & $N(1 A)-C(1 A)-S i(1 A)$ & $53.81(13)$ \\
\hline $\mathrm{Si}(1 \mathrm{~A})-\mathrm{N}(2 \mathrm{~A})$ & $1.838(2)$ & $C(2 A)-C(1 A)-S i(1 A)$ & $174.2(2)$ \\
\hline $\operatorname{Si}(1 \mathrm{~A})-\mathrm{N}(1 \mathrm{~A})$ & $1.845(2)$ & $\mathrm{N}(3 \mathrm{~B})-\mathrm{Si}(1 \mathrm{~B})-\mathrm{N}(2 \mathrm{~B})$ & $104.49(10)$ \\
\hline $\operatorname{Si}(1 A)-C(1 A)$ & $2.286(3)$ & $N(3 B)-S i(1 B)-N(1 B)$ & $101.18(10)$ \\
\hline$N(1 A)-C(1 A)$ & $1.339(3)$ & $\mathrm{N}(2 \mathrm{~B})-\mathrm{Si}(1 \mathrm{~B})-\mathrm{N}(1 \mathrm{~B})$ & $71.19(10)$ \\
\hline$N(1 A)-C(8 A)$ & $1.490(3)$ & $\mathrm{N}(3 \mathrm{~B})-\mathrm{Si}(1 \mathrm{~B})-\mathrm{Cu}(3)$ & $91.64(8)$ \\
\hline$C(1 A)-N(2 A)$ & $1.334(3)$ & $\mathrm{N}(2 \mathrm{~B})-\mathrm{Si}(1 \mathrm{~B})-\mathrm{Cu}(3)$ & $121.92(8)$ \\
\hline$C(1 A)-C(2 A)$ & $1.492(4)$ & $\mathrm{N}(1 \mathrm{~B})-\mathrm{Si}(1 \mathrm{~B})-\mathrm{Cu}(3)$ & $158.89(8)$ \\
\hline $\mathrm{Si}(1 \mathrm{~B})-\mathrm{N}(3 \mathrm{~B})$ & $1.815(2)$ & $\mathrm{N}(3 \mathrm{~B})-\mathrm{Si}(1 \mathrm{~B})-\mathrm{C}(1 \mathrm{~B})$ & $109.20(10)$ \\
\hline $\mathrm{Si}(1 \mathrm{~B})-\mathrm{N}(2 \mathrm{~B})$ & $1.838(2)$ & $N(2 B)-S i(1 B)-C(1 B)$ & $35.69(10)$ \\
\hline $\mathrm{Si}(1 \mathrm{~B})-\mathrm{N}(1 \mathrm{~B})$ & $1.846(2)$ & $\mathrm{N}(1 \mathrm{~B})-\mathrm{Si}(1 \mathrm{~B})-\mathrm{C}(1 \mathrm{~B})$ & $35.77(10)$ \\
\hline $\mathrm{Si}(1 \mathrm{~B})-\mathrm{C}(1 \mathrm{~B})$ & $2.289(3)$ & $\mathrm{Cu}(3)-\mathrm{Si}(1 \mathrm{~B})-\mathrm{C}(1 \mathrm{~B})$ & $151.80(8)$ \\
\hline$C(1 B)-N(2 B)$ & $1.335(3)$ & $\mathrm{N}(3 \mathrm{~B})-\mathrm{Si}(1 \mathrm{~B})-\mathrm{Cu}(1)$ & $145.11(8)$ \\
\hline$C(1 B)-N(1 B)$ & $1.338(3)$ & $\mathrm{N}(2 \mathrm{~B})-\mathrm{Si}(1 \mathrm{~B})-\mathrm{Cu}(1)$ & $107.16(8)$ \\
\hline$C(1 B)-C(2 B)$ & $1.489(4)$ & $\mathrm{N}(1 \mathrm{~B})-\mathrm{Si}(1 \mathrm{~B})-\mathrm{Cu}(1)$ & $102.57(8)$ \\
\hline$N(1 B)-C(8 B)$ & $1.496(3)$ & $\mathrm{Cu}(3)-\mathrm{Si}(1 \mathrm{~B})-\mathrm{Cu}(1)$ & $59.08(3)$ \\
\hline$C(2 A)-C(7 A)$ & $1.384(4)$ & $\mathrm{C}(1 \mathrm{~B})-\mathrm{Si}(1 \mathrm{~B})-\mathrm{Cu}(1)$ & $105.04(7)$ \\
\hline$C(2 A)-C(3 A)$ & $1.398(4)$ & $\mathrm{N}(2 \mathrm{~B})-\mathrm{C}(1 \mathrm{~B})-\mathrm{N}(1 \mathrm{~B})$ & $106.7(2)$ \\
\hline$N(2 A)-C(12 A)$ & $1.479(3)$ & $N(2 B)-C(1 B)-C(2 B)$ & $124.9(2)$ \\
\hline
\end{tabular}




\begin{tabular}{|c|c|c|c|}
\hline $\mathrm{N}(2 \mathrm{~B})-\mathrm{C}(12 \mathrm{~B})$ & $1.481(3)$ & $N(1 B)-C(1 B)-C(2 B)$ & $128.4(2)$ \\
\hline$C(2 B)-C(7 B)$ & $1.389(4)$ & $N(2 B)-C(1 B)-S i(1 B)$ & $53.44(13)$ \\
\hline$C(2 B)-C(3 B)$ & $1.396(4)$ & $N(1 B)-C(1 B)-S i(1 B)$ & $53.77(13)$ \\
\hline$N(3 A)-C(16 A)$ & $1.390(3)$ & $C(2 B)-C(1 B)-S i(1 B)$ & $173.7(2)$ \\
\hline$N(3 A)-C(21 A)$ & $1.444(3)$ & $C(1 B)-N(1 B)-C(8 B)$ & $128.5(2)$ \\
\hline$C(3 A)-C(4 A)$ & $1.384(4)$ & $C(1 B)-N(1 B)-S i(1 B)$ & $90.46(16)$ \\
\hline$N(4 A)-C(16 A)$ & $1.346(3)$ & $C(8 B)-N(1 B)-S i(1 B)$ & $138.70(18)$ \\
\hline$N(4 A)-C(20 A)$ & $1.347(3)$ & $C(7 A)-C(2 A)-C(3 A)$ & $120.4(3)$ \\
\hline$N(3 B)-C(16 B)$ & $1.387(3)$ & $C(7 A)-C(2 A)-C(1 A)$ & $119.1(3)$ \\
\hline$N(3 B)-C(21 B)$ & $1.441(3)$ & $C(3 A)-C(2 A)-C(1 A)$ & $120.2(2)$ \\
\hline$C(3 B)-C(4 B)$ & $1.383(4)$ & $C(1 A)-N(2 A)-C(12 A)$ & $131.5(2)$ \\
\hline$C(5 A)-C(4 A)$ & $1.373(5)$ & $C(1 A)-N(2 A)-S i(1 A)$ & $90.77(16)$ \\
\hline$C(5 A)-C(6 A)$ & $1.386(5)$ & $C(12 A)-N(2 A)-S i(1 A)$ & $136.95(18)$ \\
\hline$N(4 B)-C(16 B)$ & $1.341(3)$ & $C(1 B)-N(2 B)-C(12 B)$ & $131.5(2)$ \\
\hline$N(4 B)-C(20 B)$ & $1.350(4)$ & $C(1 B)-N(2 B)-S i(1 B)$ & $90.87(17)$ \\
\hline$C(4 B)-C(5 B)$ & $1.378(5)$ & $C(12 B)-N(2 B)-S i(1 B)$ & $136.91(18)$ \\
\hline$C(6 A)-C(7 A)$ & $1.394(4)$ & $C(7 B)-C(2 B)-C(3 B)$ & $120.4(3)$ \\
\hline$C(5 B)-C(6 B)$ & $1.378(5)$ & $C(7 B)-C(2 B)-C(1 B)$ & $119.3(3)$ \\
\hline$C(6 B)-C(7 B)$ & $1.392(4)$ & $C(3 B)-C(2 B)-C(1 B)$ & $120.1(3)$ \\
\hline$C(8 A)-C(11 A)$ & $1.523(4)$ & $C(16 A)-N(3 A)-C(21 A)$ & $115.6(2)$ \\
\hline$C(8 A)-C(10 A)$ & $1.528(4)$ & $C(16 A)-N(3 A)-S i(1 A)$ & $118.80(18)$ \\
\hline$C(8 A)-C(9 A)$ & $1.533(4)$ & $C(21 A)-N(3 A)-S i(1 A)$ & $125.35(17)$ \\
\hline$C(8 B)-C(9 B)$ & $1.519(4)$ & $C(4 A)-C(3 A)-C(2 A)$ & $119.2(3)$ \\
\hline$C(8 B)-C(10 B)$ & $1.526(4)$ & $C(16 A)-N(4 A)-C(20 A)$ & $119.2(2)$ \\
\hline$C(8 B)-C(11 B)$ & $1.527(4)$ & $C(16 A)-N(4 A)-C u(2)$ & $117.14(17)$ \\
\hline$C(12 A)-C(14 A)$ & $1.528(4)$ & $C(20 A)-N(4 A)-C u(2)$ & $122.55(19)$ \\
\hline$C(12 A)-C(13 A)$ & $1.530(4)$ & $C(16 B)-N(3 B)-C(21 B)$ & $115.6(2)$ \\
\hline$C(12 A)-C(15 A)$ & $1.535(4)$ & $C(16 B)-N(3 B)-S i(1 B)$ & $119.26(18)$ \\
\hline$C(12 B)-C(14 B)$ & $1.529(4)$ & $C(21 B)-N(3 B)-S i(1 B)$ & $124.69(17)$ \\
\hline$C(12 B)-C(13 B)$ & $1.529(4)$ & $C(4 B)-C(3 B)-C(2 B)$ & $119.5(3)$ \\
\hline$C(12 B)-C(15 B)$ & $1.532(4)$ & $C(4 A)-C(5 A)-C(6 A)$ & $120.6(3)$ \\
\hline$C(16 A)-C(17 A)$ & $1.405(4)$ & $\mathrm{C}(16 \mathrm{~B})-\mathrm{N}(4 \mathrm{~B})-\mathrm{C}(20 \mathrm{~B})$ & $119.4(2)$ \\
\hline$C(17 A)-C(18 A)$ & $1.376(4)$ & $C(16 B)-N(4 B)-C u(3)$ & $117.03(17)$ \\
\hline$C(16 B)-C(17 B)$ & $1.403(4)$ & $C(20 B)-N(4 B)-C u(3)$ & $122.11(19)$ \\
\hline$C(18 A)-C(19 A)$ & $1.383(4)$ & $C(5 B)-C(4 B)-C(3 B)$ & $119.9(3)$ \\
\hline$C(17 B)-C(18 B)$ & $1.373(4)$ & $C(5 A)-C(6 A)-C(7 A)$ & $119.7(3)$ \\
\hline$C(19 A)-C(20 A)$ & $1.371(4)$ & $C(6 B)-C(5 B)-C(4 B)$ & $120.9(3)$ \\
\hline$C(18 B)-C(19 B)$ & $1.392(4)$ & $C(2 A)-C(7 A)-C(6 A)$ & $119.5(3)$ \\
\hline$C(19 B)-C(20 B)$ & $1.368(4)$ & $C(5 B)-C(6 B)-C(7 B)$ & $119.9(3)$ \\
\hline$C(21 A)-C(26 A)$ & $1.400(4)$ & $N(1 A)-C(8 A)-C(11 A)$ & $106.2(2)$ \\
\hline$C(21 A)-C(22 A)$ & $1.403(4)$ & $N(1 A)-C(8 A)-C(10 A)$ & $110.5(2)$ \\
\hline$C(22 A)-C(23 A)$ & $1.392(4)$ & $C(11 A)-C(8 A)-C(10 A)$ & $109.6(2)$ \\
\hline$C(22 A)-C(27 A)$ & $1.500(4)$ & $N(1 A)-C(8 A)-C(9 A)$ & $111.7(2)$ \\
\hline$C(21 B)-C(26 B)$ & $1.399(4)$ & $C(11 A)-C(8 A)-C(9 A)$ & $109.0(2)$ \\
\hline
\end{tabular}




\begin{tabular}{|c|c|c|c|}
\hline$C(21 B)-C(22 B)$ & $1.404(4)$ & $C(10 A)-C(8 A)-C(9 A)$ & $109.7(2)$ \\
\hline$C(23 A)-C(24 A)$ & $1.382(4)$ & $C(2 B)-C(7 B)-C(6 B)$ & $119.2(3)$ \\
\hline$C(22 B)-C(23 B)$ & $1.393(4)$ & $N(1 B)-C(8 B)-C(9 B)$ & $112.5(2)$ \\
\hline$C(22 B)-C(27 B)$ & $1.505(4)$ & $N(1 B)-C(8 B)-C(10 B)$ & $105.9(2)$ \\
\hline$C(24 A)-C(25 A)$ & $1.399(5)$ & $C(9 B)-C(8 B)-C(10 B)$ & $108.9(2)$ \\
\hline$C(24 A)-C(28 A)$ & $1.496(4)$ & $\mathrm{N}(1 \mathrm{~B})-\mathrm{C}(8 \mathrm{~B})-\mathrm{C}(11 \mathrm{~B})$ & $109.6(2)$ \\
\hline$C(23 B)-C(24 B)$ & $1.381(4)$ & $C(9 B)-C(8 B)-C(11 B)$ & $110.2(2)$ \\
\hline$C(25 A)-C(26 A)$ & $1.392(4)$ & $C(10 B)-C(8 B)-C(11 B)$ & $109.7(2)$ \\
\hline$C(24 B)-C(25 B)$ & $1.386(5)$ & $N(2 A)-C(12 A)-C(14 A)$ & $106.1(2)$ \\
\hline$C(24 B)-C(28 B)$ & $1.505(4)$ & $N(2 A)-C(12 A)-C(13 A)$ & $111.9(2)$ \\
\hline$C(26 A)-C(29 A)$ & $1.503(4)$ & $C(14 A)-C(12 A)-C(13 A)$ & $109.1(2)$ \\
\hline$C(25 B)-C(26 B)$ & $1.401(4)$ & $N(2 A)-C(12 A)-C(15 A)$ & $110.2(2)$ \\
\hline$C(26 B)-C(29 B)$ & $1.500(4)$ & $C(14 A)-C(12 A)-C(15 A)$ & $109.2(2)$ \\
\hline$C(1 T)-C(2 T)$ & $1.506(5)$ & $C(13 A)-C(12 A)-C(15 A)$ & $110.2(2)$ \\
\hline$C(2 T)-C(3 T)$ & $1.396(5)$ & $N(2 B)-C(12 B)-C(14 B)$ & $110.3(2)$ \\
\hline$C(2 T)-C(7 T)$ & $1.398(5)$ & $N(2 B)-C(12 B)-C(13 B)$ & $106.0(2)$ \\
\hline$C(3 T)-C(4 T)$ & $1.363(5)$ & $C(14 B)-C(12 B)-C(13 B)$ & $109.3(2)$ \\
\hline$C(4 T)-C(5 T)$ & $1.385(5)$ & $N(2 B)-C(12 B)-C(15 B)$ & $111.7(2)$ \\
\hline$C(5 T)-C(6 T)$ & $1.380(5)$ & $C(14 B)-C(12 B)-C(15 B)$ & $110.5(2)$ \\
\hline$C(6 T)-C(7 T)$ & $1.381(4)$ & $C(13 B)-C(12 B)-C(15 B)$ & $108.8(2)$ \\
\hline$C(1 V)-C(2 V)$ & $1.516(7)$ & $N(4 A)-C(16 A)-N(3 A)$ & $116.2(2)$ \\
\hline$C(2 V)-C(7 V)$ & $1.390(9)$ & $N(4 A)-C(16 A)-C(17 A)$ & $120.8(2)$ \\
\hline$C(2 V)-C(3 V)$ & $1.390(8)$ & $N(3 A)-C(16 A)-C(17 A)$ & $123.0(2)$ \\
\hline$C(3 V)-C(4 V)$ & $1.385(8)$ & $C(18 A)-C(17 A)-C(16 A)$ & $118.7(3)$ \\
\hline$C(4 V)-C(5 V)$ & $1.354(8)$ & $\mathrm{N}(4 \mathrm{~B})-\mathrm{C}(16 \mathrm{~B})-\mathrm{N}(3 \mathrm{~B})$ & $116.4(2)$ \\
\hline$C(5 V)-C(6 V)$ & $1.397(9)$ & $N(4 B)-C(16 B)-C(17 B)$ & $120.6(2)$ \\
\hline$C(6 V)-C(7 V)$ & $1.358(9)$ & $\mathrm{N}(3 \mathrm{~B})-\mathrm{C}(16 \mathrm{~B})-\mathrm{C}(17 \mathrm{~B})$ & $123.0(2)$ \\
\hline$C(1 D)-C(2 D)$ & $1.511(13)$ & $C(17 A)-C(18 A)-C(19 A)$ & $120.3(3)$ \\
\hline$C(2 D)-C(7 D)$ & $1.385(12)$ & $C(18 B)-C(17 B)-C(16 B)$ & 119.3(3) \\
\hline$C(2 D)-C(3 D)$ & $1.391(12)$ & $C(20 A)-C(19 A)-C(18 A)$ & 118.1(3) \\
\hline$C(3 D)-C(4 D)$ & $1.376(13)$ & $C(17 B)-C(18 B)-C(19 B)$ & $119.7(3)$ \\
\hline$C(4 D)-C(5 D)$ & $1.394(13)$ & $N(4 A)-C(20 A)-C(19 A)$ & $122.8(3)$ \\
\hline$C(5 D)-C(6 D)$ & $1.371(12)$ & $C(20 B)-C(19 B)-C(18 B)$ & $118.2(3)$ \\
\hline$C(6 D)-C(7 D)$ & $1.385(13)$ & $C(26 A)-C(21 A)-C(22 A)$ & $120.8(3)$ \\
\hline$C(1 U)-C(2 U)$ & $1.489(8)$ & $C(26 A)-C(21 A)-N(3 A)$ & $121.2(2)$ \\
\hline$C(2 U)-C(3 U)$ & $1.364(9)$ & $C(22 A)-C(21 A)-N(3 A)$ & $117.9(2)$ \\
\hline$C(2 U)-C(7 U)$ & $1.377(9)$ & $N(4 B)-C(20 B)-C(19 B)$ & $122.7(3)$ \\
\hline$C(3 U)-C(4 U)$ & $1.351(10)$ & $C(23 A)-C(22 A)-C(21 A)$ & $118.4(3)$ \\
\hline$C(4 U)-C(5 U)$ & $1.378(9)$ & $C(23 A)-C(22 A)-C(27 A)$ & $119.7(3)$ \\
\hline$C(5 U)-C(6 U)$ & $1.377(9)$ & $C(21 A)-C(22 A)-C(27 A)$ & $121.9(2)$ \\
\hline$C(6 U)-C(7 U)$ & $1.374(9)$ & $C(26 B)-C(21 B)-C(22 B)$ & $120.8(3)$ \\
\hline$C(1 C)-C(2 C)$ & $1.494(9)$ & $C(26 B)-C(21 B)-N(3 B)$ & $121.3(3)$ \\
\hline$C(2 C)-C(7 C)$ & $1.377(9)$ & $C(22 B)-C(21 B)-N(3 B)$ & $117.9(2)$ \\
\hline$C(2 C)-C(3 C)$ & $1.381(9)$ & $C(24 A)-C(23 A)-C(22 A)$ & $122.2(3)$ \\
\hline
\end{tabular}




\begin{tabular}{|c|c|c|c|}
\hline$C(3 C)-C(4 C)$ & $1.373(10)$ & $C(23 B)-C(22 B)-C(21 B)$ & $118.4(3)$ \\
\hline$C(4 C)-C(5 C)$ & $1.386(11)$ & $C(23 B)-C(22 B)-C(27 B)$ & $120.6(3)$ \\
\hline$C(5 C)-C(6 C)$ & $1.365(10)$ & $C(21 B)-C(22 B)-C(27 B)$ & $121.0(2)$ \\
\hline \multirow[t]{2}{*}{$C(6 C)-C(7 C)$} & $1.373(9)$ & $C(23 A)-C(24 A)-C(25 A)$ & $118.2(3)$ \\
\hline & & $C(23 A)-C(24 A)-C(28 A)$ & $120.1(3)$ \\
\hline$N(4 A)-C u(2)-S i(1 A)$ & $84.83(7)$ & $C(25 A)-C(24 A)-C(28 A)$ & $121.6(3)$ \\
\hline $\mathrm{N}(4 \mathrm{~A})-\mathrm{Cu}(2)-\mathrm{Cu}(1)$ & $151.04(6)$ & $C(24 B)-C(23 B)-C(22 B)$ & $122.1(3)$ \\
\hline $\mathrm{Si}(1 \mathrm{~A})-\mathrm{Cu}(2)-\mathrm{Cu}(1)$ & $67.02(3)$ & $C(26 A)-C(25 A)-C(24 A)$ & $121.6(3)$ \\
\hline$N(4 A)-C u(2)-C u(3)$ & $150.65(6)$ & $C(23 B)-C(24 B)-C(25 B)$ & $118.2(3)$ \\
\hline $\mathrm{Si}(1 \mathrm{~A})-\mathrm{Cu}(2)-\mathrm{Cu}(3)$ & $124.33(3)$ & $C(23 B)-C(24 B)-C(28 B)$ & $121.0(3)$ \\
\hline $\mathrm{Cu}(1)-\mathrm{Cu}(2)-\mathrm{Cu}(3)$ & $58.18(3)$ & $C(25 B)-C(24 B)-C(28 B)$ & $120.9(3)$ \\
\hline $\mathrm{N}(4 \mathrm{~A})-\mathrm{Cu}(2)-\mathrm{I}(3)$ & $100.57(6)$ & $C(25 A)-C(26 A)-C(21 A)$ & $118.6(3)$ \\
\hline $\operatorname{Si}(1 \mathrm{~A})-\mathrm{Cu}(2)-\mathrm{I}(3)$ & $125.22(3)$ & $C(25 A)-C(26 A)-C(29 A)$ & $120.5(3)$ \\
\hline $\mathrm{Cu}(1)-\mathrm{Cu}(2)-\mathrm{I}(3)$ & $101.24(2)$ & $C(21 A)-C(26 A)-C(29 A)$ & $120.9(3)$ \\
\hline $\mathrm{Cu}(3)-\mathrm{Cu}(2)-\mathrm{I}(3)$ & $61.603(19)$ & $C(24 B)-C(25 B)-C(26 B)$ & $122.2(3)$ \\
\hline $\mathrm{N}(4 \mathrm{~A})-\mathrm{Cu}(2)-\mathrm{I}(2)$ & $106.58(7)$ & $C(21 B)-C(26 B)-C(25 B)$ & $118.0(3)$ \\
\hline $\mathrm{Si}(1 \mathrm{~A})-\mathrm{Cu}(2)-\mathrm{I}(2)$ & $125.19(3)$ & $C(21 B)-C(26 B)-C(29 B)$ & $121.0(3)$ \\
\hline $\mathrm{Cu}(1)-\mathrm{Cu}(2)-\mathrm{I}(2)$ & $85.58(2)$ & $C(25 B)-C(26 B)-C(29 B)$ & $120.9(3)$ \\
\hline $\mathrm{Cu}(3)-\mathrm{Cu}(2)-\mathrm{I}(2)$ & $61.45(2)$ & $C(5 A)-C(4 A)-C(3 A)$ & $120.5(3)$ \\
\hline $\mathrm{I}(3)-\mathrm{Cu}(2)-\mathrm{I}(2)$ & $105.53(2)$ & $C(3 T)-C(2 T)-C(7 T)$ & $118.4(3)$ \\
\hline $\mathrm{N}(4 \mathrm{~B})-\mathrm{Cu}(3)-\mathrm{Si}(1 \mathrm{~B})$ & $84.92(7)$ & $C(3 T)-C(2 T)-C(1 T)$ & $123.0(3)$ \\
\hline $\mathrm{N}(4 \mathrm{~B})-\mathrm{Cu}(3)-\mathrm{Cu}(1)$ & $151.45(7)$ & $C(7 T)-C(2 T)-C(1 T)$ & $118.6(4)$ \\
\hline $\mathrm{Si}(1 \mathrm{~B})-\mathrm{Cu}(3)-\mathrm{Cu}(1)$ & $66.95(3)$ & $C(4 T)-C(3 T)-C(2 T)$ & $120.7(3)$ \\
\hline $\mathrm{N}(4 \mathrm{~B})-\mathrm{Cu}(3)-\mathrm{Cu}(2)$ & $149.96(7)$ & $C(3 T)-C(4 T)-C(5 T)$ & $120.9(3)$ \\
\hline $\mathrm{Si}(1 \mathrm{~B})-\mathrm{Cu}(3)-\mathrm{Cu}(2)$ & $124.80(3)$ & $C(6 T)-C(5 T)-C(4 T)$ & $119.2(3)$ \\
\hline $\mathrm{Cu}(1)-\mathrm{Cu}(3)-\mathrm{Cu}(2)$ & $58.59(3)$ & $C(5 T)-C(6 T)-C(7 T)$ & $120.6(3)$ \\
\hline $\mathrm{N}(4 \mathrm{~B})-\mathrm{Cu}(3)-\mathrm{I}(2)$ & $107.54(7)$ & $C(6 T)-C(7 T)-C(2 T)$ & $120.2(3)$ \\
\hline $\mathrm{Si}(1 \mathrm{~B})-\mathrm{Cu}(3)-\mathrm{I}(2)$ & $124.48(3)$ & $C(7 V)-C(2 V)-C(3 V)$ & $116.7(6)$ \\
\hline $\mathrm{Cu}(1)-\mathrm{Cu}(3)-\mathrm{I}(2)$ & $85.86(2)$ & $C(7 V)-C(2 V)-C(1 V)$ & $121.4(6)$ \\
\hline $\mathrm{Cu}(2)-\mathrm{Cu}(3)-\mathrm{I}(2)$ & $61.57(2)$ & $C(3 V)-C(2 V)-C(1 V)$ & $121.9(6)$ \\
\hline $\mathrm{N}(4 \mathrm{~B})-\mathrm{Cu}(3)-1(3)$ & $99.16(7)$ & $C(4 V)-C(3 V)-C(2 V)$ & $121.6(7)$ \\
\hline $\mathrm{Si}(1 \mathrm{~B})-\mathrm{Cu}(3)-\mathrm{I}(3)$ & $126.20(3)$ & $C(5 V)-C(4 V)-C(3 V)$ & $118.6(7)$ \\
\hline $\mathrm{Cu}(1)-\mathrm{Cu}(3)-\mathrm{I}(3)$ & $101.34(2)$ & $C(4 V)-C(5 V)-C(6 V)$ & $122.5(6)$ \\
\hline $\mathrm{Cu}(2)-\mathrm{Cu}(3)-\mathrm{I}(3)$ & $61.36(2)$ & $C(7 V)-C(6 V)-C(5 V)$ & $117.1(8)$ \\
\hline $\mathrm{I}(2)-\mathrm{Cu}(3)-\mathrm{I}(3)$ & $105.44(2)$ & $C(6 V)-C(7 V)-C(2 V)$ & $123.4(8)$ \\
\hline $\mathrm{Cl}(1)-\mathrm{Cu}(1)-\mathrm{Cu}(3)$ & $141.62(4)$ & $C(7 D)-C(2 D)-C(3 D)$ & $115.2(12)$ \\
\hline $\mathrm{Cl}(1)-\mathrm{Cu}(1)-\mathrm{Cu}(2)$ & $141.22(4)$ & $C(7 D)-C(2 D)-C(1 D)$ & $119.6(12)$ \\
\hline $\mathrm{Cu}(3)-\mathrm{Cu}(1)-\mathrm{Cu}(2)$ & $63.24(2)$ & $C(3 D)-C(2 D)-C(1 D)$ & $125.1(13)$ \\
\hline $\mathrm{Cu}(3)-\mathrm{Cu}(1)-\mathrm{I}(1)$ & $137.44(12)$ & $C(4 D)-C(3 D)-C(2 D)$ & 119.2(13) \\
\hline $\mathrm{Cu}(2)-\mathrm{Cu}(1)-\mathrm{I}(1)$ & $136.76(12)$ & $C(3 D)-C(4 D)-C(5 D)$ & $124.2(15)$ \\
\hline $\mathrm{Cl}(1)-\mathrm{Cu}(1)-\mathrm{Si}(1 \mathrm{~B})$ & $97.59(5)$ & $C(6 D)-C(5 D)-C(4 D)$ & 117.1(13) \\
\hline $\mathrm{Cu}(3)-\mathrm{Cu}(1)-\mathrm{Si}(1 \mathrm{~B})$ & $53.97(3)$ & $C(5 D)-C(6 D)-C(7 D)$ & $117.8(13)$ \\
\hline $\mathrm{Cu}(2)-\mathrm{Cu}(1)-\mathrm{Si}(1 \mathrm{~B})$ & $116.59(3)$ & $C(6 D)-C(7 D)-C(2 D)$ & $126.0(14)$ \\
\hline $\mathrm{I}(1)-\mathrm{Cu}(1)-\mathrm{Si}(1 \mathrm{~B})$ & $98.38(12)$ & $C(3 U)-C(2 U)-C(7 U)$ & $119.2(7)$ \\
\hline
\end{tabular}




\begin{tabular}{|l|l|l|l|}
\hline $\mathrm{Cl}(1)-\mathrm{Cu}(1)-\mathrm{Si}(1 \mathrm{~A})$ & $97.69(5)$ & $\mathrm{C}(3 \mathrm{U})-\mathrm{C}(2 \mathrm{U})-\mathrm{C}(1 \mathrm{U})$ & $120.2(10)$ \\
\hline $\mathrm{Cu}(3)-\mathrm{Cu}(1)-\mathrm{Si}(1 \mathrm{~A})$ & $116.21(3)$ & $\mathrm{C}(7 \mathrm{U})-\mathrm{C}(2 \mathrm{U})-\mathrm{C}(1 \mathrm{U})$ & $120.5(10)$ \\
\hline $\mathrm{Cu}(2)-\mathrm{Cu}(1)-\mathrm{Si}(1 \mathrm{~A})$ & $53.71(3)$ & $\mathrm{C}(4 \mathrm{U})-\mathrm{C}(3 \mathrm{U})-\mathrm{C}(2 \mathrm{U})$ & $120.0(7)$ \\
\hline $\mathrm{I}(1)-\mathrm{Cu}(1)-\mathrm{Si}(1 \mathrm{~A})$ & $98.14(12)$ & $\mathrm{C}(3 \mathrm{U})-\mathrm{C}(4 \mathrm{U})-\mathrm{C}(5 \mathrm{U})$ & $121.7(8)$ \\
\hline $\mathrm{Si}(1 \mathrm{~B})-\mathrm{Cu}(1)-\mathrm{Si}(1 \mathrm{~A})$ & $161.36(3)$ & $\mathrm{C}(6 \mathrm{U})-\mathrm{C}(5 \mathrm{U})-\mathrm{C}(4 \mathrm{U})$ & $118.9(8)$ \\
\hline $\mathrm{Cu}(3)-\mathrm{I}(2)-\mathrm{Cu}(2)$ & $56.986(16)$ & $\mathrm{C}(7 \mathrm{U})-\mathrm{C}(6 \mathrm{U})-\mathrm{C}(5 \mathrm{U})$ & $119.1(7)$ \\
\hline $\mathrm{Cu}(2)-\mathrm{I}(3)-\mathrm{Cu}(3)$ & $57.039(12)$ & $\mathrm{C}(6 \mathrm{U})-\mathrm{C}(7 \mathrm{U})-\mathrm{C}(2 \mathrm{U})$ & $121.1(7)$ \\
\hline $\mathrm{N}(3 \mathrm{~A})-\mathrm{Si}(1 \mathrm{~A})-\mathrm{N}(2 \mathrm{~A})$ & $104.53(10)$ & $\mathrm{C}(7 \mathrm{C})-\mathrm{C}(2 \mathrm{C})-\mathrm{C}(3 \mathrm{C})$ & $118.0(8)$ \\
\hline $\mathrm{N}(3 \mathrm{~A})-\mathrm{Si}(1 \mathrm{~A})-\mathrm{N}(1 \mathrm{~A})$ & $100.96(10)$ & $\mathrm{C}(7 \mathrm{C})-\mathrm{C}(2 \mathrm{C})-\mathrm{C}(1 \mathrm{C})$ & $121.3(10)$ \\
\hline $\mathrm{N}(2 \mathrm{~A})-\mathrm{Si}(1 \mathrm{~A})-\mathrm{N}(1 \mathrm{~A})$ & $71.28(10)$ & $\mathrm{C}(3 \mathrm{C})-\mathrm{C}(2 \mathrm{C})-\mathrm{C}(1 \mathrm{C})$ & $120.6(11)$ \\
\hline $\mathrm{N}(3 \mathrm{~A})-\mathrm{Si}(1 \mathrm{~A})-\mathrm{Cu}(2)$ & $91.60(7)$ & $\mathrm{C}(4 \mathrm{C})-\mathrm{C}(3 \mathrm{C})-\mathrm{C}(2 \mathrm{C})$ & $119.4(9)$ \\
\hline $\mathrm{N}(2 \mathrm{~A})-\mathrm{Si}(1 \mathrm{~A})-\mathrm{Cu}(2)$ & $122.44(8)$ & $\mathrm{C}(3 \mathrm{C})-\mathrm{C}(4 \mathrm{C})-\mathrm{C}(5 \mathrm{C})$ & $122.0(9)$ \\
\hline $\mathrm{N}(1 \mathrm{~A})-\mathrm{Si}(1 \mathrm{~A})-\mathrm{Cu}(2)$ & $158.57(8)$ & $\mathrm{C}(6 \mathrm{C})-\mathrm{C}(5 \mathrm{C})-\mathrm{C}(4 \mathrm{C})$ & $118.4(8)$ \\
\hline $\mathrm{N}(3 \mathrm{~A})-\mathrm{Si}(1 \mathrm{~A})-\mathrm{C}(1 \mathrm{~A})$ & $109.09(10)$ & $\mathrm{C}(5 \mathrm{C})-\mathrm{C}(6 \mathrm{C})-\mathrm{C}(7 \mathrm{C})$ & $119.6(9)$ \\
\hline & & $\mathrm{C}(6 \mathrm{C})-\mathrm{C}(7 \mathrm{C})-\mathrm{C}(2 \mathrm{C})$ & $122.5(8)$ \\
\hline
\end{tabular}




\section{S3. NMR Spectroscopic Analysis}

All spectra were recorded either on a Bruker Avance III $400 \mathrm{MHz}$ spectrometer with a BBFO probe, z-gradient (maximum gradient strength: $57 \mathrm{G} / \mathrm{cm}$ ) or on a Bruker Ascend $400 \mathrm{MHz}$ spectrometer with a BBI probe, z-gradient (maximum gradient strength: $51 \mathrm{G} / \mathrm{cm}$ ). If not indicated otherwise experiments were done at ambient temperature and without spinning. Relevant parameters are given in Table S3.1. Spectra processing was carried out using Topspin 3.5. The diffusion coefficients were determined with the T1/T2 relaxation module.

Table S3.1: Parameters of the DOSY-NMR-Experiments.

\begin{tabular}{cc}
\hline PulseProgram & dstebpgp3s \\
\hline$\Delta$ & $1.1-3.4 \mathrm{~ms}$ \\
$\delta$ & $0.1 \mathrm{~s}$ \\
\hline
\end{tabular}

Data evaluation was performed using the $M W$ Estimation Software Version 1.3 in consideration of the van-der-Waals corrected ECC-DOSY. ${ }^{[6]}$

Table S3.2: Linear fit parameters $(\log (K)$ and $\alpha)$ as well as $\log \left(D_{\text {ref,fix }}\right)$ for THF- $d_{8 .}{ }^{[7]}$

\begin{tabular}{cc}
\hline & THF- $d_{8}$ \\
\hline $\log \left(D_{\text {ref,fix }}\right)$ & -8.7749 \\
& $($ TMB $)$ \\
$\log (K)$ & -7.60 \\
$-\alpha$ & -0.553 \\
\hline
\end{tabular}

Table S3.3: Fit parameters of the van-der-Waals corrected ECC-DOSY for THF- $d_{8}$ with the general formula: ${ }^{[8]}$

\begin{tabular}{cc}
$\left.X_{\text {cor }}=\frac{a}{\left(1+\exp \left(-k\left(M D_{\mathrm{W}}-x c\right)\right)\right.}\right)$ \\
\hline Parameter \\
\hline$a$ & 2.255 \\
$k$ & $2.97 \cdot 10^{-}$ \\
& 30 \\
$x c$ & $5.12 \cdot 10^{29}$ \\
cor. $\mathrm{R}^{2}$ & 0.93 \\
\hline
\end{tabular}




\section{DOSY measurements}

$\underline{\text { ECC-MW Estimation of (NHSiMes) }{ }_{2}(\mathrm{CuBr})_{3}}$

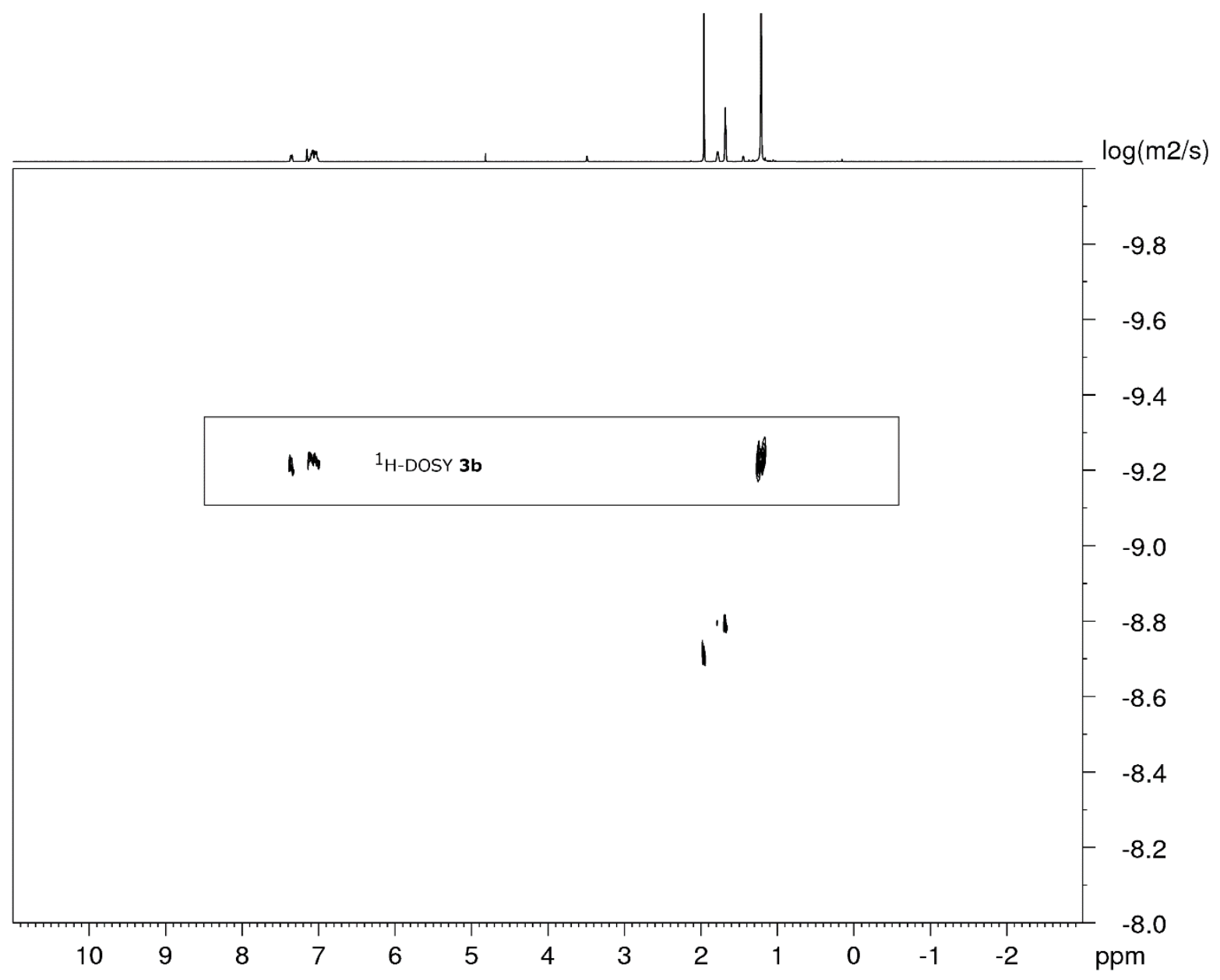

Figure S3.1: ${ }^{1} \mathrm{H}$-DOSY of the complex $\mathbf{3 b}$ in a solution of Benzene- $d_{6}$ and THF- $d_{8}$ with Adamantane as internal reference. 
Table S3.4: ${ }^{1} \mathrm{H}$-DOSY-ECC-MW estimation regarding the ECC-MD $D_{W}$ correction of $\mathbf{3 b}$ in a solution of Benzene- $d_{6}$ and THF- $d_{8}$. Adamantane $\left(\log D_{\text {ref,fix }}=-8,8025\right)$ was used as internal reference. Hypothetical aggregates are marked from $A$ to $D$. However the data indicate a decomposition of the from $X$-ray experiments determined tetrametallic species $D$ in solution into a bimetallic species $(A-C)$. Free coordination sides might be occupied by solvent molecules as the slightly elevated molecular weight compared to the solvent free aggregate A indicates.

\begin{tabular}{|c|c|c|c|c|c|c|}
\hline${ }^{1} \mathrm{H}$-DOSY & & & & & & \\
\hline & & Aggregate & $M W_{\text {det }}[\mathrm{g} / \mathrm{mol}]$ & $M W_{\text {dif }}[\%]$ & $M D_{\mathrm{w}}\left[\mathrm{gmol}^{-1} \mathrm{~m}^{-3}\right]$ & $X_{\text {cor }}$ \\
\hline$D_{\mathrm{x}}\left[\mathrm{m}^{2} / \mathrm{s}\right]$ & $5.92 \mathrm{E}-10$ & A & 1023 & -4 & $6.21 \mathrm{E}+29$ & 1.3556 \\
\hline $\log \left(D_{x}\right)$ & -9.2277 & B & 998 & -5 & $6.02 \mathrm{E}+29$ & 1.3222 \\
\hline $\log \left(D_{x, \text { norm }}\right)$ & -9.2261 & C & 997 & 4 & $5.86 \mathrm{E}+29$ & 1.2852 \\
\hline $\begin{array}{l}D_{\text {ref }} \\
(A D A M)\left[\mathrm{m}^{2} / \mathrm{s}\right]\end{array}$ & $1.57 \mathrm{E}-09$ & D & 1023 & 71 & $6.21 E+29$ & 1.3556 \\
\hline $\log \left(D_{\text {ref }}\right)(A D A M)$ & -8.8041 & & & & & \\
\hline
\end{tabular}

A

$\left(M W_{\text {calc }}=877 \mathrm{gmol}^{-1}\right)$

B

$\left(M W_{\text {calc }}=949 \mathrm{gmol}^{-1}\right)$

C

$\left(M W_{\text {calc }}=1021 \mathrm{gmol}^{-1}\right)$
Structure

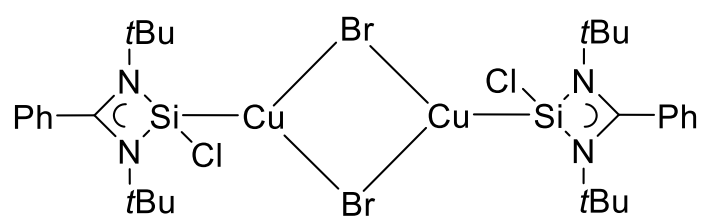<smiles></smiles>

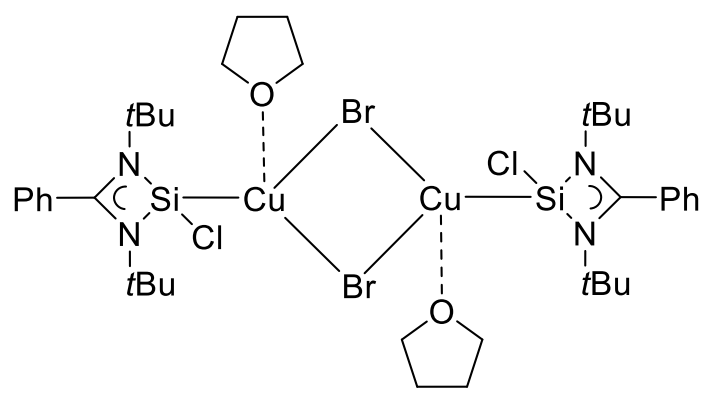




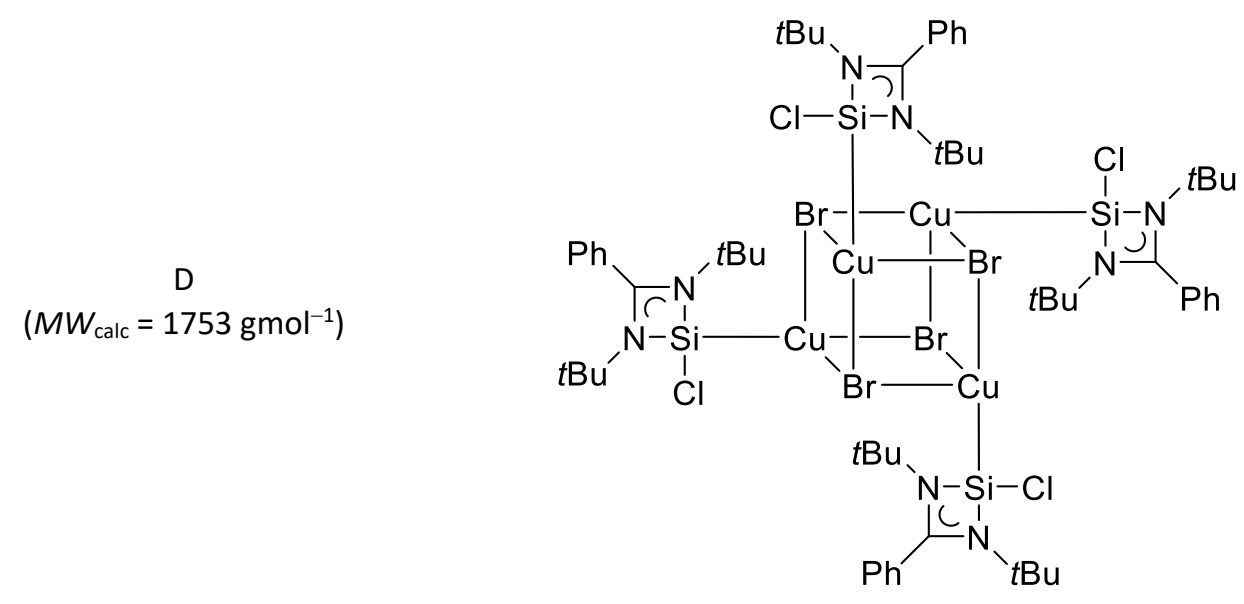




\section{CuAAC Reactions}

General Procedure for Catalysis A NMR Young tube was charged with benzyl azide (1.00 eq.), the appropriate alkyne (1.00 eq), adamantane as an internal standard and deuterated dichloromethane. After an initial measurement of the neat reactants, the complex $\mathbf{2} \mathbf{b} \mathbf{b} \mathbf{3} \mathbf{b}$ was added. The progress of the reaction was monitored via ${ }^{1} \mathrm{H}$ NMR spectroscopic measurements in 10 min steps for at least $6 \mathrm{~h}$.

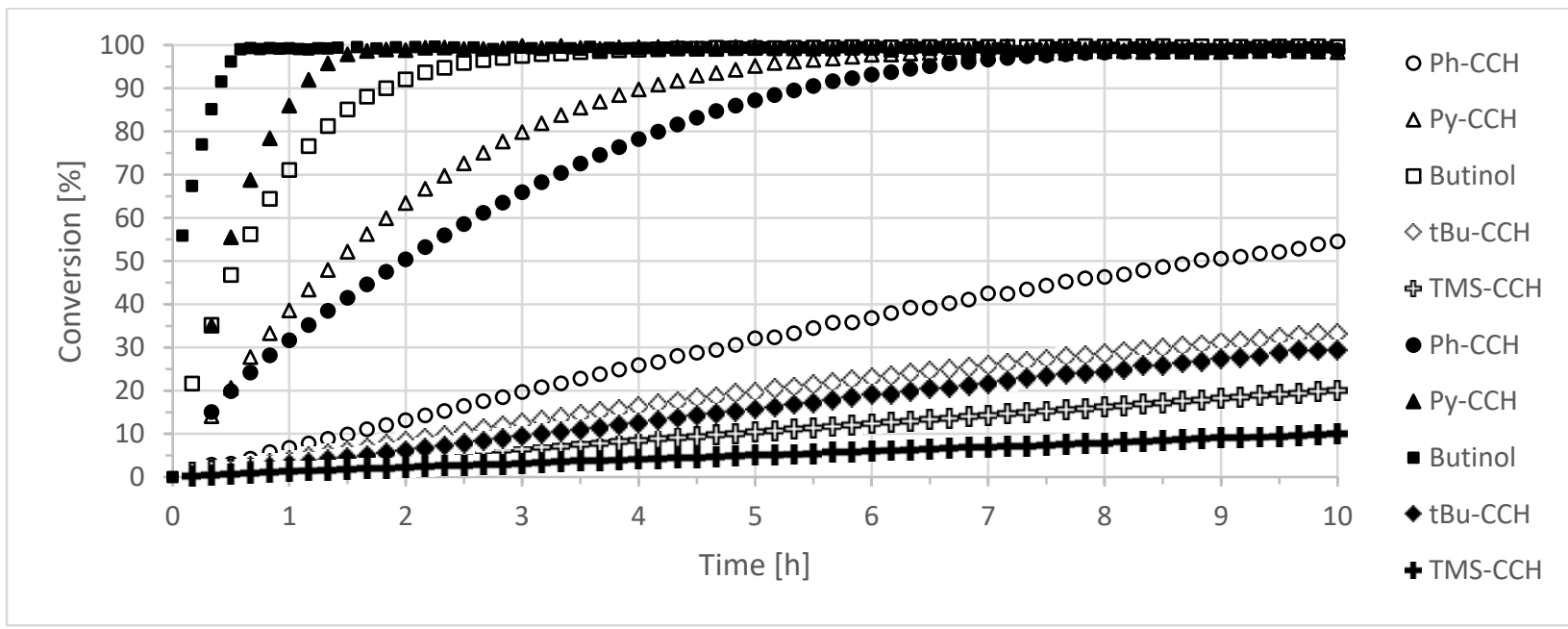

Processing of the spectra was done using MestReNova 10.0.2-15465 by Mestrelab Research. Stacked spectra were evaluated using the Data Analysis tool. The integrated peaks were normalized as a function of the internal standard and further processing was done with Microsoft Excel.

Within the framework of these catalytic investigations, blank tests were performed for crosschecking to ensure that the determined conversion rates did not originate from possible traces of neat $\mathrm{Cu}(\mathrm{I}) \mathrm{Br}$. Therefore, under the same conditions mentioned above, $1 \mathrm{~mol} \%$ of $\mathrm{CuBr}\left(\mathrm{SMe}_{2}\right)$ was added instead of complex Fehler! Verweisquelle konnte nicht gefunden werden.b/Fehler! Verweisquelle konnte nicht gefunden werden.b and the resulting reaction mixture was monitored via ${ }^{1} \mathrm{H}$ NMR measurements periodic intervals. The conversion times to the formation of the 
triazoles P1-3 were partly significantly longer (P1: 40 h, 96\%; P2: 16 h, 100\%; P3: 40 h, 67\%), whereas the formation of P4-5 showed similar conversion times (P4: 40 h; 96\%; P5: 40 h; 82\%).

\begin{tabular}{|c|c|c|c|c|c|c|c|c|c|c|c|}
\hline \multirow[b]{2}{*}{$\begin{array}{c}\text { Time } \\
{[\mathrm{h}]}\end{array}$} & \multirow[b]{2}{*}{$\begin{array}{c}\text { 2b } \\
\text { PhCCH }\end{array}$} & \multicolumn{10}{|c|}{ Conversion (Integrated values; $\approx 0-100.000$ ) } \\
\hline & & $\begin{array}{c}\text { 2b } \\
\text { PyCCH }\end{array}$ & $\begin{array}{c}2 b \\
\text { Butinol }\end{array}$ & $\begin{array}{c}2 \mathrm{~b} \\
t \mathrm{BuCCH}\end{array}$ & $\begin{array}{c}2 \mathbf{b} \\
\mathrm{TMSCCH}\end{array}$ & $\begin{array}{c}3 b \\
\text { PhCCH }\end{array}$ & $\begin{array}{c}3 b \\
\text { PyCCH }\end{array}$ & $\begin{array}{c}\text { Time } \\
\text { [h] }\end{array}$ & $\begin{array}{c}\mathbf{3 b} \\
\text { Butinol }\end{array}$ & $\begin{array}{c}3 b \\
t \mathrm{BuCCH}\end{array}$ & $\begin{array}{c}\text { 3b } \\
\mathrm{TMSCCH}\end{array}$ \\
\hline 0.167 & 1.451 & 0.641 & 1.626 & 0.120 & 1.075 & 0.327 & 0.539 & 0.083 & 55.853 & 0.123 & 0.185 \\
\hline 0.333 & 2.833 & 14.059 & 35.238 & 1.150 & 1.497 & 15.048 & 35.035 & 0.167 & 67.359 & 513 & .509 \\
\hline 0.5 & 3.087 & 20.600 & 46.761 & 1.686 & 1.816 & 19.859 & 55.498 & .250 & 76.988 & .218 & 0.733 \\
\hline 0.667 & 4.260 & 27.749 & 56.201 & 2.366 & 2.124 & 24.195 & 68.760 & 0.333 & 85.144 & 1.790 & 0.933 \\
\hline 0.833 & 5.774 & 33.267 & 64.405 & 3.093 & 2.445 & 28.166 & 78.410 & 0.417 & 91.523 & 2.363 & 1.115 \\
\hline 1 & 6.752 & 38.578 & 71.068 & 3.852 & 2.761 & 31.642 & 85.994 & 0.500 & 96.190 & 2.885 & 1.277 \\
\hline 1.167 & 7.781 & 43.416 & 76.588 & 4.585 & 3.068 & 35.184 & 91.974 & .583 & 99.025 & 357 & 1.451 \\
\hline 1.333 & 8.824 & 47.959 & 81.234 & 5.310 & 3.369 & 38.487 & 95.785 & .667 & 99.306 & .928 & 1.613 \\
\hline 1.5 & 9.845 & 52.147 & 85.084 & 6.043 & 3.711 & 41.501 & 97.864 & .750 & 99.082 & 457 & 734 \\
\hline 1.667 & 11.078 & 56.236 & 88.087 & 6.766 & 4.005 & 44.607 & 98.666 & 833 & 99.312 & 972 & .936 \\
\hline 1.833 & 12.044 & 59.931 & 90.017 & 7.543 & 4.330 & 47.521 & 98.983 & .917 & 99.148 & .578 & .057 \\
\hline 2 & 13.174 & 63.423 & 92.054 & 8.274 & 4.645 & 50.420 & 98.829 & 1.000 & 99.263 & .135 & 2.237 \\
\hline 2.167 & 14.262 & 66.715 & 93.689 & 8.986 & 4.944 & 53.245 & 99.392 & 1.083 & 99.070 & .704 & 2.409 \\
\hline 2.333 & 15.306 & 69.759 & 94.725 & 9.671 & 5.267 & 55.968 & 99.439 & 1.167 & 98.974 & 216 & 2.587 \\
\hline 2.5 & 16.446 & 72.608 & 95.853 & 10.461 & 5.592 & & 26 & 50 & & & 45 \\
\hline 2.667 & 17.550 & 75.101 & 96.500 & 11.098 & 5.909 & 61.179 & .110 & 333 & 99 & 372 & 2.866 \\
\hline 2.833 & 18.505 & 77.692 & 96.998 & 11.795 & 6.221 & 63.541 & 9.229 & 417 & 99.390 & 859 & 2.946 \\
\hline 3 & 19.707 & 79.852 & 97.478 & 12.417 & 6.542 & 65.960 & 9.808 & 583 & 99.519 & .452 & 168 \\
\hline 3.167 & 20.802 & 81.899 & 97.881 & 13.131 & 6.875 & 68.254 & 99.301 & 1.750 & 99.198 & 10.007 & 3.327 \\
\hline 3.333 & 21.686 & 83.778 & 98.035 & 13.750 & 7.178 & 70.393 & 99.824 & 1.917 & 99.501 & 10.415 & 3.474 \\
\hline 3.5 & 22.790 & 85.528 & 98.349 & 14.439 & 7.507 & 72.552 & 99.351 & 2.083 & 99.521 & 10.925 & 3.714 \\
\hline 3.667 & 23.845 & 86. & 98.598 & 15.096 & 7.837 & 74. & 31 & 250 & & & 3.856 \\
\hline 3.833 & 24.875 & 88.436 & 98.783 & 15.619 & 8.160 & 76.393 & 99.468 & 2.417 & 99.429 & 12.023 & 3.921 \\
\hline 4 & 25.909 & 89.726 & 98.930 & 16.296 & 8 & 78.227 & 99.541 & 2.583 & 99.442 & 12.370 & 4.120 \\
\hline 4.167 & 26.609 & 90.872 & 99.007 & 16.885 & 8.793 & 79.959 & 99.570 & .750 & 99.078 & \begin{tabular}{|l|}
13.078 \\
\end{tabular} & 4.302 \\
\hline 4.333 & 28.036 & 91.738 & 99.181 & 17.487 & 9.117 & 81.611 & 99.490 & 2.917 & 99.485 & 13.703 & 4.390 \\
\hline 4.5 & 28.804 & 92.950 & 99.140 & 18.168 & 9.421 & 83.207 & 99.250 & 3.083 & 99.264 & \begin{tabular}{|l}
14.276 \\
\end{tabular} & 4.520 \\
\hline 4.667 & 29.421 & 93.519 & 99.383 & 18.619 & 9.731 & 84.715 & 99.105 & 3.250 & 99.317 & 14.604 & 4.725 \\
\hline 4.833 & 30.604 & 94.299 & 99.272 & 19.277 & 10.064 & 85.988 & 99.570 & 3.417 & 99.226 & 15.105 & 4.876 \\
\hline 5 & 32.142 & 95.161 & 99.342 & 19.674 & 10.412 & 87211 & 99.660 & 3.583 & 99.553 & 96 & 5.069 \\
\hline 5.167 & 32.363 & 95.852 & 99.276 & 20.407 & 10.700 & 88.451 & 99.280 & 3.750 & 99.318 & 16.158 & 5.153 \\
\hline 5.333 & 33.269 & 96.198 & 99.380 & 20.842 & 11.078 & 89.523 & 99.251 & 3.917 & 99.252 & 16.783 & 5.261 \\
\hline 5.5 & 34.497 & 96.540 & 99.413 & 21.362 & 11.352 & 90.508 & 99.212 & 4.083 & 99.292 & 17.062 & 5.403 \\
\hline 5.667 & 35.727 & 96.868 & 99.500 & 21.891 & 11.679 & 91.619 & 99.192 & 4.250 & 99.396 & 17.710 & 5.772 \\
\hline 5.833 & 35.805 & 97.285 & 99.528 & 22.386 & 11.989 & 92.328 & 99.397 & 4.417 & 99.494 & 18.543 & 5.779 \\
\hline 6 & 36.833 & 97.742 & 99.526 & 23.000 & 12.333 & 93.168 & 99.399 & 4.583 & 99.484 & 19.095 & 5.922 \\
\hline 6.167 & 37.975 & 97.826 & 99.457 & 23.427 & 12.672 & 93.724 & 98.905 & 4.750 & 99.590 & \begin{tabular}{|l|}
19.153 \\
\end{tabular} & 6.108 \\
\hline 6.333 & 39.233 & 98.058 & 99.632 & 24.065 & 12.980 & 94.535 & 99.671 & 4.917 & 99.407 & 19.924 & 6.261 \\
\hline 6.5 & 39.154 & 98.19 & 99.578 & 24.461 & 13.342 & 95.130 & 99.290 & 5.083 & 99.412 & 20.411 & 6.533 \\
\hline 6.667 & 40.220 & 98.001 & 99.643 & 24.913 & 13.623 & 95.762 & 99.583 & 5.250 & 99.543 & 20.603 & 6.728 \\
\hline 6.833 & 41.087 & 98.390 & 99.709 & 25.405 & 13.948 & 96.072 & 99.070 & 5.417 & 99.426 & 21.044 & 6.847 \\
\hline 7 & 42.496 & 97.885 & 99.607 & 25.793 & 14.269 & 96.637 & 99.227 & 5.583 & 99.390 & 21.582 & 6.885 \\
\hline 7.167 & 42.424 & 98.234 & 99.628 & 26.304 & 14.617 & 97.043 & 99.699 & 5.750 & 99.492 & 22.301 & 7.113 \\
\hline 7.333 & 43.449 & 98.279 & 99.637 & 26.695 & 14.917 & 97.455 & 99.169 & 5.917 & 99.474 & \begin{tabular}{|l|}
22.888 \\
\end{tabular} & 7.172 \\
\hline 7.5 & 44.320 & 98.122 & 99.605 & 27.299 & 15.268 & 97.640 & 99.165 & 6.083 & 99.515 & 23.332 & 7.407 \\
\hline 7.667 & 45.247 & 98.194 & 99.652 & 27.813 & 15.563 & 97.844 & 99.556 & 6.250 & 99.372 & 23.831 & 7.531 \\
\hline
\end{tabular}




\begin{tabular}{|c|c|c|c|c|c|c|c|c|c|c|c|}
\hline 7.833 & 46.013 & 98.497 & 99.775 & 28.011 & 15.888 & 98.180 & 98.968 & 6.417 & 99.457 & 24.034 & 7.789 \\
\hline 8 & 46.318 & 98.578 & 99.687 & 28.506 & 16.197 & 98.284 & 99.398 & 6.583 & 99.510 & 24.207 & 7.847 \\
\hline 8.167 & 46.943 & 98.588 & 99.693 & 29.050 & 16.526 & 98.423 & 98.773 & 6.750 & 99.135 & 24.797 & 8.141 \\
\hline 8.333 & 47.829 & 98.430 & 99.717 & 29.460 & 16.845 & 98.602 & 99.532 & 6.917 & 99.457 & 25.743 & 8.306 \\
\hline 8.5 & 48.624 & 98.477 & 99.752 & 29.896 & 17.229 & 98.554 & 99.437 & 7.083 & 99.258 & 25.751 & 8.499 \\
\hline 8.667 & 49.270 & 98.487 & 99.594 & 30.234 & 17.515 & 98.636 & 99.041 & 7.250 & 98.562 & 26.402 & 8.694 \\
\hline 8.833 & 50.209 & 98.357 & 99.689 & 30.633 & 17.817 & 98.719 & 98.972 & 7.417 & 99.171 & 26.707 & 8.820 \\
\hline 9 & 50.543 & 98.427 & 99.684 & 31.092 & 18.208 & 98.537 & 99.526 & 7.583 & 99.412 & 27.476 & 9.075 \\
\hline 9.167 & 50.996 & 98.596 & 99.597 & 31.346 & 18.454 & 98.654 & 99.354 & 7.750 & 99.370 & 27.662 & 9.118 \\
\hline 9.333 & 51.759 & 98.580 & 99.570 & 31.857 & 18.857 & 98.688 & 99.318 & 7.917 & 99.509 & 27.920 & 9.230 \\
\hline 9.5 & 52.127 & 98.838 & 99.672 & 32.259 & 19.116 & 98.705 & 99.717 & 8.083 & 99.325 & 28.715 & 9.405 \\
\hline 9.667 & 52.851 & 98.556 & 99.691 & 32.714 & 19.461 & 98.696 & 99.213 & 8.250 & 99.393 & 29.464 & 9.641 \\
\hline 9.833 & 53.898 & 98.482 & 99.688 & 33.160 & 19.786 & 98.684 & 99.294 & 8.417 & 99.457 & 29.281 & 9.794 \\
\hline 10 & 54.548 & 98.356 & 99.616 & 33.248 & 20.101 & 98.767 & 100.187 & 8.583 & 99.324 & 29.339 & 9.988 \\
\hline & & & & & & & & 8.750 & 99.190 & & \\
\hline & & & & & & & & 8.917 & 99.065 & & \\
\hline & & & & & & & & 9.083 & 99.186 & & \\
\hline & & & & & & & & 9.250 & 99.484 & & \\
\hline & & & & & & & & 9.417 & 99.504 & & \\
\hline & & & & & & & & 9.583 & 99.558 & & \\
\hline & & & & & & & & 9.750 & 99.441 & & \\
\hline & & & & & & & & 9.917 & 99.486 & & \\
\hline & & & & & & & & 10.083 & 99.422 & & \\
\hline
\end{tabular}




\section{KAT_2b_Ph-CCH}

Alkyne: Ph-CCH (1.00 eq); Azide: benzyl azide (1.00 eq); Internal Standard: adamantane; dcm-d2; 10 min steps.

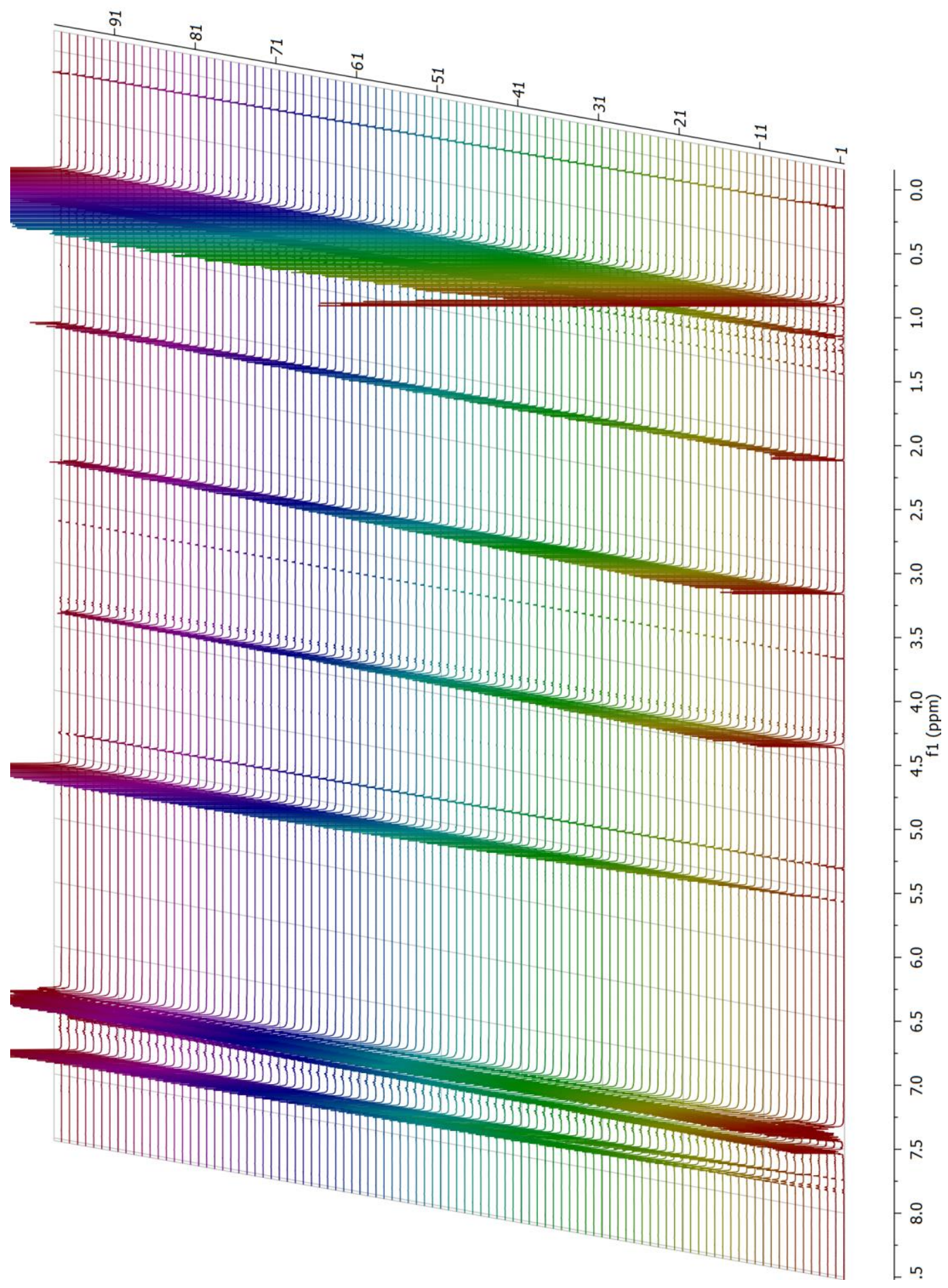




\section{KAT_2b_Py-CCH}

Alkyne: Py-CCH (1.00 eq); Azide: benzyl azide (1.00 eq); Internal Standard: adamantane; dcm-d2;

10 min steps.

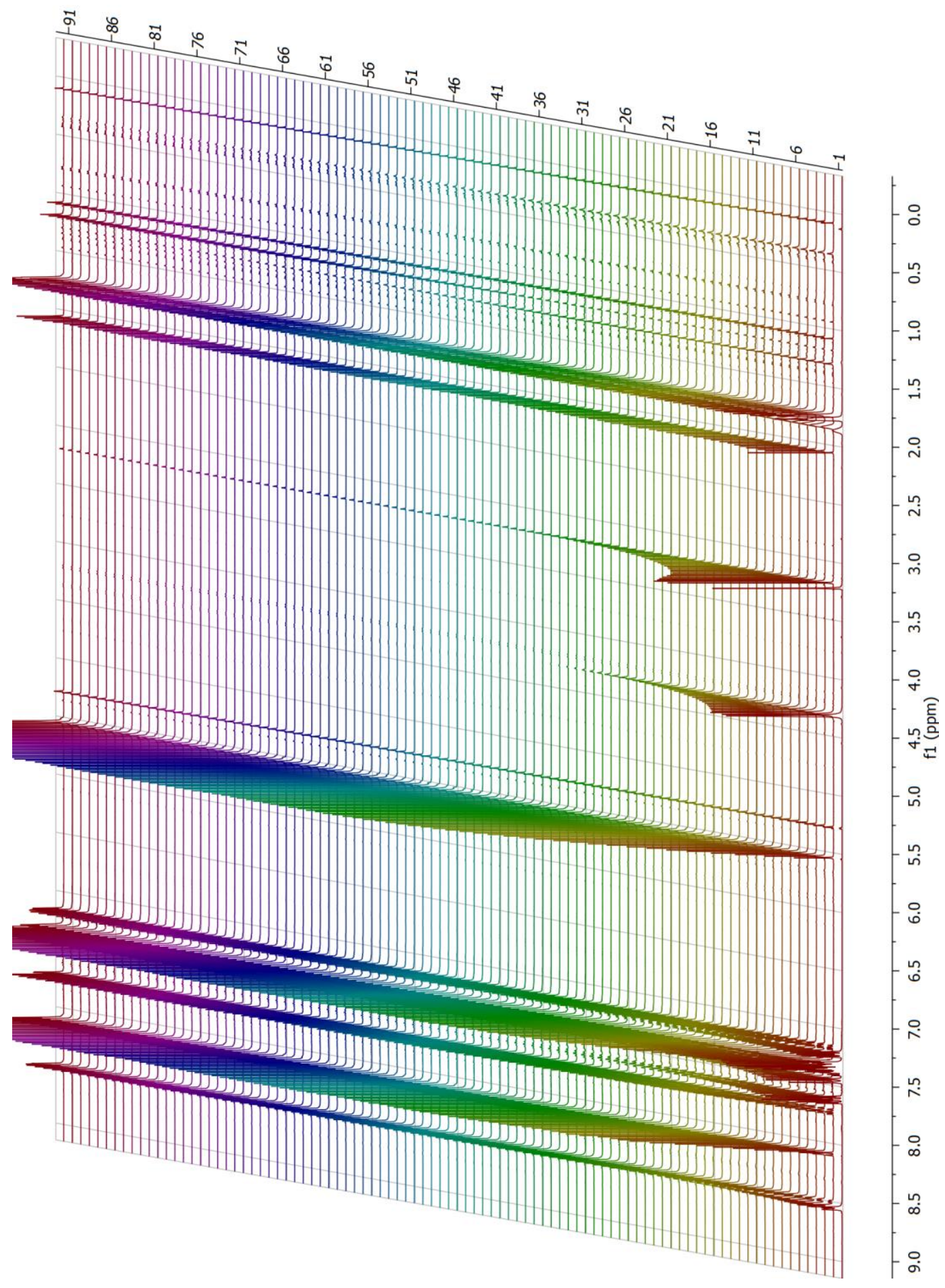




\section{KAT_2b_Butinol}

Alkyne: Butinol (1.00 eq); Azide: benzyl azide (1.00 eq); Internal Standard: adamantane; dcm-d2; 10 min steps.

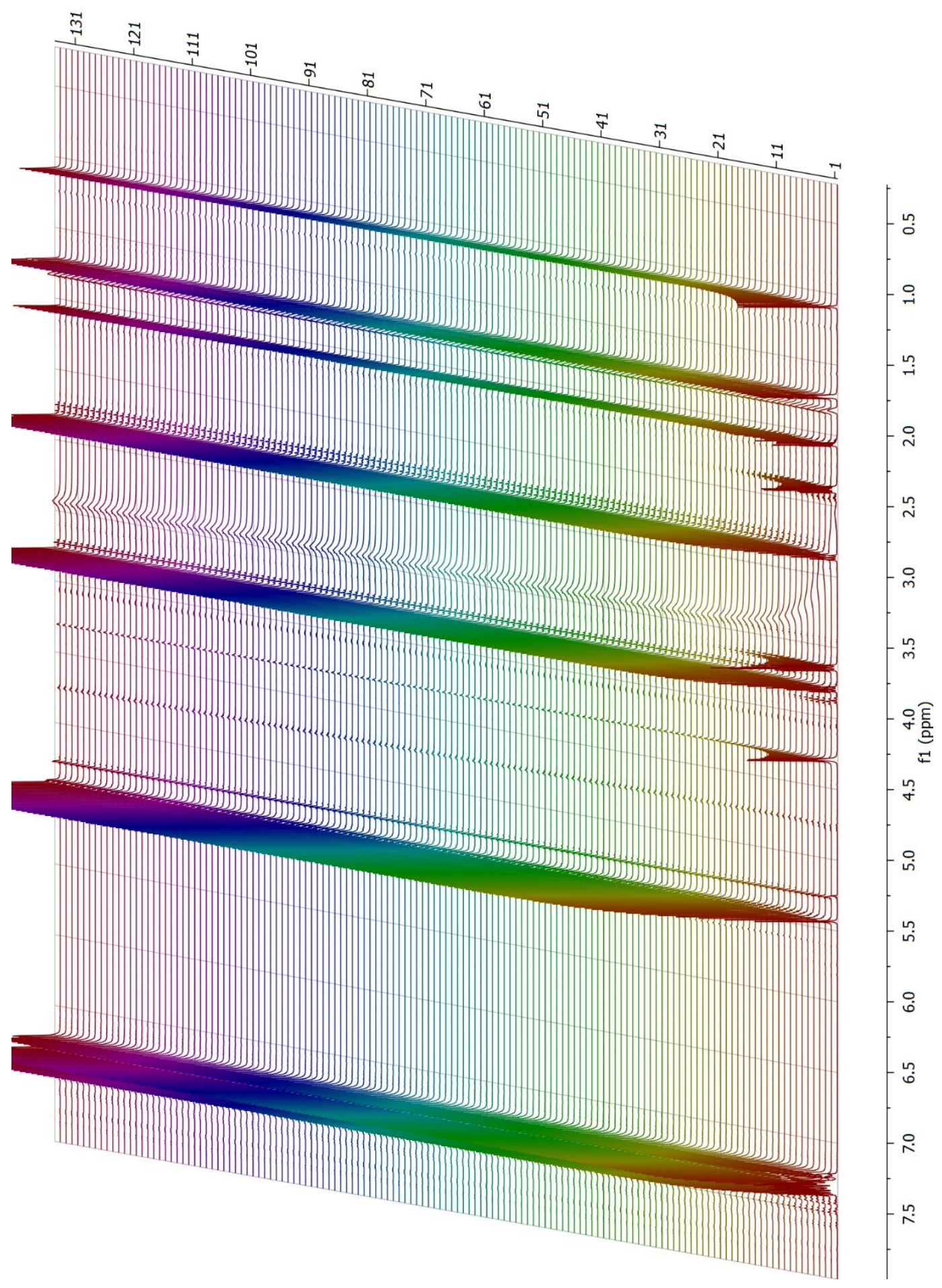




\section{KAT_2b_tBu-CCH}

Alkyne: $\mathbf{t B u}-\mathbf{C C H}$ (1.00 eq); Azide: benzyl azide (1.00 eq); Internal Standard: adamantane; dcm-d2; 10 min steps.

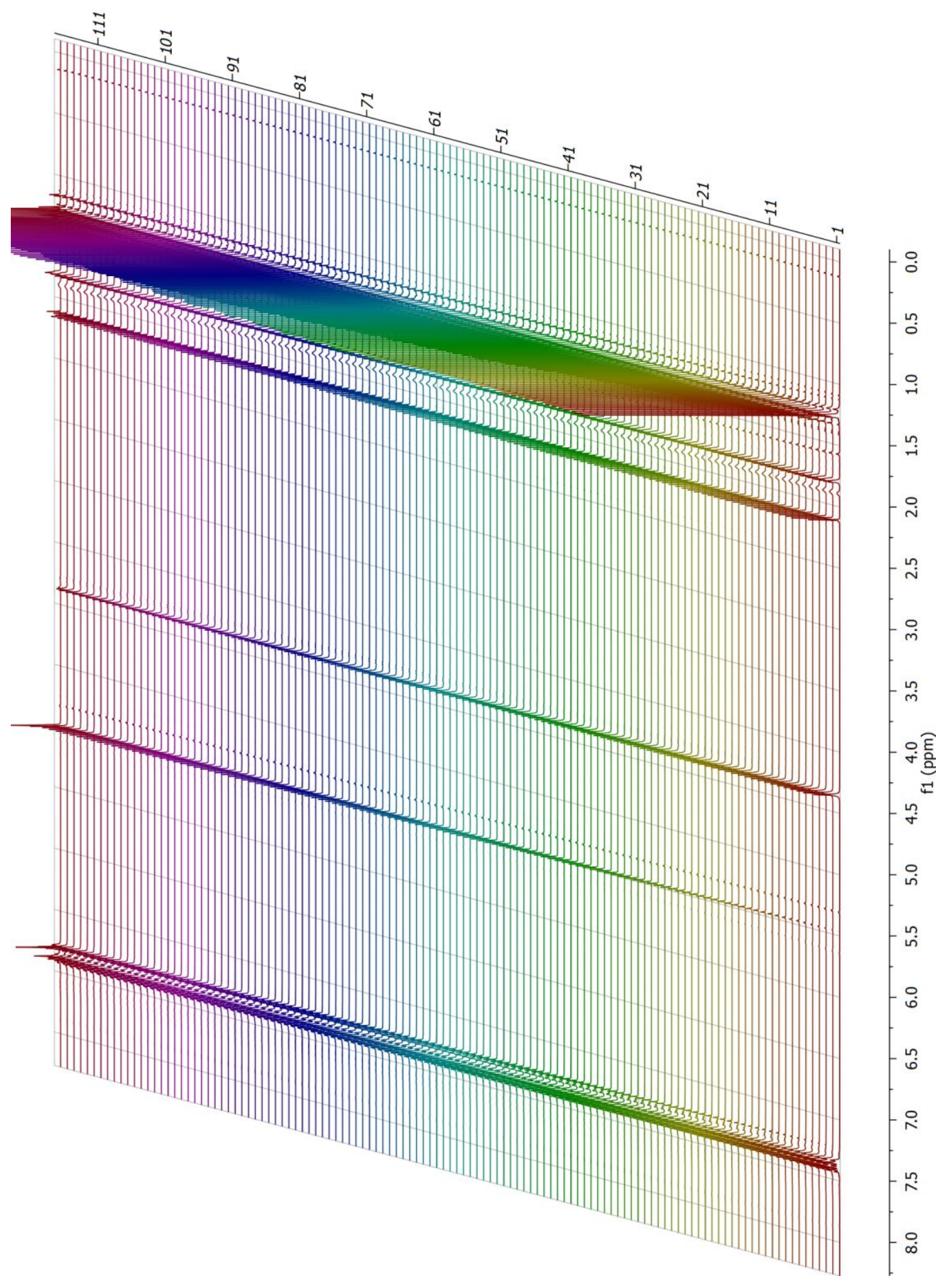




\section{KAT_2b_TMS-CCH}

Alkyne: TMS-CCH (1.00 eq); Azide: benzyl azide (1.00 eq); Internal Standard: adamantane; dcmd2; 10 min steps.

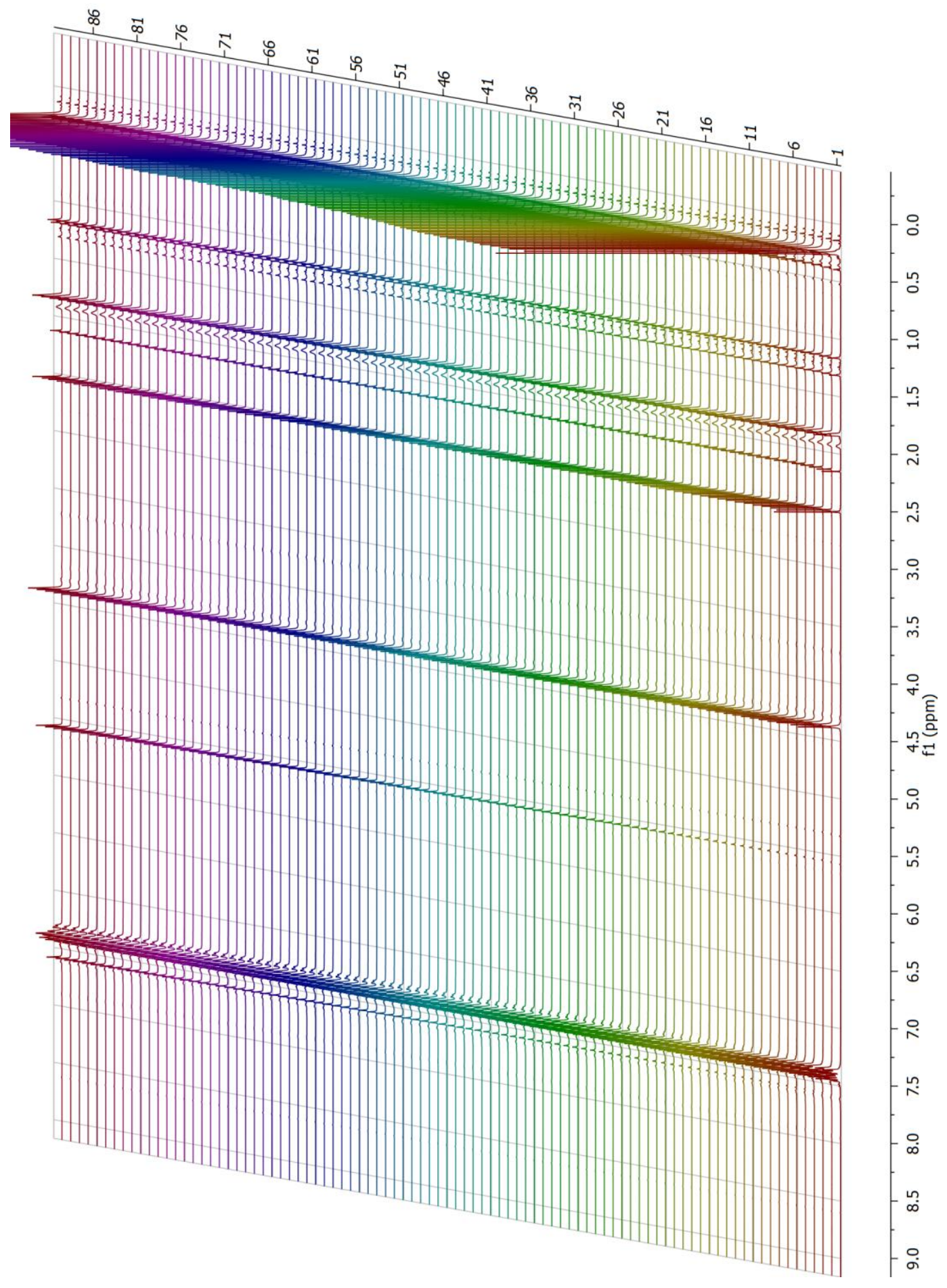




\section{KAT_3b_Ph-CCH}

Alkyne: Ph-CCH (1.00 eq); Azide: benzyl azide (1.00 eq); Internal Standard: adamantane; dcm-d2; 10 min steps.

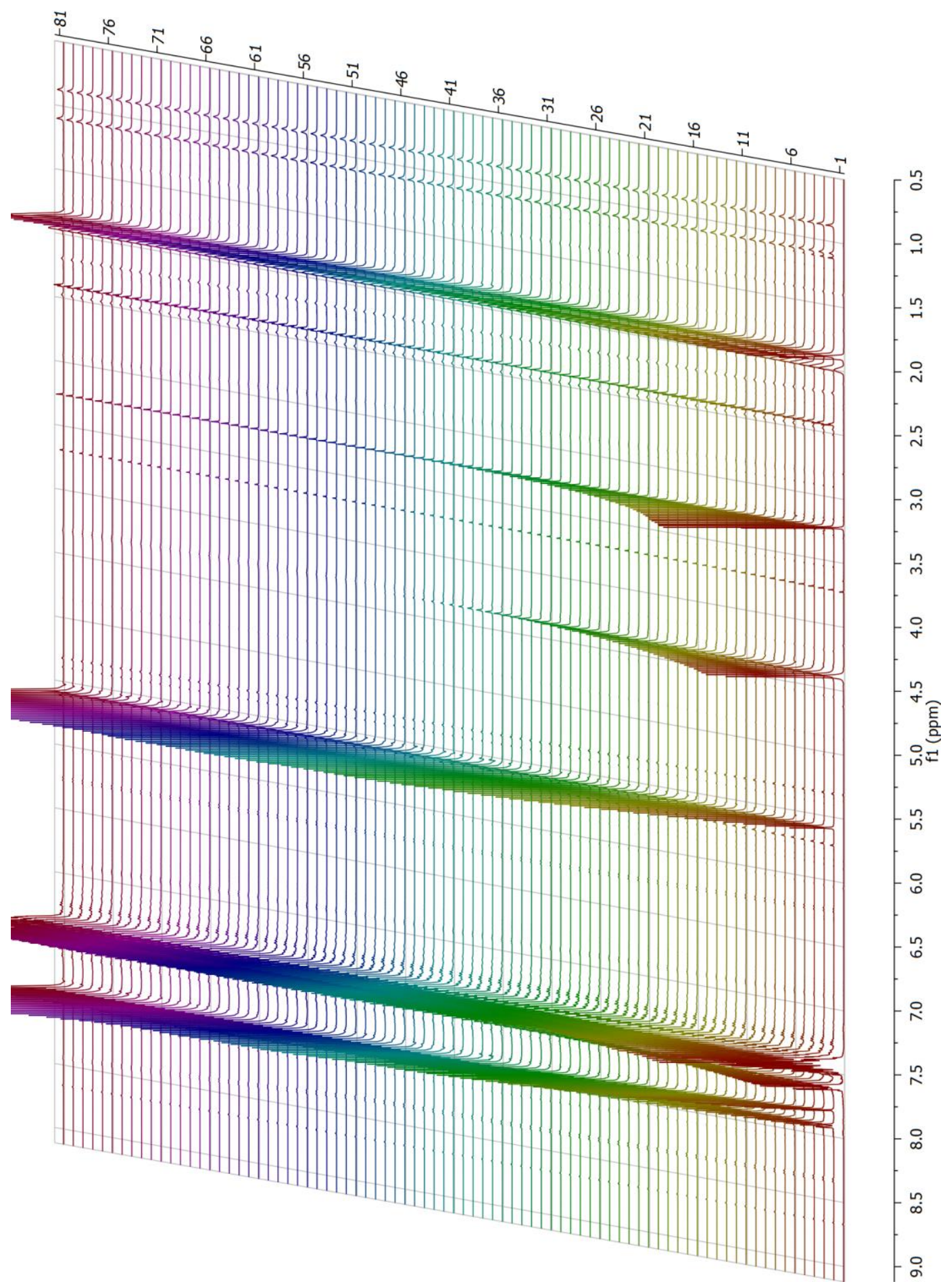




\section{KAT_3b_Py-CCH}

Alkyne: Py-CCH (1.00 eq); Azide: benzyl azide (1.00 eq); Internal Standard: adamantane; dcm-d2; 10 min steps.

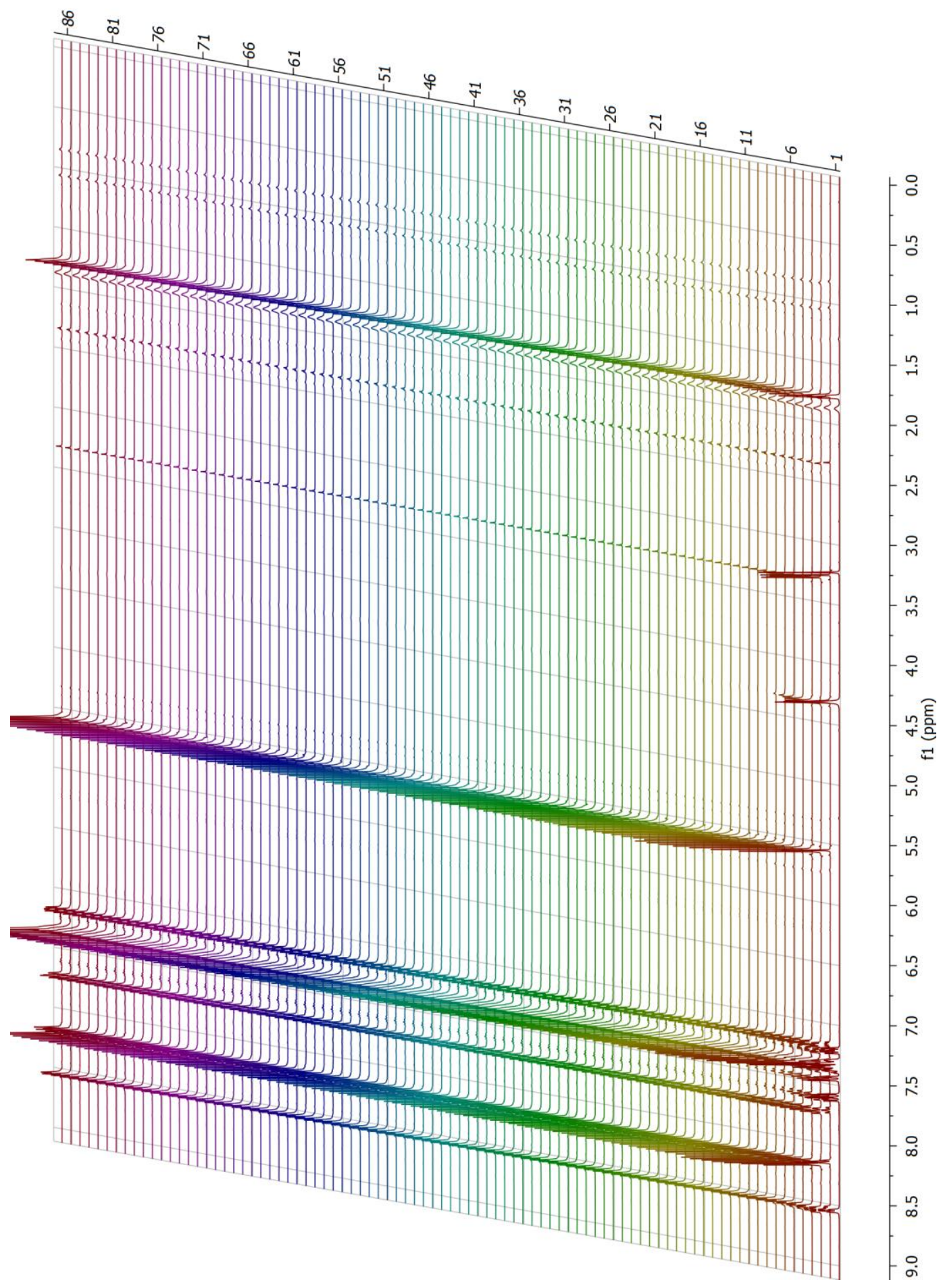




\section{KAT_3b_Butinol}

Alkyne: Butinol (1.00 eq); Azide: benzyl azide (1.00 eq); Internal Standard: adamantane; dcm-d2;

$5 / 10$ min steps.

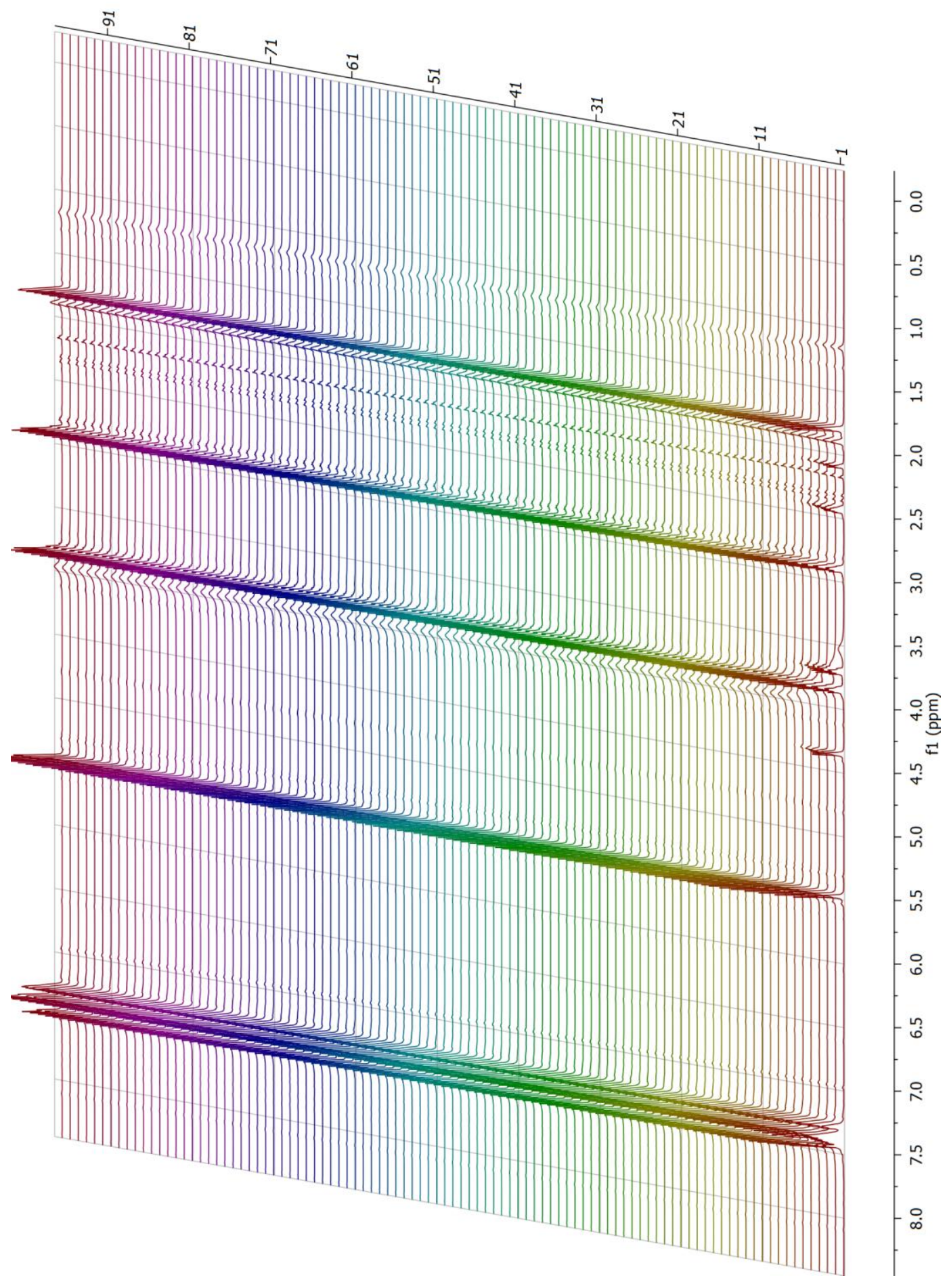




\section{KAT_3b_tBu-CCH}

Alkyne: $\mathbf{t B u}-\mathbf{C C H}$ (1.00 eq); Azide: benzyl azide (1.00 eq); Internal Standard: adamantane; dcm-d2; 10 min steps.

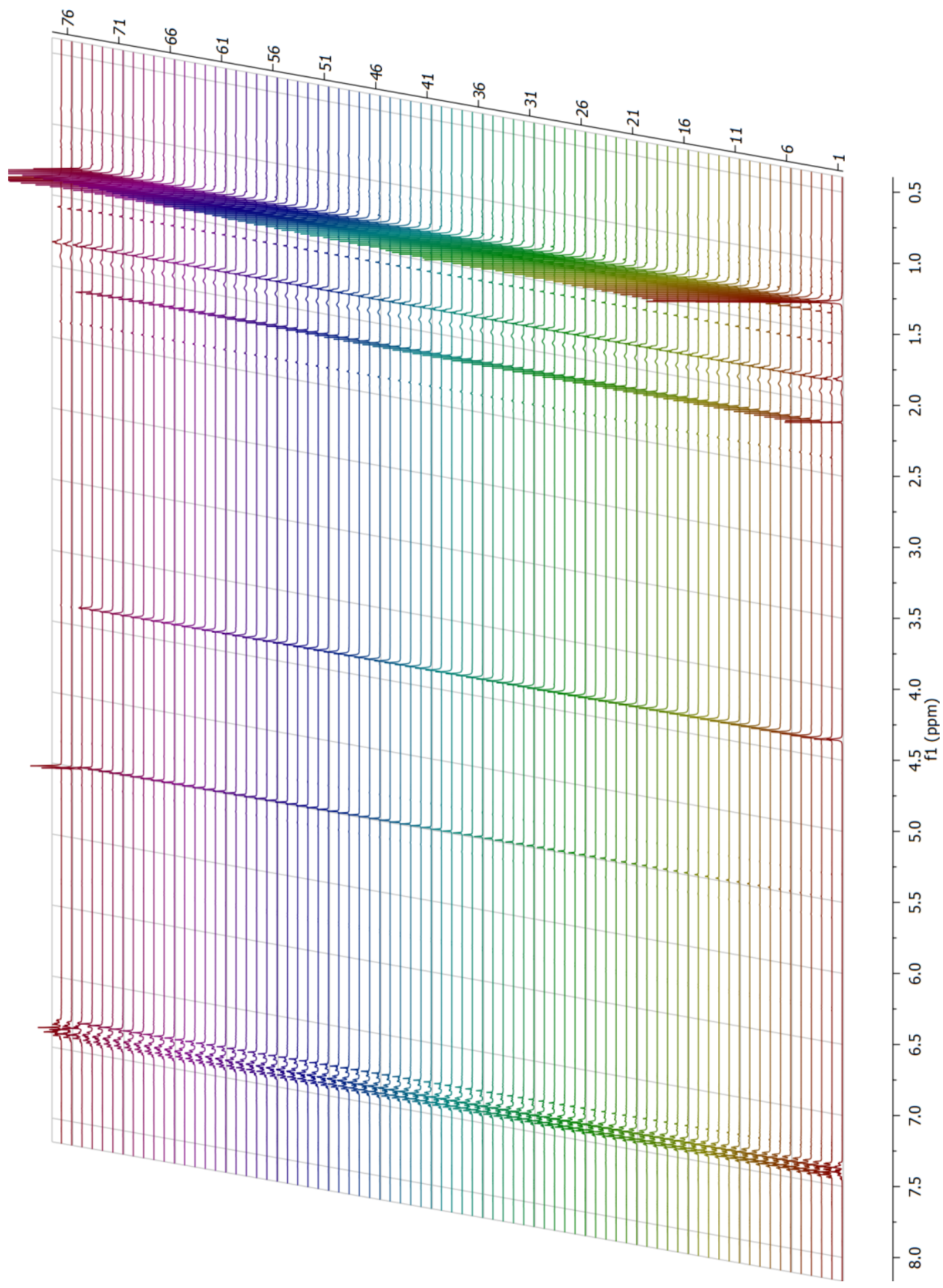




\section{KAT_3b_TMS-CCH}

Alkyne: TMS-CCH (1.00 eq); Azide: benzyl azide (1.00 eq); Internal Standard: adamantane; dcmd2; 10 min steps.

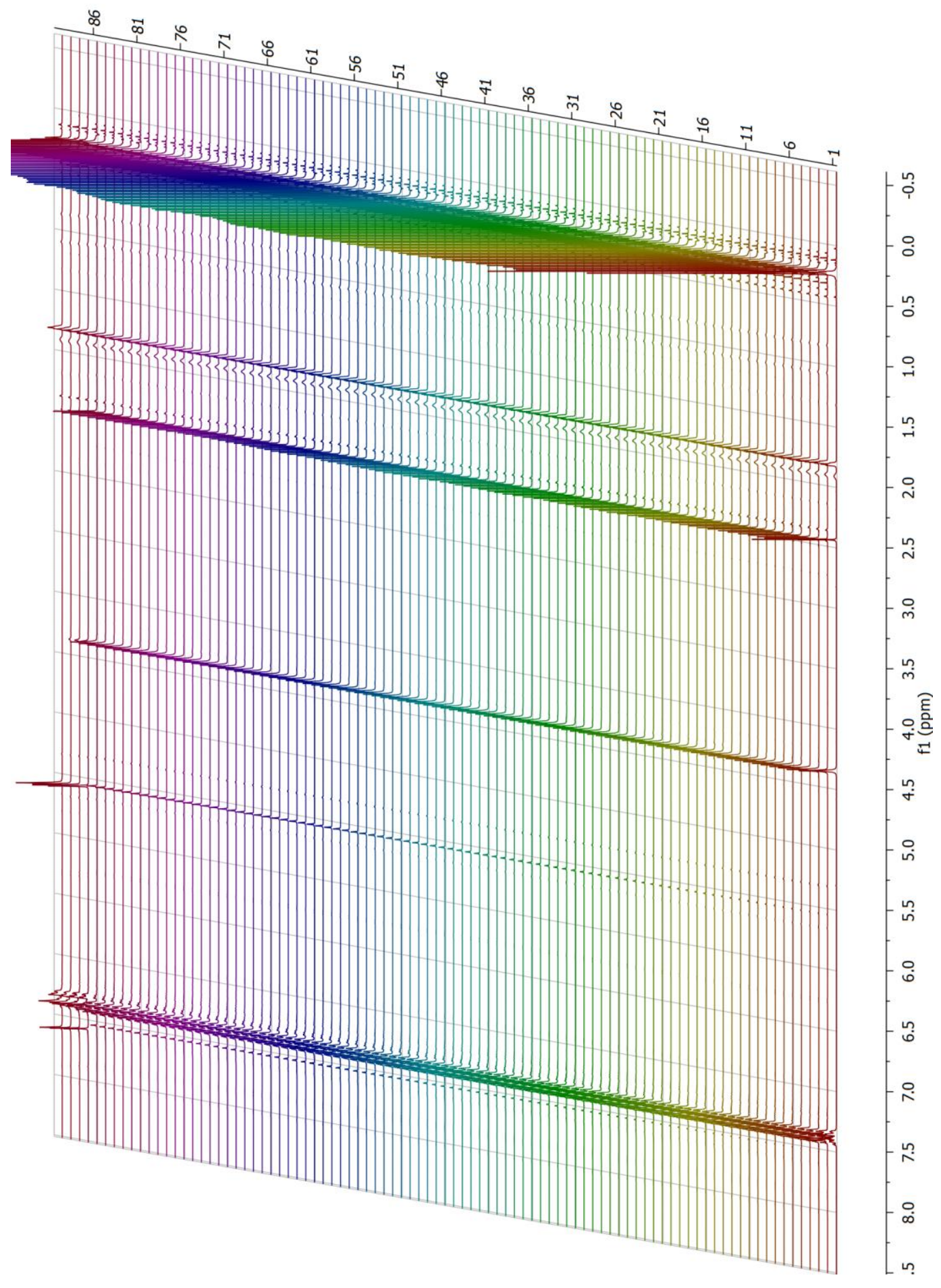




\section{S4. References}

[1] Georg-August-University, "Virtuelles Labor I", can be found under http://www.stalke.chemie.unigoettingen.de/virtuelles_labor/advanced/13_de.html, 2014.

[2] C.-W. So, H. W. Roesky, J. Magull, R. B. Oswald, Angew. Chem., Int. Ed. 2006, 45, 3948.

[3] S. S. Sen, H. W. Roesky, D. Stern, J. Henn, D. Stalke, J. Am. Chem. Soc. 2010, 132, 1123.

[4] J. J. Allen, C. E. Hamilton, A. R. Barron, J. Chem. Crystallogr. 2009, 39, 573.

[5] S. Ngubane, K. M. Kadish, J. L. Bear, E. van Caemelbecke, A. Thuriere, K. P. Ramirez, Dalton Trans. 2013, 42, 3571.

[6] S. Bachmann, B. Gernert, D. Stalke, Chem. Commun. 2016, 52, 12861.

[7] R. Neufeld, D. Stalke, Chem. Sci. 2015, 6, 3354.

[8] A.-K. Kreyenschmidt, S. Bachmann, T. Niklas, D. Stalke, ChemistrySelect 2017, 2, 6957. 